\title{
THE GEOMETRY OF GROUPS CONTAINING ALMOST NORMAL SUBGROUPS
}

\author{
ALEXANDER MARGOLIS
}

\begin{abstract}
A subgroup $H \leq G$ is said to be almost normal if every conjugate of $H$ is commensurable to $H$. If $H$ is almost normal, there is a well-defined quotient space $G / H$. We show that if a group $G$ has type $F_{n+1}$ and contains an almost normal coarse $P D_{n}$ subgroup $H$ with $e(G / H)=\infty$, then whenever $G^{\prime}$ is quasi-isometric to $G$ it contains an almost normal subgroup $H^{\prime}$ that is quasi-isometric to $H$. Moreover, the quotient spaces $G / H$ and $G^{\prime} / H^{\prime}$ are quasi-isometric. This generalises a theorem of Mosher-Sageev-Whyte, who prove the case in which $G / H$ is quasi-isometric to a finite valence bushy tree. Using work of Mosher, we generalise a result of Farb-Mosher to show that for many surface group extensions $\Gamma_{L}$, any group quasi-isometric to $\Gamma_{L}$ is virtually isomorphic to $\Gamma_{L}$. We also prove quasi-isometric rigidity for the class of finitely presented $\mathbb{Z}$-by- $(\infty$ ended $)$ groups.
\end{abstract}

\section{INTRODUCTION}

A central idea in geometric group theory is that a finitely generated group equipped with the word metric is a geometric object in its own right. This metric is well-defined up to quasi-isometry. A typical question is the following: given a class $\mathcal{C}$ of finitely generated groups, is it true that any finitely generated group quasi-isometric to an element of $\mathcal{C}$ is also in $\mathcal{C}$ ? Positive answers to this question occur surprisingly often, and this phenomenon is called quasi-isometric rigidity. A related problem is quasi-isometric classification: when are groups in $\mathcal{C}$ quasi-isometric? These questions comprise Gromov's program of studying finitely generated groups up to quasi-isometry.

One approach to these questions is to show that if a group $G$ can be decomposed into "smaller" or "simpler" groups, then any group quasi-isometric to $G$ also decomposes in a similar way. The aim of this article is to investigate instances in which the following question and generalisations of it are true:

Question 1.1. Suppose a finitely generated group $G$ contains an infinite normal subgroup $H$. If $G^{\prime}$ is a finitely generated group quasi-isometric to $G$, does it contain a normal subgroup $H^{\prime}$ such that $H$ is quasi-isometric to $H^{\prime}$ and $G / H$ is quasi-isometric to $G^{\prime} / H^{\prime}$ ?

Although this question is false in full generality, we can nonetheless give several instances for which there is a positive answer. These are applications of Theorem 1.4, the main result of this article. We say that a group is $\mathbb{Z}-b y-$ $(\infty$ ended) if it has an infinite cyclic normal subgroup such that the quotient is infinite ended. Our first result says that the class of finitely presented $\mathbb{Z}$-by-( $\infty$ ended) groups is quasi-isometrically rigid:

This research was supported by the Israel Science Foundation (grant No. 1026/15). 
Theorem 5.10. Let $G$ be a finitely presented $\mathbb{Z}-b y-(\infty$ ended $)$ group. If $G^{\prime}$ is any finitely generated group quasi-isometric to $G$, it is also $\mathbb{Z}-b y-(\infty$ ended). Moreover, any quasi-isometry $f: G \rightarrow G^{\prime}$ induces a quasi-isometry $G / \mathbb{Z} \rightarrow G^{\prime} / \mathbb{Z}$ between quotient groups.

A group $G$ is of type $F_{n}$ if it has a $K(G, 1)$ with finite $n$-skeleton. We say that $G$ is $\mathbb{Z}^{n}-b y-(\infty$ ended) with almost injective quotient (AIQ) if $G$ has a normal subgroup $H \cong \mathbb{Z}^{n}$ such that the quotient is infinite ended and the natural homomorphism $G / H \rightarrow \operatorname{Aut}(H) \cong G L(n, \mathbb{Z})$ has finite kernel. We show that this class of groups is quasi-isometrically rigid:

Theorem 5.16. Let $G$ be a group of type $F_{n+1}$ that is $\mathbb{Z}^{n}$-by-( $\infty$ ended) with AIQ. If $G^{\prime}$ is any finitely generated group quasi-isometric to $G$, then it is also $\mathbb{Z}^{n}-$ by- $(\infty$ ended $)$ with $A I Q$. Moreover, any quasi-isometry $f$ : $G \rightarrow G^{\prime}$ induces a quasi-isometry $G / \mathbb{Z}^{n} \rightarrow G^{\prime} / \mathbb{Z}^{n}$ between the infinite ended quotients.

However, Theorem 5.16] does not hold if we relax the AIQ hypothesis. For instance, in Example 5.11 we describe a group quasi-isometric to $\mathbb{Z}^{2} \times F_{2}$ that does not (virtually) contain a free abelian normal subgroup of rank 2. Similar examples are also considered in work of Leary-Minasyan [LM19. This resolves a question of [FLS15, Section 12.2].

Using work of Mosher [Mos03a], we can also prove a much stronger form of quasi-isometric rigidity for certain surface group extensions. The DehnNielsen-Baer theorem says that $\operatorname{Out}\left(\pi_{1}(S)\right) \cong \operatorname{MCG}(S)$, where $\operatorname{MCG}(S)$ is the extended mapping class group of a closed hyperbolic surface $S$. Given a subgroup $L \leq \operatorname{MCG}(S)$, let $\Gamma_{L}$ be the associated surface group extension $1 \rightarrow \pi_{1}(S) \rightarrow \Gamma_{L} \rightarrow L \rightarrow 1$. We say that $L$ is irreducible if it doesn't preserve a finite collection of disjoint simple closed curves.

Theorem A.19. Suppose $L$ is an irreducible subgroup of $\operatorname{MCG}(S)$ that is of type $F_{3}$ and has infinitely many ends. Let $\Gamma_{L}$ be the associated surface group extension. If $G$ is any finitely generated group quasi-isometric to $\Gamma_{L}$, then there is a finite normal subgroup $N \triangleleft G$ such that $G / N$ is abstractly commensurable to $\Gamma_{L}$, i.e. $\Gamma_{L}$ and $G / N$ have isomorphic finite index subgroups.

This generalises a result of Farb-Mosher [FM02a], who prove the case in which $L$ is a convex-cocompact infinite-ended free group. A theorem of Koberda gives an abundance of suitable subgroups $L \leq \operatorname{MCG}(S)$ to which Theorem A.19 can be applied Kob12. Koberda shows that such subgroups are generic: if $n>1$ and $g_{1}, \ldots, g_{n}$ are infinite order elements of MCG $(S)$ that do not share a common power, at least one of which is a pseudo-Anosov, then $\left\langle g_{1}^{N}, \ldots g_{n}^{N}\right\rangle$ is an infinite-ended, irreducible subgroup of $\operatorname{MCG}(S)$ that is of type $F_{3}$ for $N$ sufficiently large.

However, there are many situations in which Question 1.1 fails to be true. In addition to Example 5.11 described above, we recall that Burger and Mozes constructed simple groups that are quasi-isometric to $F_{2} \times F_{2}$ [BM97. Theorem 1.4 demonstrates that a weakening of the notion of normal subgroup is frequently a quasi-isometry invariant. We now describe this property. 
Given an ambient group $G$, two subgroups $H$ and $K$ are said to be commensurable if the intersection $H \cap K$ has finite index in both $H$ and $K$. We define $\operatorname{Comm}_{G}(H)$ to be the subgroup of all $g \in G$ such that $H$ and $g H g^{-1}$ are commensurable. A subgroup $H \leq G$ is said to be almost normal if $G=\operatorname{Comm}_{G}(H)$, i.e. $H$ and $g H g^{-1}$ are commensurable for all $g \in G$. We denote this by $H \& G$. Almost normal subgroups have been studied extensively and are also known as inert subgroups, near normal subgroups and commensurated subgroups, e.g. [Bel93, Kro06, SW13].

Given a finitely generated group $G$ and a subgroup $H \backsim G$, the set $G / H$ of left $H$-cosets carries a proper metric, unique up to quasi-isometry, such that the natural left $G$ action is isometric. We refer to Section 3 for a definition of this metric. This space $G / H$ is called the quotient space. Indeed, in the case where $H$ is normal, the quotient space is quasi-isometric to the quotient group equipped with the word metric.

The map $p: G \rightarrow G / H$ taking $g$ to the left coset $g H$ is a coarse bundle in the sense of Why10. Showing that quasi-isometries preserve this coarse bundle structure is a key step in several celebrated quasi-isometric rigidity results, e.g. [FM99, [FM00], Why01, FM02a, [MSW03 and [EFW12]. This property is also called horizontal-respecting or height-respecting in the literature.

We consider the following variant of Question 1.1.

Question 1.2. Let $G$ be a finitely generated group containing an infinite almost normal subgroup $H$. If $G^{\prime}$ is a finitely generated group quasi-isometric to $G$, does $G^{\prime}$ also contain an almost normal subgroup $H^{\prime}$ a $G^{\prime}$ such that $H$ is quasi-isometric to $H^{\prime}$ and $G / H$ is quasi-isometric to $G^{\prime} / H^{\prime}$ ?

The Burger-Mozes groups, which were counterexamples to Question 1.1, are not counterexamples to Question 1.2, Indeed, the groups considered in [BM97] are of the form $\Gamma=F *_{H} K$, where $H, F$ and $K$ are finitely generated free groups such that $H$ has finite index in both $F$ and $K$. It is easy to see that $H$ is almost normal in $\Gamma$. The following proposition, which can be deduced from Theorem B.5, says that this is true, up to finite index, for all groups quasi-isometric to a product of non-abelian free groups.

Proposition 1.3. Suppose $G$ is a finitely generated group quasi-isometric to $F_{2} \times F_{2}$. Then $G$ contains a finite index subgroup $G^{\prime}$ and a finitely generated non-abelian free subgroup $H$ such that $H \backsim G^{\prime}$ and the quotient space $G^{\prime} / H$ is quasi-isometric to $F_{2}$.

This motivates the study of almost normal subgroups and their quotient spaces as a means to understanding the coarse geometry of finitely generated groups.

To show quasi-isometric rigidity of almost normal subgroups, we apply the coarse topological techniques developed in Mar18. To do this, we need to assume that the almost normal subgroup is a coarse Poincaré duality group. These were defined in KK05 and used extensively in the work of MosherSageev-Whyte MSW03, MSW11 and Papasoglu Pap07. We do not give a definition here, but note that the archetypal example of a coarse Poincaré duality group of dimension $n$, or coarse $P D_{n}$ group, is the fundamental group of a closed aspherical $n$-manifold. In particular, they include finitely 
generated free abelian groups and more generally, virtually polycyclic groups. The class of coarse Poincaré duality groups is closed under quasi-isometries.

We demonstrate that under suitable hypotheses, if a group contains an almost normal coarse $P D_{n}$ subgroup, then any group quasi-isometric to it also contains such a subgroup, and the associated coarse bundle structure is preserved by quasi-isometries.

Theorem 1.4. Fix $n \geq 1$. Let $G$ be a group of type $F_{n+1}$ and $H \backsim G$ be a coarse $P D_{n}$ subgroup with $e(G / H) \geq 3$. Suppose $G^{\prime}$ is a finitely generated group quasi-isometric to $G$. Then $G^{\prime}$ contains an almost normal coarse $P D_{n}$ subgroup $H^{\prime}$ such that $H$ is quasi-isometric to $H^{\prime}$ and $G / H$ is quasiisometric to $G^{\prime} / H^{\prime}$.

Theorem 1.4 is the main result of this article. We hope that this theorem and the methods used to prove it will have many more applications in quasi-isometric rigidity and classification results. Theorem 1.4 will be deduced from Theorem 4.1, a more technical and quantitative statement that is needed to prove the preceding applications. Theorem 1.4 and many of its applications actually hold under the weaker assumption that $G$ is only of type $F P_{n+1}$, a homological analogue of type $F_{n+1}$.

Recall that a subgroup $H \leq G$ is said to be characteristic if it is preserved by every automorphism of $G$. Analogously, we say that a subgroup $H \leq G$ of a finitely generated group is coarsely characteristic if it is coarsely preserved by every quasi-isometry of $G$, i.e. for every quasi-isometry $f: G \rightarrow G$, $H$ and $f(H)$ are at finite Hausdorff distance. Every coarsely characteristic subgroup is easily seen to be almost normal, see Proposition 3.12, A key step in our proof of Theorem 1.4 is a partial converse:

Theorem 1.5 (c.f. Lemma4.8). If $G$ is a group of type $F_{n+1}$ and $H$ a $G$ is a coarse $P D_{n}$ subgroup with $e(G / H) \geq 3$, then $H$ is coarsely characteristic.

The $e(G / H) \geq 3$ hypothesis in Theorem 1.4 is needed to apply the coarse topological techniques of [Mar18] and deduce that $H$ is coarsely characteristic. Indeed, both $\mathbb{Z}^{2}$ and $\mathbb{Z}^{3}$ have an infinite cyclic normal subgroup that is not coarsely characteristic. Similarly, if $M$ is a fibred hyperbolic 3-manifold, then the normal subgroup $\pi_{1}(S) \triangleleft \pi_{1}(M)$ is not coarsely characteristic.

Theorem 1.4 generalises [MSW03, Theorem 2], which proves the special case where the quotient space $G / H$ is quasi-isometric to a finite valence tree with infinitely many ends. To see this, we explain how to reformulate the hypotheses of Theorem 1.4 in terms of graphs of groups.

We first make the following observation: if a finitely generated group $G$ contains a normal subgroup $H \triangleleft G$ such that the quotient $K=G / H$ has more than one end, then $G$ splits as an amalgamated free product or HNN extension over a finite extension of $H$. This can be seen by applying Stallings' theorem to the quotient. If $K$ is assumed to be finitely presented, then by applying Dun85] to $K$, we see that $G$ splits as a finite graph of groups whose edge groups are finite extensions of $H$ and no vertex group splits over a finite extension of $H$. A similar statement holds in the situation when $H$ is only assumed to be almost normal:

Theorem 3.24. If $G$ is finitely presented and $H \backsim G$ is finitely generated, then $G$ is the fundamental group of a finite graph of groups such that: 
(1) every edge group is commensurable to $H$;

(2) every vertex group is finitely generated and doesn't split over a subgroup commensurable to $H$.

This can be deduced by applying Dunwoody accessibility to the quotient space $G / H$ [Dun85. Theorem 3.24 demonstrates a form of accessibility over a family of subgroups that are not necessarily small in the sense of [BF91]. Similar applications of Dunwoody's accessibility theorem to nonproper actions appear in the setting of totally disconnected locally compact groups [KM08. We can use Theorem 3.24 to reformulate Theorem 1.4.

Corollary 4.10. Let $G$ be a group of type $F_{n+1}$. Suppose that $G$ is the fundamental group of a graph of groups $\mathcal{G}$ with the following properties:

(1) the associated Bass-Serre tree has at least three ends;

(2) all conjugates of all edge groups are coarse $P D_{n}$ groups and are commensurable to one another;

(3) each vertex group is finitely generated and doesn't split over a subgroup commensurable to one of its incident edge groups.

If $G^{\prime}$ is a finitely generated group quasi-isometric to $G$, it is also the fundamental group of a graph of groups satisfying (11)-(3).

We can also prove a partial quasi-isometric classification theorem. If $\mathcal{G}$ is a graph of groups satisfying (11)-(3) as above, a vertex group is said to be essential if it is not commensurable to an incident edge group. If a vertex group $G_{v}$ is essential, any incident edge group $G_{e}$ is almost normal in $G_{v}$ with $e\left(G_{v} / G_{e}\right) \geq 1$. A quasi-isometry $f: G_{v} \rightarrow G_{v^{\prime}}^{\prime}$ is fibre-preserving if whenever $G_{e}$ and $G_{e^{\prime}}^{\prime}$ are edge groups incident to $G_{v}$ and $G_{v^{\prime}}^{\prime}, f$ sends left cosets of $G_{e}$ to within uniform finite Hausdorff distance of left cosets of $G_{e^{\prime}}^{\prime}$. In particular, if $f$ is fibre-preserving it induces a quasi-isometry $G_{v} / G_{e} \rightarrow G_{v^{\prime}}^{\prime} / G_{e^{\prime}}^{\prime}$ between quotient spaces.

Theorem 4.11. Let $G$ and $G^{\prime}$ be groups of type $F_{n+1}$ that are fundamental groups of finite graphs of groups $\mathcal{G}$ and $\mathcal{G}^{\prime}$ satisfying conditions (11)-(3) of Corollary 4.10. Suppose that $G$ and $G^{\prime}$ are quasi-isometric. Then for each essential vertex group $G_{v}$ of $\mathcal{G}$, there is an essential vertex group $G_{v^{\prime}}^{\prime}$ of $\mathcal{G}^{\prime}$ and a fibre-preserving quasi-isometry $G_{v} \rightarrow G_{v^{\prime}}^{\prime}$. Conversely, for each essential vertex $G_{v^{\prime}}^{\prime}$ of $\mathcal{G}^{\prime}$ there exists an essential vertex group $G_{v}$ of $\mathcal{G}$ and a fibre-preserving quasi-isometry $G_{v} \rightarrow G_{v^{\prime}}^{\prime}$.

A complete quasi-isometric classification for such groups is likely to be very difficult and is beyond the scope of this article. However, Farb-Mosher and Whyte have obtained quasi-isometric classification results in special cases [FM98, FM00, FM02a, Why01, Why10.

A solvable Baumslag-Solitar group is a group of the form $B S(1, n)=$ $\left\langle a, t \mid t a t^{-1}=a^{n}\right\rangle$ for some $n>1$. An important application of Theorem 1.4 is quasi-isometric rigidity for solvable Baumslag-Solitar groups, originally proven by Farb and Mosher [FM99].

Theorem 1.6 ([FM99]). A finitely generated group quasi-isometric to a solvable Baumslag-Solitar group has a finite index subgroup isomorphic to a solvable Baumslag-Solitar group. 
Proof. Using Corollary 4.10 and Theorem 4.11 we deduce that as $B S(1, n)$ is the fundamental group of a graph of two-ended groups, so is $G$. Moreover, $B S(1, n)$ has exponential growth and is solvable. Thus $G$ has exponential growth and is amenable. In particular, $G$ is not virtually $\mathbb{Z}^{2}$ and cannot contain a non-abelian free group. Thus $G$ is an ascending HNN extension of a 2-ended group, so contains a finite index solvable Baumslag-Solitar subgroup.

We claim no originality for the preceding proof, which is well-known to experts and can be easily be deduced from either MSW03, Pap05 or Pap07 without the use of Corollary 4.10. However, it is included to illustrate the power of the coarse topological techniques in this paper.

An important object in geometric group theory is the quasi-isometry group of a space, i.e. the group of all quasi-isometries modulo an appropriate equivalence relation. We can use the results of this paper to deduce the following:

Corollary 1.7. Let $G$ and $H$ be as in Theorem 1.4. Then there is a homomorphism $\mathrm{QI}(G) \rightarrow \mathrm{QI}(G / H)$.

This sort of observation is the starting point in the calculation of quasiisometry groups of solvable Baumslag-Solitar groups and surface-by-free groups in [FM99] and [FM02a].

We give an outline of the paper. Section 2 consists of background and preliminaries. In Section 3 we introduce the notion of coarse bundles and investigate their coarse geometric properties. In Section 4 we prove Theorems 1.4 and 4.11. Section 5 introduces the fibre distortion function, a quasi-isometry invariant that is used to prove Theorems 5.10 and 5.16. In Appendix $\mathrm{A}$ we give an account of Mosher's work on "fibre-respecting quasiisometries" of surface group extensions [Mos03a]. We then combine Mosher's results with the main result of this article to deduce Theorem A.19.

Our results build on work of Vavrichek, who uses Pap05 to prove part of Theorem 1.4 in the case where $H$ is two-ended. Many of the ideas in this article are inspired by work of Whyte [Why01, Why10, particularly in Section 5 .

The author would like to thank Panos Papasoglu, Michah Sageev and Ian Leary for helpful conversations.

\section{Preliminaries}

Much of this material is discussed in greater detail in [Mar18].

Coarse geometry. Let $(X, d)$ be a metric space. For $\emptyset \neq A \subseteq X$ and $x \in X$, we set $d(x, A):=\inf _{a \in A} d(x, a)$. We define $N_{r}(A):=\{x \in X \mid$ $d(x, A) \leq r\}$. If $A=\{a\}$, we also denote $N_{r}(A)$ by $N_{r}(a)$. We say that $X$ has bounded geometry if there is a function $M: \mathbb{R}_{\geq 0} \rightarrow \mathbb{R}_{\geq 0}$ such that for all $r \in \mathbb{R}_{\geq 0}$ and $x \in X,\left|N_{r}(x)\right| \leq M(r)$. The Hausdorff distance between two subsets $A, B \subseteq X$ is defined to be

$$
d_{\text {Haus }}(A, B):=\inf \left\{r \in \mathbb{R} \mid A \subseteq N_{r}(B) \text { and } B \subseteq N_{r}(A)\right\} .
$$


In general, this infimum is not achieved. However, the infimum is achieved when $X$ is a finitely generated group equipped with the word metric, since then $d(X \times X) \subseteq \mathbb{N}$ is discrete.

Recall that a function $\phi: \mathbb{R}_{\geq 0} \rightarrow \mathbb{R}_{\geq 0}$ is proper if the inverse images of compact sets are compact.

Definition 2.1. Let $\left(X, d_{X}\right)$ and $\left(Y, d_{Y}\right)$ be metric spaces and let $\eta, \phi$ : $\mathbb{R}_{\geq 0} \rightarrow \mathbb{R}_{\geq 0}$ be proper non-decreasing functions. A function $f: X \rightarrow Y$ is a $(\eta, \phi)$-coarse embedding if for all $x, x^{\prime} \in X$,

$$
\eta\left(d_{X}\left(x, x^{\prime}\right)\right) \leq d_{Y}\left(f(x), f\left(x^{\prime}\right)\right) \leq \phi\left(d_{X}\left(x, x^{\prime}\right)\right) .
$$

We say that $f$ is a coarse embedding if it is an $(\eta, \phi)$-coarse embedding for some $\eta$ and $\phi$. We say that $\eta$ and $\phi$ are the distortion functions of $f$. A coarse equivalence is a coarse embedding $f: X \rightarrow Y$ such that $N_{A}(f(X))=Y$ for some $A \geq 0$.

Remark 2.2. For each proper non-decreasing function $\phi: \mathbb{R}_{\geq 0} \rightarrow \mathbb{R}_{>0}$, we define another proper non-decreasing function $\widetilde{\phi}: \mathbb{R}_{\geq 0} \rightarrow \mathbb{R}_{\geq 0}$ by $\widetilde{\phi}(R):=$ $\sup \left(\phi^{-1}([0, R])\right)$. This can be thought of as a sort of inverse to $\phi$ in the sense that if $\phi(S) \leq R$, then $S \leq \widetilde{\phi}(R)$, and if $R<\phi(S)$, then $\widetilde{\phi}(R) \leq S$.

We are particularly interested in the following subclass of coarse embeddings:

Definition 2.3. Let $\left(X, d_{X}\right)$ and $\left(Y, d_{Y}\right)$ be metric spaces and let $K \geq 1$ and $A \geq 0$. A function $f: X \rightarrow Y$ is a $(K, A)$-quasi-isometry if the following hold:

(1) for all $x, x^{\prime} \in X, \frac{1}{K} d_{X}\left(x, x^{\prime}\right)-A \leq d_{Y}\left(f(x), f\left(x^{\prime}\right)\right) \leq K d_{X}\left(x, x^{\prime}\right)+A$;

(2) for all $y \in Y$, there exists an $x \in X$ with $d_{Y}(f(x), y) \leq A$.

We say that $f$ is a quasi-isometry if there exist $K \geq 1$ and $A \geq 0$ such that $f$ is a $(K, A)$-quasi-isometry.

We say that $f, g: X \rightarrow Y$ are $A$-close if $\sup _{x \in X} d(f(x), g(x)) \leq A$, and we say that $f$ and $g$ are close if they are $A$-close for some $A<\infty$. A coarse inverse to $f: X \rightarrow Y$ is a function $\bar{f}: Y \rightarrow X$ such that $f \circ \bar{f}$ and $\bar{f} \circ f$ are close to $\operatorname{id}_{Y}$ and $\operatorname{id}_{X}$. Every quasi-isometry has a coarse inverse. Being close defines an equivalence relation on the set of all quasi-isometries from $X$ to $X$, and we let $[f]$ denote the equivalence class containing $f$. We can thus define a group $\mathrm{QI}(X):=\{[f] \mid f: X \rightarrow X$ is a quasi-isometry $\}$ with group operation $[f] \cdot[g]=[f \circ g]$.

A key idea in geometric group theory is that a finitely generated group can be equipped with the word metric with respect to a finite generating set. It is an easy exercise to see that if we equip a group with two different word metrics with respect to two finite generating sets, these metrics are quasi-isometric. This motivates the study of groups up to quasi-isometry. Throughout this article, whenever a finitely generated group is considered as a metric space, it will always be equipped with the word metric with respect to some finite generating set unless explicitly stated.

The following lemma motivates our interest in more general coarse embeddings whose distortion functions are not necessarily affine. 
Lemma 2.4 (e.g. see Roe03, Remark 1.20]). Let $G$ be a finitely generated group containing a finitely generated subgroup $H \leq G$. Let $d_{H}$ and $d_{G}$ be word metrics of $H$ and $G$ with respect to finite generating sets. Then the inclusion $\left(H, d_{H}\right) \rightarrow\left(G, d_{G}\right)$ is a coarse embedding.

A space is said to be quasi-geodesic if it is quasi-isometric to a geodesic metric space. For example, a finitely generated group $G$ equipped with the word metric is quasi-geodesic but not geodesic (unless $G$ is the trivial group). If a metric space is quasi-geodesic, it can be approximated by a simplicial complex known as the Rips complex.

Definition 2.5. Given a metric space $(X, d)$ and a parameter $r \geq 0$, the Rips complex $P_{r}(X)$ is a simplicial complex with vertex set $X$ such that $\left\{x_{0}, \ldots, x_{n}\right\}$ spans a simplex if $d\left(x_{i}, x_{j}\right) \leq r$ for all $1 \leq i, j \leq n$. The Rips graph $P_{r}^{1}(X)$ is the 1-skeleton of $P_{r}(X)$.

If $P_{r}^{1}(X)$ is connected, it can be equipped with the induced path metric in which all edges have length 1 . The following proposition relates this metric to the original metric on $X$. A $t$-chain of length $n$ from $x$ to $y$ is a sequence of points $x=x_{0}, x_{1}, \ldots, x_{n}=y$ such that $d\left(x_{i-1}, x_{i}\right) \leq t$ for $1 \leq i \leq n$.

Proposition 2.6 (special case of Proposition 2.5 of [Mar18]). Let $(X, d)$ be a metric space. The following are equivalent:

(1) $X$ is a quasi-geodesic metric space;

(2) there exist constants $K \geq 1$ and $A, t \geq 0$ such that any $x, y \in X$ can be joined by a $t$-chain of length at most $K d(x, y)+A$;

(3) there exists a $t \geq 0$ such that for all $r \geq t$, the Rips graph $P_{r}^{1}(X)$ is connected and the inclusion $X \rightarrow P_{r}^{1}(X)$ is a quasi-isometry.

The following lemma gives an intrinsic characterisation of the image of a coarsely embedded quasi-geodesic metric space.

Lemma 2.7. Let $Y$ be a metric space and $W \subseteq Y$. The following are equivalent:

(1) There is a quasi-geodesic metric space $X$, unique up to quasi-isometry, and a coarse embedding $f: X \rightarrow Y$ such that $d_{\text {Haus }}(f(X), W)<\infty$.

(2) There exists a $t \geq 0$ and a proper non-decreasing function $\eta: \mathbb{R}_{>0} \rightarrow$ $\mathbb{R}_{>0}$ such that every $x, y \in W$ can be joined by a $t$-chain in $W$ of length at most $\eta(d(x, y))$.

Moreover, $t$ and $\eta$ in (2) depend only the distortion functions of $f: X \rightarrow Y$ and vice versa.

Proof. We equip $W \subseteq Y$ with the subspace metric.

(11) $\Longrightarrow$ (2): Since $d_{\text {Haus }}(f(X), W)<\infty$, we can modify $f$ by a bounded amount so that $f(X) \subseteq W$. Thus $f$ can be thought of as a coarse equivalence $f: X \rightarrow W$. Since $W$ is coarsely equivalent to a geodesic metric space, Proposition 2.5 of Mar18 tells us that $W$ satisfies (2).

(2) $\Longrightarrow$ (11): It follows from Proposition 2.5 of Mar18 and (2) that $W$ is coarsely equivalent to a (quasi-)geodesic metric space $X$, and so there is a coarse embedding $f: X \rightarrow Y$ with image $W$. If $W$ is also coarsely equivalent to a quasi-geodesic metric space $X^{\prime}$, then $X$ and $X^{\prime}$ are quasi-geodesic metric spaces that are coarsely equivalent. Since every coarse equivalence between 
quasi-geodesic metric spaces is a quasi-isometry, we conclude that $X$ and $X^{\prime}$ are quasi-isometric.

By a slight abuse of notation, we say that $W \subseteq Y$ is coarsely embedded in $Y$ if it satisfies one of the two equivalent conditions in Lemma 2.7.

Most of the spaces that we work with are discrete geodesic metric spaces in the following sense:

Definition 2.8. Let $(X, d)$ be a metric space. A discrete geodesic between $x, y \in X$ is a sequence of points $x=x_{0}, x_{1}, \ldots, x_{n}=y$ such that $d\left(x_{i}, x_{j}\right)=$ $|i-j|$. We say that $X$ is a discrete geodesic metric space if every pair of points can be joined by a discrete geodesic.

Remark 2.9. If $X$ is quasi-geodesic, it follows from Proposition 2.6 that $X$ can be remetrised by identifying it with the vertex set of the Rips graph $P_{r}^{1}(X)$ for large enough $r$. This new metric is quasi-isometric to the original metric and is a discrete geodesic metric space. Thus every quasi-geodesic metric space can be remetrised to be a discrete geodesic metric space. Moreover, any group action by isometries on $X$ induces a group action by isometries on the remetrised discrete geodesic space.

We will require the following technical result:

Proposition 2.10 (cf. FM00, Lemma 2.1]). Let $X, Y, Z$ and $W$ be discrete geodesic metric spaces and let $s: Z \rightarrow G$ and $s^{\prime}: W \rightarrow Y$ be coarse embeddings. Suppose that $f: X \rightarrow Y$ is a quasi-isometry and there is a constant $A$ such that $d_{\text {Haus }}\left(f(\operatorname{im}(s)), \operatorname{im}\left(s^{\prime}\right)\right) \leq A$. Then there is a quasi-isometry $g: Z \rightarrow W$ with $\sup _{z \in Z} d_{Y}\left(s^{\prime} \circ g(z), f \circ s(z)\right) \leq A$, whose quasi-isometry constants depend only on $A$ and the distortion functions associated to $f, s$ and $s^{\prime}$.

Coarse separation and ends. In what follows, we assume that $(X, d)$ is a discrete geodesic bounded geometry metric space. Given a subset $C \subseteq X$, we define the coarse boundary $\partial C$ to be $\{x \in X \mid d(x, C)=1\}$. We say that $C \subseteq X$ is a coarse complementary component of $W \subseteq X$ if there exists an $A \geq 0$ such that $\partial C \subseteq N_{A}(W)$. The intersection, complement, union and symmetric difference of two coarse complementary components of $W$ is again a coarse complementary component of $W$, as shown in Mar18.

A coarse complementary component is said to be deep if it is not contained in $N_{A}(W)$ for any $A \geq 0$. Otherwise it is said to be shallow. A collection of coarse complementary components $\left\{C_{i}\right\}_{i \in I}$ is said to be coarse disjoint if $C_{i} \cap C_{j}$ is shallow for every distinct $i, j \in I$. We say that $W$ coarsely $n$-separates $G$ if there exist $n$ deep, coarse disjoint, coarse complementary components of $W$ in $G$. Coarse complementary components and coarse separation are quasi-isometry invariants. We caution the reader that coarse complementary components are not necessarily coarsely connected.

The following definition is slightly non-standard, but agrees with other definitions of ends.

Definition 2.11. Let $X$ be a discrete geodesic, bounded geometry metric space. We define the number of ends of $X$ to be

$$
e(X):=\sup \{n \in \mathbb{N} \mid X \text { is } n \text {-separated by a point }\} \text {. }
$$


In the case where $W$ is a subgroup of $G$, coarse complementary components and coarse $n$-separation can be characterised in terms of more classical notions of almost invariant subsets and Kropholler-Roller number of relative ends, denoted $\tilde{e}(-,-)$ KR89]. Although we do not define these notions here, the following proposition can be thought of as a definition for the purposes of this article.

Proposition 2.12 ([Mar18, Propositions 5.14 and 5.15]). Let $G$ be a finitely generated group and $H \leq G$ be a subgroup.

(1) $C \subseteq G$ is a coarse complementary component of $H$ if and only if it is an $H$-almost invariant subset;

(2) $H$ coarsely $n$-separates $G$ if and only if $\tilde{e}(G, H) \geq n$.

Bowditch's coends and Geoghegan's filtered ends are alternative interpretations of relative ends of groups [Bow02, Geo08].

Finiteness properties and coarse topology. We define finiteness properties of groups, which generalise the notion of being finitely generated and presented. All homology and cohomology will be taken with coefficients over $\mathbb{Z}_{2}$.

Definition 2.13. We say that a group $G$ has type $F_{n}$ if there exists a $K(G, 1)$ with finite $n$-skeleton. We say that a group $G$ has type $F P_{n}$ over a ring $R$ if the trivial $R G$ module $R$ has a projective resolution $P_{\bullet} \rightarrow R$ with $P_{i}$ finitely generated for $i \leq n$.

Throughout this article, it will be assumed that type $F P_{n}$ means type $F P_{n}$ over $\mathbb{Z}_{2}$. Finiteness properties can be characterised geometrically. To see this, we need the following definitions:

Definition 2.14. Let $X$ be a metric space and $n \geq 0$.

(1) We say that $X$ is coarsely uniformly $n$-connected if for all $i, r$, there exists a $j=j(i) \geq i$ and $s=s(i, r) \geq r$ such that for all $k \leq n$ and $x \in X$, the map

$$
\pi_{k}\left(P_{i}\left(N_{r}(x)\right)\right) \rightarrow \pi_{k}\left(P_{j}\left(N_{s}(x)\right)\right),
$$

induced by inclusion, is zero.

(2) We say that $X$ is coarsely uniformly $n$-acyclic if for all $i, r$, there exists a $j=j(i) \geq i$ and $s=s(i, r) \geq r$ such that for all $k \leq n$ and $x \in X$, the map

$$
\tilde{H}_{k}\left(P_{i}\left(N_{r}(x)\right)\right) \rightarrow \tilde{H}_{k}\left(P_{j}\left(N_{s}(x)\right)\right),
$$

induced by inclusion, is zero.

As these properties are quasi-isometry invariants, the following theorem then demonstrates that finiteness properties are quasi-isometry invariants.

Theorem 2.15 ([KK05, [DK18]). Let $G$ be a finitely generated group.

(1) $G$ is of type $F_{n}$ if and only if it is coarsely uniformly $(n-1)$ connected.

(2) $G$ is of type $F P_{n}$ if and only if it is coarsely uniformly $(n-1)$-acyclic. 
The definition of coarse connectedness and acyclicity is somewhat difficult to work with as the property cannot be phrased in terms of a single Rips complex. However, in the case $n=1$, things are simpler:

Lemma 2.16. Suppose $X$ is a coarsely 1-acyclic, discrete geodesic metric space. Then there exists an $r$ such that $P_{r}(X)$ is 1-acyclic.

Proof. As $X$ is coarsely 1-acyclic, there is an $r \geq 1$ such that for $k=$ 0,1 , the map $\tilde{H}_{k}\left(P_{1}(X)\right) \rightarrow \tilde{H}_{k}\left(P_{r}(X)\right)$, induced by inclusion, is zero. Let $\sigma=[x, y]$ be a 1 -simplex in $P_{r}(X)$. There exists a discrete geodesic $x=$ $x_{0}, x_{1}, \ldots, x_{n}=y$ from $x$ to $y$ in $X$, where $n=d(x, y) \leq r$. Thus $d\left(x_{i}, x_{j}\right)=$ $|i-j| \leq r$ for all $1 \leq i, j \leq n$. It follows that for each $i, \omega_{i}:=\left[x_{0}, x_{i-1}, x_{i}\right]$ is a 2-simplex in $P_{r}(X)$, and so $\partial\left(\sum_{i=1}^{n} \omega_{i}\right)=\sum_{i=1}^{n}\left[x_{i-1}, x_{i}\right]-\sigma$. It follows that every reduced 1-cycle in $P_{r}(X)$ is homologous to one in $P_{1}(X)$, thus $P_{r}(X)$ is 1-acyclic.

We make use of the following class of spaces, defined in [MSW03, that allow us to apply both topological and metric arguments.

Definition 2.17. A metric cell complex consists of the pair $(X, \mathbf{X})$, where $\mathbf{X}$ is a connected cell complex, $X$ is a bounded geometry metric space called the control space, and there is a function $p$ from the set of cells of $\mathbf{X}$ to $X$ such that:

(1) for each $d \in \mathbb{N}$, there is a number $N_{d}$ such that for every $x \in X$, $p^{-1}(x)$ contains at most $N_{d} d$-cells;

(2) for each $d \in \mathbb{N}$, there is a number $M_{d}$ such that for every $d$-cell $e$ with attaching map $\phi_{e},\{p(e)\} \cup\left\{p(f) \mid f \cap \operatorname{im}\left(\phi_{e}\right) \neq \emptyset\right\}$ has diameter at most $M_{d}$

(3) $p\left(\mathbf{X}^{(0)}\right)=X$.

We say that a group $G$ acts on $(X, \mathbf{X})$ if $G$ acts on both $X$ and $\mathbf{X}$ and the map $p$ is $G$-equivariant. Such an action is free if the corresponding action of $G$ is free on $X$.

Almost all examples of metric cell complexes arising in this paper come from the following construction:

Example 2.18. Suppose a finitely generated group $G$, equipped with the word metric, acts freely and cocompactly on a cell complex $\mathbf{X}$. We define $p$ arbitrarily on a transversal for the set of $G$-orbits of cells and extend $p$ equivariantly. Thus $(G, \mathbf{X})$ is a metric cell complex admitting a free $G$ action.

Remark 2.19. In fact, there is a much more general class of objects called metric complexes that were defined in [KK05] and used extensively in Mar18]. For readability and conciseness, we will not define these objects here. However, it will be clear to those familiar with the theory of metric complexes that all arguments in this article extend to the setting of metric complexes.

Definition 2.20. Let $(X, \mathbf{X})$ be a metric cell complex. Given a subset $A \subseteq Y$, we define $\mathbf{X}[A]$ to be the largest subcomplex of $\mathbf{X}$ such that $p(e) \in A$ for every cell $e$ in $\mathbf{X}[A]$. We say that $(X, \mathbf{X})$ is uniformly $n$-connected if for all $r$, there exists an $s \geq r$ such that for all $x \in X$ and $k \geq n$, the map

$$
\pi_{k}\left(\mathbf{X}\left[N_{r}(x)\right]\right) \rightarrow \pi_{k}\left(\mathbf{X}\left[N_{s}(x)\right]\right),
$$


induced by inclusion, is zero.

There is a corresponding notion of uniform $n$-acyclicity for metric cell complexes and more generally, metric complexes.

Proposition 2.21 ([Mar18, Proposition 3.20]). Let $(X, d)$ be a bounded geometry metric space. Then:

(1) $X$ is coarsely uniformly $n$-connected if and only if it is the control space of a uniformly $n$-connected metric cell complex.

(2) $X$ is coarsely uniformly $n$-acyclic if and only if it is the control space of a uniformly n-acyclic metric complex.

Moreover, if $X=G$ is a finitely generated group equipped with the word metric, we can assume that the resulting metric cell complex in (1) and (2) admits a free $G$-action.

Given a metric complex $(X, \mathbf{X})$, we define the support of (cellular) chains and cochains as follows. If $\left\{\sigma_{i}\right\}_{i \in I}$ is the set of $k$-cells of $\mathbf{X}$, then the support of a chain $\sum_{i \in I} n_{i} \sigma_{i} \in C_{k}(\mathbf{X})$ is defined to be $\left\{p\left(\sigma_{i}\right) \mid n_{i} \neq 0\right\}$. Similarly, the support of a cochain $\alpha \in C^{k}(\mathbf{X})$ is defined to be $\left\{p\left(\sigma_{i}\right) \mid \alpha\left(\sigma_{i}\right) \neq 0\right\}$. We define compactly supported cochains as cochains $\alpha$ with $\operatorname{supp}(\alpha)$ finite. Compactly supported cochains form a subcochain complex $C_{c}^{\bullet}(\mathbf{X})$ and we thus define the compactly supported cohomology $H_{c}^{*}(\mathbf{X})$ as the cohomology of this cochain complex.

In Mar18, the author defines a coarse version of cohomology; see also [Roe93] and KK05. The definition can be extended to coarsely uniformly $(n-1)$-acyclic metric spaces using the theory of metric complexes.

Definition 2.22. Let $X$ be a bounded geometry, coarsely uniformly $(n-1)$ connected metric space. Then for $k \leq n, H_{\text {coarse }}^{k}(X):=\operatorname{ker}\left(H_{c}^{k}(\mathbf{X}) \rightarrow\right.$ $\left.H^{k}(\mathbf{X})\right)$, where $\mathbf{X}$ is a uniformly $(n-1)$-connected metric complex with control space $X$.

This is invariant under quasi-isometries. Moreover, a coarse embedding $A \rightarrow X$ induces a map $H_{\text {coarse }}^{n}(X) \rightarrow H_{\text {coarse }}^{n}(A)$. When $G$ is a group of type $F_{n}, H_{\text {coarse }}^{k}(G)=H^{k}\left(G, \mathbb{Z}_{2} G\right)$ for $k \leq n$ (recall all cohomology is taken with coefficients in $\left.\mathbb{Z}_{2}\right)$.

We can now define coarse $P D_{n}$ spaces.

Definition 2.23. Let $(X, \mathbf{X})$ be a uniformly acyclic metric cell complex. We say that $\mathbf{X}$ is a coarse $P D_{n}$ complex if there exists a number $R$, chain maps

$$
C_{c}^{n-\bullet}(\mathbf{X}) \stackrel{P}{\rightarrow} C_{\bullet}(\mathbf{X}) \text { and } C_{\bullet}(\mathbf{X}) \stackrel{\bar{P}}{\rightarrow} C_{c}^{n-\bullet}(\mathbf{X}),
$$

and chain homotopies $\bar{P} \circ P \stackrel{\Phi}{\simeq} \operatorname{id}_{C_{c}^{n-\bullet}(\mathbf{X})}$ and $P \circ \bar{P} \stackrel{\bar{\Phi}}{\simeq} \operatorname{id}_{C \cdot(\mathbf{X})}$ such that:

(1) for every chain $\sigma, \operatorname{supp}(\bar{P}(\sigma))$ and $\operatorname{supp}(\bar{\Phi}(\sigma))$ are contained in $N_{R}(\operatorname{supp}(\sigma))$

(2) for every cochain $\alpha, \operatorname{supp}(P(\alpha))$ and $\operatorname{supp}(\Phi(\alpha))$ are contained in $N_{R}(\operatorname{supp}(\alpha))$.

A metric space is said to be coarse $P D_{n}$ if it is the control space of some coarse $P D_{n}$ complex. A coarse $P D_{n}$ group is a finitely generated group that is a coarse $P D_{n}$ space. 
If $X$ is a coarse $P D_{n}$ space, then $H_{\text {coarse }}^{n}(X) \cong \mathbb{Z}_{2}$ and $H_{\text {coarse }}^{k}(X)=0$ for $k \neq n$. We refer to [KK05, for a more thorough treatment of coarse $P D_{n}$ complexes and spaces. We recall the following useful fact about coarse $P D_{n}$ spaces, generalising an earlier result of Farb-Schwartz [FS96].

Theorem 2.24 (Packing Theorem, KK05, Part 4 of Theorem 7.7]). If $W$ and $W^{\prime}$ are coarse $P D_{n}$ subspaces of $X$ and $W \subseteq N_{R}\left(W^{\prime}\right)$ for some $R \geq 0$, then there exists an $R^{\prime} \geq 0$ such that $W^{\prime} \subseteq N_{R^{\prime}}(W)$.

We now define the technical hypothesis in the main theorem of [Mar18]:

Definition 2.25. Let $X$ be a bounded geometry, coarsely uniformly $n$ acyclic metric space containing a coarse $P D_{n}$ subspace $W \subseteq X$. A coarse complementary component $C$ of $W$ is said to be essential if the map $H_{\text {coarse }}^{n}(C \cup$ $W) \rightarrow H_{\text {coarse }}^{n}(W)$, induced by inclusion, is zero.

Every essential coarse complementary component is deep. Although the converse is not true, there are many instances in which deep and essential coarse complementary components are equivalent, e.g. Mar18, Proposition 1.2] and Proposition 4.3, We refer to [Mar18] for a thorough of discussion of essential components.

In [KK05], Kapovich and Kleiner prove a coarse version of Jordan separation: a coarse $P D_{n}$ space $W$ coarsely separates a coarse $P D_{n+1}$ space into precisely two deep coarse complementary components. We say that such a component is a coarse $P D_{n+1}$ half-space with boundary $W$. We will make use of the following criterion for showing coarse complementary components are essential.

Lemma 2.26 ([Mar18, Corollary 6.7]). Let $X$ be a bounded geometry, coarsely uniformly $n$-acyclic metric space containing a coarse $P D_{n}$ subspace $W \subseteq X$. If a coarse complementary component $C$ of $W$ contains a coarse $P D_{n+1}$ half-space with boundary $W$, then $C$ is essential.

We now state part of the main theorem of [Mar18]:

Theorem 2.27 ([Mar18]). Let $G$ be a group of type $F P_{n+1}$. If $W \subseteq G$ is a coarse $P D_{n}$ subspace that coarsely separates $G$ into three essential coarse complementary components, then there is a subgroup $H \leq G$ with $d_{\text {Haus }}(H, W)<\infty$.

To prove Theorem 1.4, we not only need Theorem 2.27, but some of the ingredients used to prove it. Specifically, we require the following lemma.

Lemma 2.28 ([Mar18, Proposition 5.19 and Lemma 6.13]). Let $X$ be a bounded geometry metric space and let $(X, \mathbf{X})$ be a uniformly $n$-connected metric cell complex. Suppose $W \subseteq X$ is a coarse $P D_{n}$ subspace and that $C_{1}$, $C_{2}$ and $C_{3}$ are coarse disjoint, essential, coarse complementary components of $W$. Then there exist constants $D, E \geq 0$ such that:

(1) there exist non-zero, distinct cohomology classes

$$
\left[\alpha_{1}\right],\left[\alpha_{2}\right] \in \operatorname{ker}\left(H_{c}^{n+1}(\mathbf{X}) \rightarrow H^{n+1}(\mathbf{X})\right)
$$

such that for every $x \in W,\left[\alpha_{i}\right]$ can be supported by a cocycle supported in $N_{D}(x)$ for $i=1,2$; 
(2) $\alpha_{i}$ can be represented by a cocycle supported in $C_{i} \backslash N_{E}(W)$ for $i=$ 1,2

(3) if $[\omega] \in \operatorname{ker}\left(H_{c}^{n+1}(\mathbf{X}) \rightarrow H^{n+1}(\mathbf{X})\right)$ is a cohomology class represented by cocycles supported in any two of $C_{1} \backslash N_{E}(W), C_{2} \backslash N_{E}(W)$ and $C_{3} \backslash N_{E}(W)$, then $[\omega]=0$.

\section{Coarse Bundles}

In this section we introduce coarse bundles, one of the key concepts of this article. Similar definitions appear in [FM00, KK05] and Why10.

Definition 3.1. Let $(X, d),\left(F, d_{F}\right)$ and $\left(B, d_{B}\right)$ be bounded geometry, quasigeodesic metric spaces. We say that $X$ is a coarse bundle over $B$ with fibre $F$ if there are constants $K \geq 1, A, E \geq 0$, proper non-decreasing functions $\eta, \phi: \mathbb{R}_{\geq 0} \rightarrow \mathbb{R}_{\geq 0}$ and a map $p: X \rightarrow B$ such that:

(1) $p$ is $(K, A)$-coarse Lipschitz, i.e. for all $x, x^{\prime} \in X$,

$$
d_{B}\left(p(x), p\left(x^{\prime}\right)\right) \leq K d\left(x, x^{\prime}\right)+A ;
$$

(2) for all $b \in B, D_{b}:=p^{-1}\left(N_{E}(b)\right)$ is known as a fibre of $X$ and there is an $(\eta, \phi)$-coarse embedding $s_{b}: F \rightarrow X$ with

$$
\operatorname{im}\left(s_{b}\right) \subseteq D_{b} \subseteq N_{A}\left(\operatorname{im}\left(s_{b}\right)\right) ;
$$

(3) for all $b, b^{\prime} \in B, d_{\text {Haus }}\left(D_{b}, D_{b^{\prime}}\right) \leq K d_{B}\left(b, b^{\prime}\right)+A$.

When the above holds, we say that $p: X \rightarrow B$ is a $(K, A, E)$-coarse bundle, or simply a coarse bundle. We call $X$ the total space, $B$ the base space and $F$ the fibre.

Remark 3.2. Given a coarse bundle $p: X \rightarrow B$ and a fibre $D_{b}$ as above, the space $F$ can be recovered from $D_{b}$ up to quasi-isometry using Lemma 2.7. Thus there is no ambiguity in referring to $D_{b}$ as a fibre, as it is just a distorted copy of $F$ sitting inside $X$.

Proposition 3.3. Suppose that $p: X \rightarrow B$ is a coarse bundle with fibre $F$ and that $f: Y \rightarrow X$ is a quasi-isometry. Then $p \circ f: Y \rightarrow B$ is also a coarse bundle with fibre $F$.

Proposition 3.3 can be deduced from Lemma 3.10. Most of the coarse bundles that we are interested in satisfy the following:

Definition 3.4. Let $p: X \rightarrow B$ be a coarse bundle. A quasi-isometry $f: X \rightarrow X$ is said to be $A$-fibre-preserving if for every $b \in B$, there exists a $b^{\prime} \in B$ such that $d_{\text {Haus }}\left(f\left(D_{b}\right), D_{b^{\prime}}\right) \leq A$. We say that $p: X \rightarrow B$ is quasihomogeneous if there exist constants $K \geq 1$ and $A \geq 0$ such that for all $x, y \in X$, there is an $A$-fibre-preserving $(K, A)$-quasi-isometry $f: X \rightarrow X$ such that $d(f(x), y) \leq A$.

Quasi-homogeneity of coarse bundles is easily seen to be a quasi-isometry invariant. The following lemma follows from the preceding definitions.

Lemma 3.5. Let $p: X \rightarrow B$ be a quasi-homogeneous coarse bundle and $x_{0} \in X$ be a basepoint. Then there exist constants $K \geq 1$ and $A \geq 0$ such that for every $x \in X$ : 
(1) There is an A-fibre-preserving $(K, A)$-quasi-isometry $f_{x}: X \rightarrow X$ such that $d\left(f_{x}(x), x_{0}\right) \leq A$;

(2) $f_{x}$ has a coarse inverse $\overline{f_{x}}: X \rightarrow X$ which is also an A-fibrepreserving $(K, A)$-quasi-isometry such that $\overline{f_{x}} \circ f_{x}$ and $f_{x} \circ \overline{f_{x}}$ are $A$-close to the identity.

Arbitrary quasi-isometries between coarse bundles don't preserve the coarse bundle structure. However, if two coarse bundles are quasi-isometric via a fibre-preserving quasi-isometry, then it follows from Proposition 2.10 that the fibres are quasi-isometric. Moreover, the following lemma demonstrates that the base spaces are quasi-isometric:

Lemma 3.6. Let $p: X \rightarrow B$ and $p^{\prime}: Y \rightarrow B^{\prime}$ be coarse bundles. Suppose $f: X \rightarrow Y$ is a $(K, A)$-quasi-isometry that is A-fibre-preserving. Then $f$ induces a $\left(K^{\prime}, A^{\prime}\right)$-quasi-isometry $\hat{f}: B \rightarrow B^{\prime}$ such that $d_{\text {Haus }}\left(D_{\hat{f}(b)}, f\left(D_{b}\right)\right) \leq$ $A$. The constants $K^{\prime}$ and $A^{\prime}$ depend only on $K$ and $A$.

Proof. By hypothesis, such a function $\hat{f}$ exists and is coarsely well-defined. We need only show that $\hat{f}$ is a quasi-isometry. By increasing $K$ and $A$ if necessary, we may assume that $p: X \rightarrow B$ and $p^{\prime}: X^{\prime} \rightarrow B^{\prime}$ are $(K, A, E)$ coarse bundles. We fix $b, b^{\prime} \in B$ and pick $x \in D_{b}$ and $x^{\prime} \in D_{b^{\prime}}$ such that $d\left(x, x^{\prime}\right) \leq d_{\text {Haus }}\left(D_{b}, D_{b^{\prime}}\right) \leq K d_{B}\left(b, b^{\prime}\right)+A$. We also observe that

$$
d_{B}\left(b, b^{\prime}\right) \leq d_{B}\left(p(x), p\left(x^{\prime}\right)\right)+2 E \leq K d\left(x, x^{\prime}\right)+A+2 E .
$$

It follows from the definition of $\hat{f}$ that there is a $y \in D_{\hat{f}(b)}$ with $d(y, f(x)) \leq$ $A$. Thus $d_{B^{\prime}}\left(\hat{f}(b), p^{\prime}(f(x))\right) \leq K A+A+E$. The same argument shows that $d_{B^{\prime}}\left(\hat{f}\left(b^{\prime}\right), p^{\prime}\left(f\left(x^{\prime}\right)\right)\right) \leq K A+A+E$, and so

$$
\begin{aligned}
d_{B^{\prime}}\left(\hat{f}(b), \hat{f}\left(b^{\prime}\right)\right) & \leq d\left(p^{\prime}(f(x)), p^{\prime}\left(f\left(x^{\prime}\right)\right)\right)+2(K A+A+E) \\
& \leq K d\left(f(x), f\left(x^{\prime}\right)\right)+2 K A+3 A+2 E \\
& \leq K^{2} d\left(x, x^{\prime}\right)+3 K A+3 A+2 E \\
& \leq K^{3} d_{B}\left(b, b^{\prime}\right)+K^{2} A+3 K A+3 A+2 E .
\end{aligned}
$$

We observe that $d_{\text {Haus }}\left(D_{b}, D_{b^{\prime}}\right) \leq K d_{\text {Haus }}\left(f\left(D_{b}\right), f\left(D_{b^{\prime}}\right)\right)+K A$ and so

$$
\begin{aligned}
d_{B}\left(b, b^{\prime}\right) & \leq K d_{X}\left(x, x^{\prime}\right)+A+2 E \\
& \leq K d_{\text {Haus }}\left(D_{b}, D_{b^{\prime}}\right)+A+2 E \\
& \leq K^{2} d_{\text {Haus }}\left(f\left(D_{b}\right), f\left(D_{b^{\prime}}\right)\right)+K^{2} A+A+2 E \\
& \leq K^{2} d_{\text {Haus }}\left(D_{\widehat{f}(b)}, D_{\widehat{f}\left(b^{\prime}\right)}\right)+3 K^{2} A+A+2 E \\
& \leq K^{3} d_{B^{\prime}}\left(\widehat{f}(b), \widehat{f}\left(b^{\prime}\right)\right)+4 K^{2} A+A+2 E .
\end{aligned}
$$

Finally, for each $b^{\prime} \in B^{\prime}$ there is some $z^{\prime} \in D_{b^{\prime}}$ and $z \in X$ with $d\left(f(z), z^{\prime}\right) \leq$ $A$. Let $b:=p(z)$. Since $z \in D_{b}$ and $d_{\text {Haus }}\left(D_{\hat{f}(b)}, f\left(D_{b}\right)\right) \leq A$, there is some $y \in D_{\hat{f}(b)}$ with $d(y, f(z)) \leq A$. Thus $d\left(z^{\prime}, y\right) \leq 2 A$ and so

$$
d_{B^{\prime}}\left(b^{\prime}, \hat{f}(b)\right) \leq d_{B^{\prime}}\left(p^{\prime}\left(z^{\prime}\right), p^{\prime}(y)\right)+2 E \leq 2 K A+A+2 E .
$$

Thus $\hat{f}$ is indeed a quasi-isometry. 
Definition 3.7. Let $p: X \rightarrow B$ be a coarse bundle, let $W$ be a bounded geometry quasi-geodesic metric space, and let $\lambda: W \rightarrow B$ be a coarse embedding. Then the pullback bundle is a coarse bundle $p_{W}: X_{W} \rightarrow W$ equipped with a coarse embedding $\widehat{\lambda}: X_{W} \rightarrow X$ and a constant $R \geq 0$ such that for every $w \in W, d_{\text {Haus }}\left(\widehat{\lambda}\left(D_{w}\right), D_{\lambda(w)}\right) \leq R$.

Proposition 3.8. Let $p: X \rightarrow B, W$ and $\lambda$ be as above. Then the pullback bundle $p_{W}: X_{W} \rightarrow W$ always exists and is unique up to quasi-isometry

To prove this, we require the following lemma.

Lemma 3.9. Suppose $p: X \rightarrow B$ is a $(K, A, E)$-coarse bundle and $\lambda$ : $W \rightarrow B$ is a coarse embedding. Then there exists a quasi-geodesic metric space $X_{W}$ and a coarse embedding $\hat{\lambda}: X_{W} \rightarrow B$ such that

$$
d_{\text {Haus }}\left(p^{-1}\left(N_{E}(\operatorname{im}(\lambda))\right), \operatorname{im}(\hat{\lambda})\right)<\infty \text {. }
$$

Proof. We recall that a subset of a metric space is said to be coarsely embedded if it satisfies one of the conditions of Lemma 2.7. By Lemma 2.7, it is sufficient to show that $Y:=p^{-1}\left(N_{E}(\operatorname{im}(\lambda))\right)$ is coarsely embedded.

It follows from Lemma 2.7 and Definition 3.1 that there exists a $t>0$ and a proper non-decreasing function $\eta: \mathbb{R}_{\geq 0} \rightarrow \mathbb{R}_{\geq 0}$ such that for each $b \in B$, every $x, y \in D_{b}$ can be joined by a $t$-chain in $D_{b}$ of length at most $\eta(d(x, y))$. Since $\operatorname{im}(\lambda)$ is coarsely embedded, we can take $t$ and $\eta$ sufficiently large so that every $x, y \in \operatorname{im}(\lambda)$ can be joined by a $t$-chain in $\operatorname{im}(\lambda)$ of length at most $\eta(d(x, y))$.

Suppose that $p: X \rightarrow B$ is a $(K, A, E)$-coarse bundle. Pick $x, y \in Y$ and $b_{x}, b_{y} \in \operatorname{im}(\lambda)$ such that $d\left(b_{x}, p(x)\right), d\left(b_{y}, p(y)\right) \leq E$. Thus $d_{B}\left(b_{x}, b_{y}\right) \leq$ $K d(x, y)+A+2 E$, and so $b_{x}$ and $b_{y}$ can be joined by a $t$-chain $\operatorname{in} \operatorname{im}(\lambda)$ of length at most $\eta(K d(x, y)+A+2 E)=: M$. Notice that if $d\left(b, b^{\prime}\right) \leq t$, then $d_{\text {Haus }}\left(D_{b}, D_{b^{\prime}}\right) \leq K t+A$. We can thus "lift" this $t$-chain in $\operatorname{im}(\lambda)$ to a $(K t+A)$-chain in $Y$ from $x$ to some $z \in D_{b_{y}}$ of length at most $M$.

Now observe that $z, y \in D_{b_{y}}$ and that $d(z, y) \leq d(x, y)+M(K t+A)$. Therefore $z$ and $y$ can be joined by a $t$-chain in $D_{b_{y}} \subseteq Y$ of length at most $\eta(d(x, y)+M(K t+A))$. Thus $x$ and $y$ can be joined by a $(K t+A)$-chain in $Y$ of length at most

$$
N:=M+\eta(d(x, y)+M(K t+A)) .
$$

The result now follows since $N$ can be expressed as a proper non-decreasing function of $d(x, y)$.

Proposition 3.8 follows from Lemma 3.9 and the following lemma:

Lemma 3.10. Let $p: X \rightarrow B$ be a $(K, A, E)$-coarse bundle, let $W$ and $X_{W}$ be quasi-geodesic metric spaces, and let $\lambda: W \rightarrow B$ and $\widehat{\lambda}: X_{W} \rightarrow X$ be coarse embeddings such that

$$
d_{\text {Haus }}\left(\operatorname{im}(\widehat{\lambda}), p^{-1}\left(N_{E}(\operatorname{im}(\lambda))\right)<\infty .\right.
$$

Then there are constants $K^{\prime} \geq 1, A^{\prime}, E^{\prime} \geq 0$ and $a\left(K^{\prime}, A^{\prime}, E^{\prime}\right)$-coarse bundle $p_{W}: X_{W} \rightarrow W$ such that

$$
d_{\text {Haus }}\left(\widehat{\lambda}\left(D_{w}\right), D_{\lambda(w)}\right) \leq A^{\prime}
$$

for every $w \in W$. 
Proof. We may choose $K \geq 1, t, A, E \geq 0$ and proper non-decreasing functions $\eta$ and $\phi$ such that:

- $\lambda: W \rightarrow B$ is an $(\eta, \phi)$-coarse embedding.

- $p: X \rightarrow B$ is a $(K, A, E)$-coarse bundle.

- Every $x, y \in W$ can be joined by a $t$-chain of length at most $K d_{W}(x, y)+$ A.

- There is a quasi-geodesic metric space $X_{W}$ and an $(\eta, \phi)$-coarse embedding $\hat{\lambda}: X_{W} \rightarrow X$ such that $d_{\text {Haus }}\left(p^{-1}\left(N_{E}(\operatorname{im}(\lambda))\right), \operatorname{im}(\hat{\lambda})\right) \leq A$. The existence of $X_{W}$ and $\hat{\lambda}$ follows from Lemma 3.9.

- Every $x, y \in X_{W}$ can be joined by a $t$-chain of length at most $K d_{X_{W}}(x, y)+A$.

We define $p_{W}: X_{W} \rightarrow W$ such that for every $x \in X_{W}$,

$$
d\left(\left(\lambda \circ p_{W}\right)(x),(p \circ \widehat{\lambda})(x)\right) \leq K A+A+E .
$$

The existence of such a $p_{W}$ follows from our choice of $\hat{\lambda}$. We show that $p_{W}: X_{W} \rightarrow W$ is a coarse pullback of $\lambda$.

We define $E^{\prime}:=\widetilde{\eta}(2 K A+2 A+2 E)$ and claim that $p_{W}: X_{W} \rightarrow W$ is a $\left(K^{\prime}, A^{\prime}, E^{\prime}\right)$-coarse bundle for some $K^{\prime} \geq 1$ and $A^{\prime} \geq 0$ to be determined. We first show that $p_{W}$ is coarse Lipschitz. For any $x, y \in X_{W}$, we have

$$
\begin{aligned}
\eta\left(d_{W}\left(p_{W}(x), p_{W}(y)\right)\right) & \leq d_{B}\left(\left(\lambda \circ p_{W}\right)(x),\left(\lambda \circ p_{W}\right)(y)\right) \\
& \leq d_{B}((p \circ \widehat{\lambda})(x),(p \circ \widehat{\lambda})(y))+2(K A+A+E) \\
& \leq K d_{X}(\widehat{\lambda}(x), \widehat{\lambda}(y))+2 K A+3 A+2 E \\
& \leq K \phi\left(d_{X_{W}}(x, y)\right)+2 K A+3 A+2 E .
\end{aligned}
$$

Thus there is a number $M$ such that $d_{W}\left(p_{W}(x), p_{W}(y)\right) \leq M$ whenever $d_{X_{W}}(x, y) \leq t$. Recall that any $x, y \in X_{W}$ can be joined by a $t$-chain of length at most $K d_{X_{W}}(x, y)+A$. Thus $p_{W}(x)$ and $p_{W}(y)$ can be joined by an $M$-chain of length $K d_{X_{W}}(x, y)+A$, and so $d_{W}\left(p_{W}(x), p_{W}(y)\right) \leq$ $M\left(K d_{X_{W}}(x, y)+A\right)$ for any $x, y \in X_{W}$, verifying that $p_{W}$ is coarse Lipschitz.

As in Definition 3.1, we define the fibres of $p_{W}: X_{W} \rightarrow W$ to be $D_{w}^{W}:=$ $p_{W}^{-1}\left(N_{E^{\prime}}(w)\right)$ for each $w \in W$. We pick $w \in W$ and observe that $\operatorname{im}\left(s_{\lambda(w)}\right) \subseteq$ $D_{\lambda(w)} \subseteq p^{-1}\left(N_{E}(\operatorname{im}(\lambda))\right)$, where $s_{\lambda(w)}$ is in Definition 3.1. We thus define a coarse embedding $s_{w}: F \rightarrow X_{W}$ such that

$$
d\left(\left(\hat{\lambda} \circ s_{w}\right)(f), s_{\lambda(w)}(f)\right) \leq A
$$

for all $f \in F$. It is straightforward to verify that for some $A^{\prime}$ sufficiently large,

$$
\operatorname{im}\left(s_{w}\right) \subseteq D_{w}^{W} \subseteq N_{A^{\prime}}\left(\operatorname{im}\left(s_{w}\right)\right)
$$

for all $w \in W$. The remainder of the proof follows easily from the definitions.

Our main examples of coarse bundles are finitely generated groups containing almost normal subgroups. Many basic facts concerning almost normal subgroups are proven in [CM14]. For instance:

Proposition 3.11. Suppose $G$ is a group and $H$ \& $G$. If $H^{\prime} \leq G$ is commensurable to $H$, then $H^{\prime}$ a $G$. 
Proof. It is sufficient to prove the lemma in the case that $H$ is a finite index subgroup of $H^{\prime}$ or vice-versa, which is proved in Lemma 3.10 of [CM14].

We frequently make use of the following characterisation of almost normal subgroups, also shown in [Vav13] and [CM14].

Proposition 3.12. Let $G$ be a finitely generated group with $H \leq G$. The following are equivalent:

(1) $H \backsim G$;

(2) for every $g \in G$, the coset $g H$ is at finite Hausdorff distance from $H$.

Proof. It is shown in [MSW11, Corollary 2.14] that two subgroups $H$ and $K$ of $G$ are commensurable if and only if they are at finite Hausdorff distance. It is easy to see that for all $g \in G, g H g^{-1}$ and $g H$ are at finite Hausdorff distance. Thus $g H$ and $H$ are at finite Hausdorff distance if and only if $H$ and $g \mathrm{Hg}^{-1}$ are commensurable.

This characterisation allows us to define the quotient space $G / H$.

Definition 3.13. Given a finitely generated group $G$ and an almost normal subgroup $H \backsim G$, the quotient space $G / H$ is the set of left cosets of $H$, equipped with the metric $d_{G / H}\left(g H, g^{\prime} H\right):=d_{\text {Haus }}\left(g H, g^{\prime} H\right)$.

The natural left action of $G$ on $G / H$ is an isometric action.

Proposition 3.14. Let $G$ be a finitely generated group with $H$ a $G$ finitely generated and let $p: G \rightarrow G / H$ be the quotient map $g \mapsto g H$. Then $G$ is a quasi-homogeneous coarse bundle over $G / H$ with fibre $H$.

Proof. We fix a generating set $S$ of $G$ and let $d$ be the corresponding word metric on $G$. We set $K:=\max _{s \in S} d_{\text {Haus }}(H, s H)$ and claim that $p: G \rightarrow$ $G / H$ is a $(K, 0,0)$-coarse bundle. We first note that $G / H$ has bounded geometry. This follows from the fact that $G$ has bounded geometry and distinct cosets are disjoint.

We use Proposition 2.6 to show that the quotient space $G / H$ is quasigeodesic. If $g H, k H \in G / H$, then there is an $h \in H$ with $d(g, k h) \leq$ $d_{\text {Haus }}(g H, k H)$. Since $S$ is a generating set, $g^{-1} k h=s_{1} \ldots s_{t}$ where $t \leq$ $d_{\text {Haus }}(g H, k H)$ and for each $i$, either $s_{i}$ or its inverse is contained in $S$. Then for every $i$

$$
d_{\text {Haus }}\left(g s_{1} \ldots s_{i} H, g s_{1} \ldots s_{i} s_{i+1} H\right)=d_{\text {Haus }}\left(H, s_{i+1} H\right) \leq K,
$$

demonstrating that $g H$ and $k H$ can be joined by a $K$-chain of length at most $d_{\text {Haus }}(g H, k H)$.

To prove (10) of Definition 3.1, we pick $g, k \in G$ and we note the above argument shows $g H$ and $k H$ can be joined by a $K$-chain in $G / H$ of length at most $d(g, k)$. Thus $d_{G / H}(p(g), p(k))=d_{\text {Haus }}(g H, k H) \leq K d(g, k)$. Condition (2) of Definition 3.1 follows easily from Lemma 2.4 and the fact that left multiplication in $(G, d)$ is isometric. The metric defined on $G / H$ ensures that (3) is automatically true. Quasi-homogeneity of the coarse bundle is also evident, since the transitive action of $G$ on itself permutes the set of left $H$-cosets. 
Another construction of the quotient space is carried out in [KM08]:

Definition 3.15. Let $G$ be a group and $H$ be an almost normal subgroup such that $G$ is finitely generated relative to $H$, i.e. there exists a finite set $S \subseteq G$ such that $S \cup H$ generates $G$. Then the rough Cayley graph is a graph with vertex set $G / H$ and edge set $\{(g H, g s H) \mid s \in S, g \in G\}$.

It is shown in KM08 that the rough Cayley graph is locally finite and is well-defined up to quasi-isometry. The main focus of KM08 is the situation in which $G$ is a totally disconnected locally compact group and $H$ is a compact open subgroup; in this case the rough Cayley graph is frequently referred to as the Cayley-Abels graph. In the case where $G$ is finitely generated, it is not hard to see that the quotient space $G / H$ is equivariantly quasi-isometric to the rough Cayley graph of $G$ with respect to $H$, see also CM14.

Lemma 3.16. Let $X$ and $B$ be discrete geodesic metric spaces and let $p$ : $X \rightarrow B$ be a $(K, A, E)$-coarse bundle. Pick a basepoint $b_{0} \in B$. Then:

(1) If $C$ is a coarse complementary component of $D_{b_{0}}$, then $C^{\prime}:=N_{E}(p(C))$ is a coarse complementary component of $b_{0}$. Moreover, $d_{\text {Haus }}\left(p^{-1}\left(C^{\prime}\right), C\right)<$ $\infty$.

(2) If $C^{\prime} \subseteq B$ is a coarse complementary component of $b_{0}$, then $C:=$ $p^{-1}\left(C^{\prime}\right)$ is a coarse complementary component of $D_{b_{0}}$.

In both cases, $C$ is deep if and only if $C^{\prime}$ is deep.

Proof. (11): As $C$ is a coarse complementary component of $D_{b_{0}}$, there is an $r$ such that $\partial C \subseteq N_{r}\left(D_{b_{0}}\right)$. Suppose $y \in \partial C^{\prime}$ with $x \in C^{\prime}$ and $d(x, y)=1$. We may choose $\tilde{x} \in C$ such that $d_{B}(p(\tilde{x}), x) \leq E$. Since $d_{B}(x, y)=1$, we see that $d_{\text {Haus }}\left(D_{x}, D_{y}\right) \leq K+A$, and so there exists some $\tilde{y} \in D_{y}$ with $d(\tilde{x}, \tilde{y}) \leq K+A$. Since $\tilde{x} \in C$ and $\tilde{y} \notin C$, we see that $\tilde{y} \in N_{K+A}(\partial C) \subseteq$ $N_{K+A+r}\left(D_{b_{0}}\right)$. Thus $d\left(y, b_{0}\right) \leq K(K+A+r)+A+2 E$ so that $C^{\prime}$ is a coarse complementary component of $b_{0}$.

(2): As $C^{\prime}$ is a coarse complementary component of $b_{0}$, there is an $r$ such that $\partial C^{\prime} \subseteq N_{r}\left(b_{0}\right)$. Suppose $y \in \partial C$ with $x \in C$ and $d(x, y)=1$. Then $p(x) \in C^{\prime}, p(y) \notin C^{\prime}$ and $d(p(x), p(y)) \leq K+A$. It follows that $d\left(p(y), b_{0}\right) \leq K+A+r$ and so $d_{\text {Haus }}\left(D_{p(y)}, D_{b_{0}}\right) \leq K(K+A+r)+A$. Thus $d\left(y, D_{b_{0}}\right) \leq K(K+A+r)+A$ so that $C$ is a coarse complementary component of $D_{b_{0}}$.

In both cases, $C$ is deep if and only if $C^{\prime}$ is.

Corollary 3.17. Suppose $X$ and $B$ are discrete geodesic metric spaces and that $p: X \rightarrow B$ is a coarse bundle. Let $b_{0} \in B$. Then $D_{b_{0}}$ coarsely $n$ separates $X$ if and only if $B$ has at least $n$ ends. In particular, if $G$ is a finitely generated group containing a finitely generated subgroup $H$ a $G$, then $\tilde{e}(G, H)=e(G / H)$.

Proof. If $D_{b_{0}}$ coarsely $n$-separates $X$, we can choose $n$ deep, coarse disjoint, coarse complementary components $C_{1}, \ldots, C_{n}$ of $D_{b_{0}}$. Lemma 3.16 ensures there is an $E$ such that each $C_{i}^{\prime}:=N_{E}\left(p\left(C_{i}\right)\right)$ is a deep coarse complementary component of $b_{0}$. Moreover, for $i \neq j$ we see that $C_{i}^{\prime}$ and $C_{j}^{\prime}$ are coarse disjoint since $C_{i}$ and $C_{j}$ are. Thus $B$ has at least $n$ ends. A similar argument 
demonstrates that $D_{b_{0}}$ coarsely $n$-separates $X$ if $B$ has at least $n$ ends. We recall from Proposition 2.12 that $H$ coarsely $n$-separates $G$ if and only $\tilde{e}(G, H) \geq n$. It follows that $\tilde{e}(G, H)=e(G / H)$.

The following corollary is essentially due to Hopf Hop44, since any connected locally finite vertex-transitive graph has exactly $0,1,2$ or infinitely many ends and $G / H$ is quasi-isometric to a vertex-transitive graph, namely the rough Cayley graph. See also [KR89] and CM14.

Corollary 3.18. Let $G$ be a finitely generated group and $H \backsim G$ be an almost normal subgroup. Then $e(G / H)=\tilde{e}(G, H)=0,1,2$ or $\infty$.

This is no longer true if we drop the assumption that $H \backsim G$, since then $\tilde{e}(G, H)$ can take any value in $\mathbb{N} \cup \infty$; see KR89. Corollary 3.18 tells us that the $e(G / H) \geq 3$ hypothesis in Theorem 1.4 is equivalent to the a priori stronger hypothesis $e(G / H)=\infty$.

We now relate coarse complementary components to group actions on trees. A minimal action of a group $G$ on a tree $T$ is one in which no proper subtree of $T$ is fixed by $G$. Given an edge $e \in E T$ with midpoint $m_{e}$, the components of $T \backslash m_{e}$ are called halfspaces based at $e$.

Lemma 3.19. Suppose $G$ is a finitely generated group which acts minimally on a tree $T$. Let $v_{0} \in V T$ be a basepoint. If $e \in E T$ is an edge with stabiliser $H$ and $\mathfrak{h}$ is a halfspace based at e, then

$$
C_{\mathfrak{h}}:=\left\{g \in G \mid g v_{0} \in \mathfrak{h}\right\}
$$

is a deep coarse complementary component of $H$.

Proof. It is a well known fact that $C_{\mathfrak{h}}$ is an $H$-almost invariant subset, see for instance [Sco78, Lemma 1.8]. Thus Proposition 2.12 tells us that $C_{\mathfrak{h}}$ is a deep coarse complementary component of $H$.

Corollary 3.20. Let $G$ be a finitely generated group and $H \backsim G$. Suppose $G$ acts minimally on a tree $T$, all of whose edge stabilisers are commensurable to $H$. Then $e(T) \leq \tilde{e}(G, H)$.

Proof. We fix a basepoint $v_{0} \in V$. If $e(T) \geq n$, we choose halfspaces $\mathfrak{h}_{1}, \mathfrak{h}_{2}, \ldots, \mathfrak{h}_{n}$ that are pairwise disjoint. Since all edge stabilisers are commensurable to $H, C_{\mathfrak{h}_{1}}, C_{\mathfrak{h}_{2}}, \ldots, C_{\mathfrak{h}_{n}}$ are a disjoint collection of deep coarse complementary components of $H$. Thus $H$ coarsely $n$-separates $G$ and so Proposition 2.12 ensures $\tilde{e}(G, H) \geq n$.

It is well-known that the quotient of a finitely presented group by a finitely generated group is finitely presented. The following proposition is a geometric analogue of this fact.

Proposition 3.21. Let $G$ be a group of type $F P_{2}$ with $H$ a $G$ finitely generated. Then $G / H$ is coarsely 1-acyclic.

Lemma 3.22. For each $r \geq 0$, there is $a t \geq r$ and a simplicial map

$$
\phi: P_{r}(G) \rightarrow P_{t}(G / H)
$$

defined by $\left[g_{0}, \ldots, g_{n}\right] \mapsto\left[g_{0} H, \ldots, g_{n} H\right]$. 
Proof. By Proposition 3.14, the quotient map $p: G \rightarrow G / H$ is a $(K, A, E)$ coarse bundle for some $K, A$ and $E$. Thus whenever $\left[g_{0}, \ldots, g_{n}\right]$ is a simplex of $P_{r}(G)$,

$$
d_{\text {Haus }}\left(g_{i} H, g_{j} H\right) \leq K d\left(g_{i}, g_{j}\right)+A \leq K r+A
$$

for all $i, j$, and so $\left[g_{0} H, \ldots, g_{n} H\right]$ is a simplex of $P_{K r+A}(G / H)$.

Proof of Proposition [3.21. By Theorem 2.15] and Lemma 2.16, we can pick $r$ sufficiently large such that $P_{r}(H)$ and $P_{r}(G / H)$ are connected and $P_{r}(G)$ is 1 -acyclic. We pick $t \geq r$ so that Lemma 3.22 holds. It is sufficient to show that every loop in $P_{r}(G / H)$ of the form

$$
\sigma=\left(x_{0} H, x_{1} H, \ldots, x_{n-1} H, x_{0} H\right)
$$

is the boundary of some 2-chain in $P_{t}(G / H)$. We do this by lifting $\sigma$ to a cycle $\tilde{\sigma}$ in $P_{r}(G)$. We set $\tilde{x}_{0}:=x_{0}$. We inductively define $\tilde{x}_{i}$ as follows: since $d_{\text {Haus }}\left(x_{i-1} H, x_{i} H\right) \leq r$, there is an $\tilde{x}_{i} \in x_{i} H$ with $d\left(\tilde{x}_{i-1}, \tilde{x}_{i}\right) \leq r$. We thus get a path $\tilde{x}_{0}, \ldots, \tilde{x}_{n}$ in $P_{r}(G)$, where $\tilde{x}_{0} H=\tilde{x}_{n} H=x_{0} H$. Since $P_{r}(H)$ is connected, we choose a path $\left(\tilde{x}_{n}, \tilde{x}_{n+1}, \ldots, \tilde{x}_{m}=\tilde{x}_{0}\right)$ in $P_{r}\left(x_{0} H\right)$. Thus $\tilde{\sigma}:=\left(\tilde{x}_{0}, \ldots, \tilde{x}_{m}\right)$ is a loop in $P_{r}(G)$ such that $\phi_{\#}(\tilde{\sigma})=\sigma$, with $\phi$ as in Lemma 3.22. By assumption, there is an $\omega \in C_{2}\left(P_{r}(G)\right)$ such that $\partial \omega=\tilde{\sigma}$, and hence $\partial \phi_{\#}(\omega)=\phi_{\#}(\tilde{\sigma})=\sigma$ as required.

We can now prove Theorem 3.24, which is a relative version of Dunwoody accessibility. We recall the graph theoretic interpretation of Dunwoody accessibility, first noted in MSW03. See also [DK18].

Theorem 3.23 ([Dun85]). Let X be a 2-dimensional, locally finite, 1-acyclic simplicial complex with $\operatorname{Aut}(X)$ acting cocompactly on $X$. There exists a collection of disjoint compact tracks $\left\{\tau_{i}\right\}$ that is $\operatorname{Aut}(X)$-invariant, has finitely many $\operatorname{Aut}(X)$ orbits, and every component of $X \backslash \cup_{i} \tau_{i}$ has at most one end.

Theorem 3.24. If $G$ is finitely presented and $H \backsim G$ is finitely generated, then $G$ is the fundamental group of a finite graph of groups such that:

(1) every edge group is commensurable to $H$;

(2) every vertex group is finitely generated and doesn't split over a subgroup commensurable to $H$.

Proof. As in Remark 2.9, we can remetrise $G / H$ to be a discrete geodesic metric space, which we denote $Y$. It follows from Proposition 3.21 that $Y$ is a coarsely 1-acyclic. Thus Lemma 2.16 ensures that $P_{s}(Y)$ is 1-acyclic for some sufficiently large $s$. Let $X:=P_{s}(Y)^{(2)}$. By applying Theorem 3.23 to $X$, we see that there exists a collection of finite disjoint tracks $\left\{\tau_{i}\right\}$, containing finitely many $G$-orbits, such that every component of $X \backslash \cup\left\{\tau_{i}\right\}$ has at most 1 end.

Let $T$ be the dual tree to $\left\{\tau_{i}\right\}$. By subdividing edges if necessary, we may assume that $G \curvearrowright T$ without edge inversions. We claim that the stabiliser of a track $\tau_{i}$, hence an edge of $T$, is commensurable to $H$. Indeed, let $C \subseteq X^{(0)}$ be the set of finitely many vertices that are contained in a simplex which intersects $\tau$. The pointwise stabiliser of $C$ is commensurable to $H$ and stabilises $\tau_{i}$. Moreover, the setwise stabiliser of $C$ contains the stabiliser of $\tau_{i}$ and is contained in finitely many left $H$-cosets as the track $\tau_{i}$ is finite and $X$ is locally finite. Thus the stabiliser of $\tau_{i}$ is commensurable to $H$. 
Suppose $C$ is a component of $X \backslash \cup\left\{\tau_{i}\right\}$ corresponding to some $v \in V T$. Let $X_{v}$ denote the subcomplex of $X$ consisting of the closure of cells that intersect $C$. As $X_{v}$ is connected and $G_{v}$ acts cocompactly on $X_{v}$ with cell stabilisers commensurable to $H$, hence finitely generated, it follows that $G_{v}$ is finitely generated.

Note that $C$ has finite Hausdorff distance from $Y_{v}:=\left\{g H \mid g \in G_{v}\right\} \subseteq X$. Observe that the vertex stabiliser $G_{v}$ contains some adjacent edge group, i.e. a subgroup commensurable to $H$. Thus $H_{v}:=H \cap G_{v}$ is a finite index subgroup of $H$ so that $H_{v} \backsim G_{v}$. The map $\phi: G / H_{v} \rightarrow G / H=X^{(0)}$ defined by $g H_{v} \mapsto g H$ is a quasi-isometry. Now observe that $\phi^{-1}\left(Y_{v}\right)=\left\{g H_{v} \mid g \in\right.$ $\left.G_{v}\right\}$. Since $C$ has at most one end, the quotient space $G_{v} / H_{v}$ has at most one end, hence by Corollaries 3.17 and 3.20, $G_{v}$ cannot split over any subgroup commensurable to $H$.

\section{QI RIGIDITY OF GROUPS CONTAINING ALMOST NORMAL SUBGROUPS}

We are now in a position to prove Theorem 1.4. We actually prove a more general theorem.

Theorem 4.1. Let $p: X \rightarrow B$ be a quasi-homogeneous coarse fibre bundle with fibre $F$ such that:

(1) $X$ is a coarsely uniformly n-acyclic metric space;

(2) $B$ has at least three ends;

(3) $F$ is a coarse $P D_{n}$ space.

Suppose $G$ is a finitely generated group quasi-isometric to $X$ and that $K \geq 1$ and $A \geq 0$. Then there is a constant $C$ and a subgroup $H$ \& $G$ such that the following holds: for every $(K, A)$-quasi-isometry $f: G \rightarrow X$ and $g \in G$,

for some $b \in B$.

$$
d_{\text {Haus }}\left(f(g H), D_{b}\right) \leq C
$$

To simplify the argument, we only prove Theorem 4.1 in the case where $X$ is coarsely uniformly $n$-connected. This is all that is required if one wishes to prove Theorem 1.4. The proof is almost identical when $X$ is not necessarily coarsely uniformly $n$-connected, except that we need to use the theory of metric complexes rather than metric cell complexes (see Remark 2.19).

Combining Proposition 2.10 and Lemma 3.6 with Theorem 4.1 gives the following:

Corollary 4.2. Let $X, F, B, G, H$ be as in Theorem 4.1. Then $H$ is quasiisometric to $F$ and $G / H$ is quasi-isometric to $B$.

We explain how to deduce Theorem 1.4 from Theorem 4.1 ;

Proof of Theorem 1.4. Suppose that $G$ is a group of type $F_{n+1}$ containing an almost normal coarse $P D_{n}$ subgroup $H$ with $e(G / H) \geq 3$. Then Theorem 2.15 tells us that $G$ is coarsely uniformly $n$-connected and Proposition 3.14 ensures that $p: G \rightarrow G / H$ is a quasi-homogeneous coarse bundle. Thus $p: G \rightarrow G / H$ satisfies the hypotheses of Theorem 4.1. Using Theorem 4.1 and Corollary 4.2, we see that if $G^{\prime}$ is a finitely generated group quasiisometric to $G$, it contains an almost normal subgroup $H^{\prime}$ such that $H$ is 
quasi-isometric to $H^{\prime}$ and $G / H$ is quasi-isometric to $G^{\prime} / H^{\prime}$. In particular, $H^{\prime}$ is coarse $P D_{n}$ as required.

For the remainder of this section, we fix a coarse bundle $p: X \rightarrow B$ as in Theorem 4.1. The following proposition allows us to apply the coarse topological methods of Mar18. We fix some $b_{0} \in B$ and let $D_{b_{0}} \subseteq X$ be the corresponding fibre, i.e. $p^{-1}\left(N_{E}\left(b_{0}\right)\right)$ for $E$ sufficiently large.

Proposition 4.3. Every deep coarse complementary component of $D_{b_{0}}$ is essential.

Proof. Let $C$ be a deep coarse complementary component of $D_{b_{0}}$. Lemma 3.16, ensures $C^{\prime}:=N_{E}(p(C))$ is a deep coarse complementary component of $b_{0}$ for some $E$. By Proposition 2.6, we can pick $r$ sufficiently large so that $P_{r}^{1}(B)$ is connected and the inclusion $B \rightarrow P_{r}^{1}(B)$ is a quasi-isometry. By the Arzelà-Ascoli theorem and quasi-homogeneity, there is a bi-infinite geodesic $l$ in $P_{r}^{1}(B)$ such that $l \cap C^{\prime}$ contains the vertex set of a ray $r$ of $l$. Since $\phi: l \rightarrow B$ is a coarse embedding, we use Proposition 3.8 to deduce the existence of a pullback bundle $p_{l}: X_{l} \rightarrow l$ with coarse embedding $\hat{\phi}: X_{l} \rightarrow X$.

Theorem 11.3 of KK05 now tells us that $X_{l}$ is a coarse $P D_{n+1}$ space. Since being a coarse $P D_{n+1}$ space is invariant under coarse equivalence (see [Mar18, Remark 4.4]), it follows that $\operatorname{im}(\widehat{\phi})$ is a coarse $P D_{n+1}$ space. As $l \cap C^{\prime}$ contains a ray $r$, it follows that $\operatorname{im}(\widehat{\phi}) \cap C$ contains a coarse $P D_{n+1}$ half-space whose boundary has finite Hausdorff distance from $D_{b_{0}}$. Thus Lemma 2.26 ensures that $C$ is essential.

Since $B$ has at least three ends, we use Lemma 3.16 and Proposition 4.3 to deduce the following:

Corollary 4.4. The fibre $D_{b_{0}}$ coarsely separates $X$ into three essential coarse complementary components.

Definition 4.5. A collection $\mathcal{W}$ of subsets of $X$ is said to be a uniform $P D_{n}$-set if there exist constants $K \geq 1$ and $A \geq 0$ such that the following holds: for every $W \in \mathcal{W}$, there is a $(K, A)$-quasi-isometry $f_{W}: X \rightarrow X$ with $d_{\text {Haus }}\left(f_{W}(W), D_{b_{0}}\right) \leq A$.

The set $\mathcal{W}:=\left\{D_{b} \mid b \in B\right\}$ is a uniform $P D_{n}$-set because the coarse fibre bundle $p: X \rightarrow B$ is quasi-homogeneous. Our motivation for the definition of uniform $P D_{n}$-sets is the following lemma:

Lemma 4.6. Let $\mathcal{W}$ be a uniform $P D_{n}$-set. Then there is a constant $R$ such that for every $W \in \mathcal{W}$, there is a $b \in B$ such that $d_{\text {Haus }}\left(D_{b}, W\right) \leq R$.

We can use this lemma to prove Theorem 4.1

Proof of Theorem 4.1. Let $X$ and $G$ be as in Theorem 4.1. We fix a quasiisometry $f_{0}: X \rightarrow G$. Then Corollary 4.4 ensures that $f_{0}\left(D_{b_{0}}\right)$ is coarse $P D_{n}$ subset of $G$ that coarsely separates $G$ into at least three essential coarse complementary components. Theorem 2.27 ensures that $f_{0}\left(D_{b_{0}}\right)$ has finite Hausdorff distance from a subgroup $H \leq G$. We fix $K \geq 1$ and $A \geq 0$ and let $\mathcal{F}$ be the set of all $(K, A)$-quasi-isometries from $G$ to $X$. Let $\mathcal{W}:=\{f(H) \mid f \in \mathcal{F}\}$, which is clearly a uniform $P D_{n}$-set. Since left 
multiplication in $G$ is isometric, we see that $\mathcal{W}=\{f(g H) \mid f \in \mathcal{F}, g \in G\}$. It follows from Lemma 4.6 that there is constant $R$ such that for every $f \in \mathcal{F}$ and $g \in G$, there is a $b \in B$ with $d_{\text {Haus }}\left(D_{b}, f(g H)\right) \leq R$ as required. Finally, since $d_{\text {Haus }}\left(D_{b}, D_{b^{\prime}}\right)<\infty$ for any $b, b^{\prime} \in B$, we see that $d_{\text {Haus }}(H, g H)<\infty$ for every $g \in G$, and so Proposition 3.12 ensures $H$ a $G$.

We now prove Lemma 4.6. As $X$ is coarsely uniformly $n$-connected, we fix some uniformly $n$-connected metric cell complex $(X, \mathbf{X})$.

Lemma 4.7. Let $\mathcal{W}$ be a uniform $P D_{n}$-set. Then there exist constants $D$ and $E$ such that:

(1) For every $W \in \mathcal{W}$, there is a decomposition of $X \backslash N_{E}(W)=C_{1}^{W} \cup$ $C_{2}^{W} \cup C_{3}^{W}$ into disjoint coarse complementary components of $W$ with $\partial C_{i}^{W} \subseteq N_{E}(W)$ for $i=1,2,3$.

(2) There are distinct cohomology classes $\left[\alpha_{1}^{W}\right],\left[\alpha_{2}^{W}\right] \in \operatorname{ker}\left(H_{c}^{n+1}(\mathbf{X}) \rightarrow\right.$ $\left.H^{n+1}(\mathbf{X})\right)$ such that for $i=1,2$ and every $x \in W,\left[\alpha_{i}^{W}\right]$ can be represented by a cocycle supported in $N_{D}(x)$.

(3) For $i=1,2$, the cohomology class $\left[\alpha_{i}^{W}\right]$ can be represented by a cocycle supported in $C_{i}^{W} \backslash N_{E}(W)$.

(4) A cohomology class $[\omega] \in \operatorname{ker}\left(H_{c}^{n+1}(\mathbf{X}) \rightarrow H^{n+1}(\mathbf{X})\right)$ is represented by cocycles supported in any two of $C_{1}^{W} \backslash N_{E}(W), C_{2}^{W} \backslash N_{E}(W)$ and $C_{3}^{W} \backslash N_{E}(W)$ only if $[\omega]=[0]$.

Proof. We let $\left\{f_{W} \mid W \in \mathcal{W}\right\}$ be quasi-isometries as in Definition 4.5. We recall from Corollary 4.4 that $D_{b_{0}}$ coarsely separates $X$ into at least three essential components. Thus there exist essential coarse disjoint, coarse complementary components $C_{1}, C_{2}$ and $C_{3}$ of $D_{b_{0}}$, where we may assume without loss of generality that $C_{3}=X \backslash\left(C_{1} \cup C_{2}\right)$. By taking $E$ sufficiently large, we can easily deduce (11) by taking $C_{i}^{W}:=f_{W}^{-1}\left(C_{i}\right) \backslash N_{E}(W)$ for each $i \in\{1,2,3\}$ and $W \in \mathcal{W}$.

Parts (2)-(4) follow from Lemma 2.28 and the fact that each quasi-isometry $f_{W}$ induces a uniformly proper chain homotopy equivalence on $C_{\bullet}\left(\mathbf{X}^{(n+1)}\right)$, whose constants are independent of $W \in \mathcal{W}$. For instance, see Theorem 2.7 of [MSW11, Proposition 9.48 of [DK18] or Lemma 3.21 of [Mar18].

Our first step in proving Lemma 4.6 is the following:

Lemma 4.8. For any quasi-isometry $f: X \rightarrow X, d_{\text {Haus }}\left(f\left(D_{b_{0}}\right), D_{b_{0}}\right)<\infty$.

Proof. We observe that $\mathcal{W}:=\cup\left\{D_{b} \mid b \in B\right\} \cup \cup\left\{f\left(D_{b}\right) \mid b \in B\right\}$ is a uniform $P D_{n}$-set, so we may apply Lemma 4.7. We thus choose $D, E, C_{i}^{W}$ and $\alpha_{i}^{W}$ so the conclusions of Lemma 4.7 hold. We suppose that $f$ is a $(K, A)$-quasi-isometry and set $C_{i}:=C_{i}^{D_{b_{0}}}$ for $i=1,2$ and 3. Since each $C_{i}$ is deep, we choose $x_{i} \in C_{i}$ such that $d\left(x_{i}, D_{b_{0}}\right)>D+E+A$, and a $y_{i} \in X$ such that $d\left(f\left(y_{i}\right), x_{i}\right) \leq A$. Let $b_{i}=p\left(y_{i}\right)$ for $i=1,2$.

Since $f\left(D_{b_{1}}\right), f\left(D_{b_{2}}\right) \in \mathcal{W}$, Lemma 4.7 ensures that there exist non-zero cohomology classes $\left[\alpha_{1}\right],\left[\alpha_{2}\right] \in \operatorname{ker}\left(H_{c}^{n+1}(\mathbf{X}) \rightarrow H^{n+1}(\mathbf{X})\right)$ such that for every $z_{i} \in f\left(D_{b_{i}}\right),\left[\alpha_{i}\right]$ can be represented by a cocycle supported in $N_{D}\left(z_{i}\right)$. As $f\left(y_{i}\right) \in C_{i} \backslash N_{D+E}\left(D_{b_{0}}\right)$, we see that each $\left[\alpha_{i}\right]$ can be represented by a cocycle supported in $C_{i} \backslash N_{E}\left(D_{b_{0}}\right)$ for $i=1,2$. Thus

$$
f\left(D_{b_{i}}\right) \subseteq C_{i} \cup N_{D+E}\left(D_{b_{0}}\right),
$$


otherwise $\left[\alpha_{i}\right]$ could be represented by cocycles supported in at least two of $C_{1} \backslash N_{E}\left(D_{b_{0}}\right), C_{2} \backslash N_{E}\left(D_{b_{0}}\right)$ and $C_{3} \backslash N_{E}\left(D_{b_{0}}\right)$, which would contradict Lemma 4.7 .

Since $f\left(D_{b_{1}}\right)$ and $f\left(D_{b_{2}}\right)$ are at finite Hausdorff distance, we pick $r$ sufficiently large so that $f\left(D_{b_{2}}\right) \subseteq N_{r}\left(f\left(D_{b_{1}}\right)\right)$. Since $N_{r}\left(f\left(D_{b_{1}}\right)\right) \subseteq C_{1} \cup$ $N_{r+D+E}\left(D_{b_{0}}\right)$, we see that

$$
\begin{aligned}
f\left(D_{b_{2}}\right) & =f\left(D_{b_{2}}\right) \cap N_{r}\left(f\left(D_{b_{1}}\right)\right) \\
& \subseteq\left(C_{2} \cup N_{D+E}\left(D_{b_{0}}\right)\right) \cap\left(C_{1} \cup N_{r+D+E}\left(D_{b_{0}}\right)\right) \subseteq N_{r+D+E}\left(D_{b_{0}}\right) .
\end{aligned}
$$

Since $d_{\text {Haus }}\left(f\left(D_{b_{2}}\right), f\left(D_{b_{0}}\right)\right)<\infty$, it follows from Theorem 2.24 that $f\left(D_{b_{0}}\right)$ and $D_{b_{0}}$ are at finite Hausdorff distance.

Lemma 4.9. Let $\mathcal{W}, D$ and $E$ be as in Lemma 4.7. Suppose that

(1) $D+E<d_{\text {Haus }}\left(W, W^{\prime}\right)<\infty$ for all distinct $W, W^{\prime} \in \mathcal{W}$;

(2) there exist $C \geq 0$ and $x_{0} \in X$ such that $d\left(x_{0}, W\right) \leq C$ for all $W \in \mathcal{W}$.

Then $\mathcal{W}$ is finite.

Proof. Let $\left\{\left[\alpha_{i}^{W}\right]\right\}_{W \in \mathcal{W}, i=1,2}$ be the cohomology classes that satisfy the properties of Lemma 4.7. Each cohomology class $\left[\alpha_{i}^{W}\right]$ can be represented by a cocycle supported in $N_{C+D}\left(x_{0}\right)$, thus there are only finitely many such classes. To show $\mathcal{W}$ is finite, it is sufficient to show that if $W$ and $W^{\prime}$ are distinct elements of $\mathcal{W}$, then $\left\{\left[\alpha_{1}^{W}\right],\left[\alpha_{2}^{W}\right]\right\} \neq\left\{\left[\alpha_{1}^{W^{\prime}}\right],\left[\alpha_{2}^{W^{\prime}}\right]\right\}$. Indeed, if $W \neq W^{\prime}$, then by reversing the roles of $W$ and $W^{\prime}$ if necessary we can choose $x \in W$ such that $d\left(x, W^{\prime}\right)>D+E$. Hence both $\left[\alpha_{1}^{W}\right]$ and $\left[\alpha_{2}^{W}\right]$ can be represented by cocycles supported in $C_{i}^{W^{\prime}} \backslash N_{E}\left(W^{\prime}\right)$ for some $i \in\{1,2,3\}$. This ensures that $\left\{\left[\alpha_{1}^{W}\right],\left[\alpha_{2}^{W}\right]\right\} \neq\left\{\left[\alpha_{1}^{W^{\prime}}\right],\left[\alpha_{2}^{W^{\prime}}\right]\right\}$.

Proof of Lemma 4.6. The following proof is based on the argument used in the proof of Vav13, Proposition 4.3], although in a more general context. We fix $x_{0}, K, A,\left\{f_{x}\right\}_{x \in X}$ and $\left\{\overline{f_{x}}\right\}_{x \in X}$ as in Lemma 3.5. We set $D$ and $E$ as in Lemma 4.7 .

We assume for contradiction that no such $R$ exists, and hence $\mathcal{W}$ is necessarily infinite. It follows from Lemma 4.8 that $d_{\text {Haus }}\left(D_{b_{0}}, W\right)<\infty$ for every $W \in \mathcal{W}$. We can thus take a sequence $\left(W_{i}\right)$ in $\mathcal{W}$ such that $\min _{b \in B} d_{\text {Haus }}\left(D_{b}, W_{i}\right)$ is unbounded. Choose $b_{i} \in B$ such that

$$
\min _{b \in B} d_{\text {Haus }}\left(D_{b}, W_{i}\right)=d_{\text {Haus }}\left(D_{b_{i}}, W_{i}\right) .
$$

By passing to a subsequence, we may suppose that

$$
d_{\text {Haus }}\left(D_{b_{j}}, W_{j}\right)>K^{2} d_{\text {Haus }}\left(W_{i}, D_{b_{i}}\right)+K(2 A+D+E)+3 A
$$

for all $j>i$.

We claim that $d_{\text {Haus }}\left(f_{x}\left(W_{i}\right), f_{x^{\prime}}\left(W_{j}\right)\right)>D+E$ for all $i \neq j$ and $x, x^{\prime} \in X$. We assume for contradiction that $d_{\text {Haus }}\left(f_{x}\left(W_{i}\right), f_{x^{\prime}}\left(W_{j}\right)\right) \leq D+E$ for some $i \neq j$ and $x, x^{\prime} \in X$. Without loss of generality, we may suppose $j>i$.

\footnotetext{
${ }^{1}$ Recall cohomology is taken with $\mathbb{Z}_{2}$ coefficients.
} 
Since $f_{x}$ is $A$-fibre preserving, there is a $b \in B$ with $d_{\text {Haus }}\left(f_{x}\left(D_{b_{i}}\right), D_{b}\right) \leq$ A. Thus

$$
\begin{aligned}
d_{\text {Haus }}\left(f_{x^{\prime}}\left(W_{j}\right), D_{b}\right) & \leq d_{\text {Haus }}\left(f_{x^{\prime}}\left(W_{j}\right), f_{x}\left(D_{b_{i}}\right)\right)+A \\
& \leq d_{\text {Haus }}\left(f_{x^{\prime}}\left(W_{j}\right), f_{x}\left(W_{i}\right)\right)+d_{\text {Haus }}\left(f_{x}\left(W_{i}\right), f_{x}\left(D_{b_{i}}\right)\right)+A \\
& \leq K d_{\text {Haus }}\left(W_{i}, D_{b_{i}}\right)+2 A+D+E .
\end{aligned}
$$

Since $\overline{f_{x^{\prime}}}$ is also $A$-preserving, there is a $b^{\prime} \in B$ with $d_{\text {Haus }}\left(\bar{f}_{x^{\prime}}\left(D_{b}\right), D_{b^{\prime}}\right) \leq A$. Thus as $\bar{f}_{x^{\prime}} \circ f_{x^{\prime}}$ is $A$-close to the identity, we see

$$
\begin{aligned}
d_{\text {Haus }}\left(W_{j}, D_{b^{\prime}}\right) & \leq d_{\text {Haus }}\left(\overline{f_{x^{\prime}}}\left(f_{x^{\prime}}\left(W_{j}\right)\right), \overline{f_{x^{\prime}}}\left(D_{b}\right)\right)+2 A \\
& \leq K d_{\text {Haus }}\left(f_{x^{\prime}}\left(W_{j}\right), D_{b}\right)+3 A \\
& \leq K^{2} d_{\text {Haus }}\left(W_{i}, D_{b_{i}}\right)+K(2 A+D+E)+3 A
\end{aligned}
$$

It follows from the definition of $b_{j}$ that

$d_{\text {Haus }}\left(W_{j}, D_{b_{j}}\right) \leq d_{\text {Haus }}\left(W_{j}, D_{b^{\prime}}\right) \leq K^{2} d_{\text {Haus }}\left(W_{i}, D_{b_{i}}\right)+K(2 A+D+E)+3 A$, contradicting (1) and so proving the claim.

For each $W_{i}$, we pick an arbitrary $x_{i} \in W_{i}$ and set $\widehat{W}_{i}:=f_{x_{i}}\left(W_{i}\right)$ so that $d\left(x_{0}, \widehat{W}_{i}\right) \leq A$. It follows from the above claim that $d_{\text {Haus }}\left(\widehat{W}_{i}, \widehat{W}_{j}\right)>D+E$ for all $i \neq j$. Thus $\left\{\widehat{W}_{i} \mid i \in \mathbb{N}\right\}$ is an infinite uniform $P D_{n}$-set that contradicts Lemma 4.9.

We explain how to reformulate Theorem 1.4 in terms of graphs of groups:

Corollary 4.10. Let $G$ be a group of type $F_{n+1}$. Suppose that $G$ is the fundamental group of a graph of groups $\mathcal{G}$ with the following properties:

(1) the associated Bass-Serre tree has at least three ends;

(2) all conjugates of all edge groups are coarse $P D_{n}$ groups and are commensurable to one another;

(3) each vertex group is finitely generated and doesn't split over a subgroup commensurable to one of its incident edge groups.

If $G^{\prime}$ is a finitely generated group quasi-isometric to $G$, it is also the fundamental group of a graph of groups satisfying (1)-(3).

Proof. We claim a finitely presented group has an almost normal coarse $P D_{n}$ subgroup $H$ with $e(G / H) \geq 3$ if and only if $G$ splits as a graph of groups satisfying (1)-(3). Corollary 4.10 now follows from Theorem 1.4 and the above claim. If $G$ splits as a graph of groups satisfying (1)-(3), let $H$ be any edge group. Since all conjugates of $H$ are commensurable to $H$, we deduce that $H \backsim G$. Corollaries 3.17 and 3.20 then ensure that $e(G / H) \geq 3$.

Conversely, suppose that $G$ contains an almost normal, coarse $P D_{n}$ subgroup $H$ with $e(G / H) \geq 3$. Theorem 3.24 ensures that $G$ splits as a graph of groups satisfying (21) and (3). It is clear from the proof of Theorem 3.24 that as $e(G / H) \geq 3$, the corresponding Bass-Serre tree has at least three ends.

We now prove a partial quasi-isometric classification for such groups. We closely follow the proof of [PW02, Theorem 3.1]. 
Theorem 4.11. Let $G$ and $G^{\prime}$ be groups of type $F_{n+1}$ that are fundamental groups of finite graphs of groups $\mathcal{G}$ and $\mathcal{G}^{\prime}$ satisfying conditions (1)-(3) of Corollary 4.10. Suppose that $G$ and $G^{\prime}$ are quasi-isometric. Then for each essential vertex group $G_{v}$ of $\mathcal{G}$, there is an essential vertex group $G_{v^{\prime}}^{\prime}$ of $\mathcal{G}^{\prime}$ and a fibre-preserving quasi-isometry $G_{v} \rightarrow G_{v^{\prime}}^{\prime}$. Conversely, for each essential vertex $G_{v^{\prime}}^{\prime}$ of $\mathcal{G}^{\prime}$ there exists an essential vertex group $G_{v}$ of $\mathcal{G}$ and a fibre-preserving quasi-isometry $G_{v} \rightarrow G_{v^{\prime}}^{\prime}$.

Proof. We recall from the proof of Theorem 3.24 that there is an infinite ended, 1-acyclic 2-complex $X$ on which $G$ acts cocompactly. There exists a collection of finitely many $G$-orbits of finite disjoint tracks $\left\{\tau_{i}\right\}_{i \in I}$ such that each component of $X \backslash \cup_{i \in I}\left\{\tau_{i}\right\}$ is either compact or 1-ended. The dual tree $T$ gives the Bass-Serre tree of the graph of groups in Theorem 3.24. Similarly, let $X^{\prime}$ be the corresponding complex for $G^{\prime}$, let $\left\{\tau_{j}\right\}_{j \in J}$ be the corresponding collection of tracks, and let $T^{\prime}$ be the dual tree.

Suppose $f: G \rightarrow G^{\prime}$ is a quasi-isometry. By Theorem $4.1 f$ is fibrepreserving, so Lemma 3.6 ensures $f$ induces a quasi-isometry $\hat{f}: X \rightarrow X^{\prime}$. It follows from the proof of [PW02, Theorem 3.1] that $\hat{f}$ sends 1-ended components of $X \backslash \cup\left\{\tau_{i}\right\}$ to a uniform metric neighbourhood of a 1-ended component of $X^{\prime} \backslash \cup\left\{\tau_{j}\right\}$. It was shown in the proof of Theorem 3.24 that these components are quasi-isometric to quotient spaces of the form $G_{v} / H_{v}$ or $G_{v^{\prime}}^{\prime} / H_{v^{\prime}}^{\prime}$. Moreover, as $d_{\text {Haus }}(\hat{f}(g H), f(g H)) \leq B$ it follows that $f$ sends each $G_{v} \in V_{\infty} \mathcal{G}$ to within uniform Hausdorff distance of some $G_{v^{\prime}}^{\prime} \in V_{\infty} \mathcal{G}^{\prime}$ and vice-versa. Proposition 2.10 then ensures $f$ induces the required quasiisometries.

\section{Fibre Distortion}

Theorem 1.4. Corollary 4.10 and Theorem 4.11 give necessary conditions for two groups in a certain class to be quasi-isometric. Can we obtain finer QI invariants that yield finer QI classification and rigidity results? For example, observe that $B S(1,3)=\left\langle a, t \mid t a t^{-1}=a^{3}\right\rangle$ and $F_{2} \times \mathbb{Z}$ have the same Bass-Serre tree in the following sense: both act on 4 -valent trees with all edge and vertex stabilisers isomorphic to $\mathbb{Z}$. Thus Corollary 4.10 and Theorem 4.11 cannot distinguish them up to quasi-isometry.

To distinguish such groups, we use the notion of height change defined in [FM99] and Why01. Let $H:=\langle a\rangle$ a $B S(1,3)$. We think of $B S(1,3)$ as fibred over its left $H$-coset space with fibres corresponding to $H$-cosets. Endowing each fibre with the path metric, the closest point projection from $H$ to $t^{k} H$ coarsely distorts distances by a factor of $3 k$; this is because $t^{k} a^{i}=$ $a^{3 i} t^{k}$. In contrast, no such distortion can occur in $F_{2} \times \mathbb{Z}$. These sort of phenomena will be used to prove Theorems 5.10 and 5.16,

We fix a finitely generated group $G$ containing a finitely generated, almost normal subgroup $H \backsim G$. For each $g \in G$, let $p_{g}: H \rightarrow g H$ be a closest point projection map, i.e. any map such that $d\left(x, p_{g}(x)\right) \leq d(x, y)$ for all $y \in g H$. We remark that by Proposition 3.12, $H$ and $g H$ are at finite Hausdorff distance. Any map $f: H \rightarrow g H$ which moves points a uniform distance will be close to $p_{g}$, i.e. if $\sup _{h \in H} d(f(h), h)<\infty$, then $\sup _{h \in H} d\left(f(h), p_{g}(h)\right)<$ $\infty$. Thus $p_{g}$ is coarsely well-defined. 
Example 5.1. Suppose $\mathbb{Z}\left[v_{1}, \ldots, v_{n}\right]=\mathbb{Z}^{n} \cong H$ a $G$. Since $g H g^{-1}$ and $H$ are commensurable, for each $v_{j}$ there exist integers $q_{j}, p_{1 j}, \ldots, p_{2 j}$ such that $v_{j}^{q_{j}}=g v_{1}^{p_{1 j}} v_{2}^{p_{2 j}} \ldots v_{n}^{p_{n j}} g^{-1}$. We associate to each $g \in G$ a matrix $A_{g} \in$ $G L(n, \mathbb{Q})$, whose $(i, j)$ entry is $\frac{p_{i j}}{q_{j}}$. Then

$$
\sup _{h \in H} d\left(p_{g}(h), g\left(\left\lfloor A_{g} \cdot h\right\rfloor\right)\right)<\infty,
$$

where $\left\lfloor\left(x_{1}, \ldots, x_{n}\right)\right\rfloor:=\left(\left\lfloor x_{1}\right\rfloor, \ldots,\left\lfloor x_{n}\right\rfloor\right)$. We show this in the case $n=1$; the general case is similar but notationally unwieldy.

We write $v_{1}=v, q_{1}=q$ and $p_{11}=p$. For each $k \in \mathbb{Z}$, let $r_{k}:=k-q\left\lfloor\frac{k}{q}\right\rfloor$. Then $v^{k}=v^{q\left\lfloor\frac{k}{q}\right\rfloor+r_{k}}=g v^{p\left\lfloor\frac{k}{q}\right\rfloor} g^{-1} v^{r_{k}}$. Since

$$
\sup _{k \in \mathbb{Z}} d\left(g v^{\left\lfloor\frac{p}{q} k\right\rfloor}, g v^{p\left\lfloor\frac{k}{q}\right\rfloor} g^{-1} v^{r_{q}}\right)<\infty,
$$

we see that $\sup _{k \in \mathbb{Z}} d\left(g v^{\left\lfloor\frac{\underline{q}}{q} k\right\rfloor}, v^{k}\right)<\infty$. We deduce from the above discussion that

$$
\sup _{k \in \mathbb{Z}} d\left(p_{g}\left(v^{k}\right), g v^{\left\lfloor\frac{p}{q} k\right\rfloor}\right)<\infty .
$$

We pick a finite generating set for $H$ and equip $H$ with the word metric $d_{H}$. For each $g \in G$, the map $H \rightarrow G$ given by $h \mapsto g h$ is a coarse embedding. This follows from Lemma 2.4 and the fact that left multiplication by $g$ is isometric. Thus one equips each coset $g H$ with the metric $d_{g H}\left(g h, g h^{\prime}\right):=$ $d_{H}\left(h, h^{\prime}\right)$. Proposition 2.10 ensures that $p_{g}: H \rightarrow g H$ is a quasi-isometry with respect to the above metrics.

Remark 5.2. If $g H=k H$, then for all $h, h^{\prime} \in H$

$$
d_{g H}\left(g h, g h^{\prime}\right)=d_{H}\left(h, h^{\prime}\right)=d_{H}\left(k^{-1} g h, k^{-1} g h^{\prime}\right)=d_{k H}\left(g h, g^{\prime} h\right) .
$$

Thus $d_{g H}$ depends only on the coset $g H$ and is independent of $g$.

Definition 5.3. Let $X$ and $Y$ be metric spaces and $f: X \rightarrow Y$ be a quasi-isometry. We define

$$
\kappa(f):=\inf \{K \geq 1 \mid f \text { is a }(K, A) \text {-quasi-isometry for some } A \geq 0\} .
$$

Let $G$ be a finitely generated group with $H \backsim G$ a finitely generated almost normal subgroup. We define a function $F: G / H \rightarrow \mathbb{R}$ by $g H \mapsto \log \left(\kappa\left(p_{g}\right)\right)$, which we call the fibre distortion function.

Example 5.4. Suppose $H \backsim G$ is a finitely generated free abelian subgroup. We note that the word metric with respect to a $\mathbb{Z}$-basis of $H$ is simply the $l_{1}$-norm of $H$. Define $A_{g}$ as in Example [5.1. Choose $B$ such that

$$
\sup _{h \in H} d\left(p_{g}(h), g\left(\left\lfloor A_{g} \cdot h\right\rfloor\right)\right) \leq B .
$$

We also observe that $\left|\left\lfloor A_{g} \cdot h\right\rfloor-A_{g} \cdot h\right|_{1} \leq n$ for all $h \in \mathbb{R}^{n}$. Thus for any $h \neq h^{\prime} \in H$,

$$
\left|\frac{d_{g H}\left(p_{g}(h), p_{g}\left(h^{\prime}\right)\right)}{d_{H}\left(h, h^{\prime}\right)}-\frac{\left|A_{g}\left(h^{\prime}-h\right)\right|_{1}}{\left|h^{\prime}-h\right|_{1}}\right| \leq \frac{2 B+2 n}{d_{H}\left(h, h^{\prime}\right)},
$$

and so

$$
K\left(h, h^{\prime}\right) d_{H}\left(h, h^{\prime}\right)-A \leq d_{g H}\left(p_{g}(h), p_{g}\left(h^{\prime}\right)\right) \leq K\left(h, h^{\prime}\right) d_{H}\left(h, h^{\prime}\right)+A .
$$



where $K\left(h, h^{\prime}\right):=\frac{\left|A_{g}\left(h^{\prime}-h\right)\right|_{1}}{\left|h^{\prime}-h\right|_{1}}$ and $A:=2 B+2 n$. Notice that

$$
\frac{1}{\left\|A_{g}\right\|_{1}} \leq K\left(h, h^{\prime}\right) \leq\left\|A_{g}\right\|_{1},
$$

where $\left\|A_{g}\right\|_{1}$ is the matrix norm of $A_{g}$. Thus $F(g H)=\left|\log \left(\left\|A_{g}\right\|_{1}\right)\right|$.

We now show that fibre distortion is a quasi-isometry invariant up to some additive error.

Proposition 5.5. Suppose that $G$ and $G^{\prime}$ are groups containing almost normal subgroups $H$ \& $G$ and $H^{\prime}$ \& $G^{\prime}$ such that the hypotheses of Theorem 1.4 are satisfied. Let $F$ and $F^{\prime}$ be the associated fibre distortion functions. If $f: G \rightarrow G^{\prime}$ is a quasi-isometry and $\widehat{f}: G / H \rightarrow G^{\prime} / H^{\prime}$ is the induced quasi-isometry as in Lemma 3.6, there is a constant $C \geq 0$ such that

$$
\left|F^{\prime}(\widehat{f}(g H))-F(g H)\right| \leq C .
$$

The existence of $\widehat{f}$ follows from Theorem 4.1, since any quasi-isometry $f: G \rightarrow G^{\prime}$ is fibre-preserving. To prove Proposition 5.5 we make use of the following lemma:

Lemma 5.6. Suppose that $f: X \rightarrow Y$ is a quasi-isometry. If $g: W \rightarrow X$ and $h: Y \rightarrow Z$ are $(K, A)$-quasi-isometries, then

$$
\left.\frac{\kappa(f)}{K^{2}} \leq \kappa(h \circ f \circ g)\right) \leq \kappa(f) K^{2} .
$$

Proof. Let $\epsilon>0$ and $M:=\kappa(f)$. Then there exists a $B \geq 0$ such that $f$ is a $(M+\epsilon, B)$-quasi-isometry. Thus $h \circ f \circ g$ is a $\left(K^{2}(M+\epsilon), B^{\prime}\right)$-quasi-isometry for some $B^{\prime}>0$. This shows that $\kappa(h \circ f \circ g) \leq K^{2} M=K^{2} \kappa(f)$.

To obtain the lower bound, we observe that as $f$ is not an $(M-\epsilon, C)$ quasi-isometry for any $C$, there exist sequences of points $\left(x_{n}\right),\left(x_{n}^{\prime}\right)$ such that for each $n$, either

$$
d\left(f\left(x_{n}\right), f\left(x_{n}^{\prime}\right)\right)>(M-\epsilon) d\left(x_{n}, x_{n}^{\prime}\right)+n
$$

or

$$
d\left(f\left(x_{n}\right), f\left(x_{n}^{\prime}\right)\right)<\frac{1}{M-\epsilon} d\left(x_{n}, x_{n}^{\prime}\right)-n .
$$

There exist sequences $\left(w_{n}\right)$ and $\left(w_{n}^{\prime}\right)$ in $W$ such that for all $n, d\left(g\left(w_{n}\right), x_{n}\right) \leq$ $A$ and $d\left(g\left(w_{n}^{\prime}\right), x_{n}^{\prime}\right) \leq A$. Thus there is a constant $B^{\prime \prime}>0$ such that for every $n$, either

or

$$
d\left((h \circ f \circ g)\left(w_{n}\right),(h \circ f \circ g)\left(w_{n}^{\prime}\right)\right)>\frac{M-\epsilon}{K^{2}} d\left(w_{n}, w_{n}^{\prime}\right)+\frac{n}{K}+B^{\prime \prime}
$$

$$
d\left((h \circ f \circ g)\left(w_{n}\right),(h \circ f \circ g)\left(w_{n}^{\prime}\right)\right)<\frac{K^{2}}{M-\epsilon} d\left(w_{n}, w_{n}^{\prime}\right)-n K-B^{\prime \prime} .
$$

By taking $n$ sufficiently large, we deduce that $h \circ f \circ g$ is not an $\left(\frac{M-\epsilon}{K^{2}}, A^{\prime}\right)$ quasi-isometry for any $A^{\prime} \geq 0$. Thus $\kappa(h \circ f \circ g) \geq \frac{M}{K^{2}}=\frac{\kappa(f)}{K^{2}}$.

Proof of Proposition 5.5. Let $f: G \rightarrow G^{\prime}$ be a $(K, A)$ quasi-isometry. By Theorem 4.1 and Lemma 3.6, there is a constant $C \geq 0$ such that:

(1) $d_{\text {Haus }}\left(f(H), H^{\prime}\right) \leq C$; 
(2) for all $g \in G$, then $d_{\text {Haus }}(f(g H), \widehat{f}(g H)) \leq C$.

We thus define $f_{0}: H \rightarrow H^{\prime}$ such that $d\left(f_{0}(h), f(h)\right) \leq C$ for all $h \in$ $H$. We fix $g \in G$, and similarly define $f_{g}: g H \rightarrow \widehat{f}(g H)$ such that $d\left(f_{g}(g h), f(g h)\right) \leq C$ for all $h \in H$. Let $p_{g}: H \rightarrow g H$ and $p_{g}^{\prime}: H^{\prime} \rightarrow \widehat{f}(g H)$ be closest point projections. By Proposition 2.10 there exist constants $K^{\prime} \geq 1$ and $A^{\prime} \geq 0$, independent of $g$, such that $f_{0}$ and $f_{g}$ are $\left(K^{\prime}, A^{\prime}\right)$ quasi-isometries for all $g \in G$. Moreover, we may choose $\left(K^{\prime}, A^{\prime}\right)$ sufficiently large so that $f_{0}$ has a quasi-inverse $\bar{f}_{0}$ that is also a $\left(K^{\prime}, A^{\prime}\right)$-quasi-isometry.

Since $\sup _{h \in H} d\left(f_{g} \circ p_{g}(h), f(h)\right)<\infty$ and $\sup _{h \in H} d\left(p_{g}^{\prime} \circ f_{0}(h), f(h)\right)<\infty$, we see that $\sup _{h \in H} d\left(p_{g}^{\prime} \circ f_{0}(h), f_{g} \circ p_{g}(h)\right)<\infty$. Thus

$$
\sup _{h^{\prime} \in H^{\prime}} d\left(p_{g}^{\prime}\left(h^{\prime}\right),\left(f_{g} \circ p_{g} \circ \bar{f}_{0}\right)\left(h^{\prime}\right)\right)<\infty .
$$

It follows from Lemma 5.6 that $\frac{\kappa\left(p_{g}\right)}{\left(K^{\prime}\right)^{2}} \leq \kappa\left(p_{g}^{\prime}\right)=\kappa\left(f_{g} \circ p_{g} \circ \bar{f}_{0}\right) \leq\left(K^{\prime}\right)^{2} \kappa\left(p_{g}\right)$ and so $\left|F^{\prime}(\widehat{f}(g H))-F(g H)\right| \leq 2 \log \left(K^{\prime}\right)$.

In the case where $H$ is infinite cyclic, we define a related invariant called the height function, generalising the height function used in Why01.

Definition 5.7. Let $G$ be a finitely generated group containing an almost normal subgroup $\mathbb{Z} \cong\langle t\rangle=H$ a $G$. Pick $q, p \in \mathbb{Z}$ such that $t^{q}=g t^{p} g^{-1}$. The height function $h: G \rightarrow \mathbb{R}$ is defined by $g \mapsto \log \left(\left|\frac{p}{q}\right|\right)$.

The height function is well-defined as the ratio $\frac{p}{q}$ depends only on $g$. The following is evident from Examples 5.1 and 5.4:

Lemma 5.8. If $H$ a $G$ is infinite cyclic, then $|h(g)|=F(g H)$.

One advantage of the height function over the fibre distortion function is the following:

Proposition 5.9. The height function $h: G \rightarrow(\mathbb{R},+)$ is a homomorphism.

Proof. Let $g, k \in G$. We pick non-zero integers $n, m, r, s$ such that $t^{m}=$ $g t^{n} g^{-1}$ and $t^{r}=k t^{s} k^{-1}$. Thus $t^{m r}=g t^{n r} g^{-1}=g k t^{n s} k^{-1} g^{-1}$ and so

$$
h(g k)=\log \left(\left|\frac{n s}{m r}\right|\right)=\log \left(\left|\frac{n}{m}\right|\right)+\log \left(\left|\frac{s}{r}\right|\right)=h(g)+h(k) .
$$

As $g^{-1} t^{m} g=t^{n}$, we see that $h\left(g^{-1}\right)=\log \left(\left|\frac{m}{n}\right|\right)=-\log \left(\left|\frac{n}{m}\right|\right)=-h(g)$. Thus $h$ is a homomorphism.

We now show quasi-isometric rigidity for finitely presented $\mathbb{Z}$-by-( $\infty$-ended) groups. This result also holds for groups of type $F P_{2}$.

Theorem 5.10. Let $G$ be a finitely presented $\mathbb{Z}-b y-(\infty$ ended) group. If $G^{\prime}$ is any finitely generated group quasi-isometric to $G$, it is also $\mathbb{Z}-b y-(\infty$ ended). Moreover, any quasi-isometry $f: G \rightarrow G^{\prime}$ induces a quasi-isometry $G / \mathbb{Z} \rightarrow G^{\prime} / \mathbb{Z}$ between quotient groups.

Proof. Suppose $G$ is a finitely presented group that contains an infinite cyclic normal subgroup $H$, with $G / H$ infinite ended. As $H \triangleleft G$, the height function $h: G \rightarrow \mathbb{R}$ is zero. Thus the fibre distortion function $F$ is zero by Lemma 5.8. Suppose $G^{\prime}$ is a finitely generated group quasi-isometric to $G$. By Theorem 1.4, $G^{\prime}$ contains an 2-ended almost normal subgroup $H^{\prime}$ \& $G^{\prime}$. 
Since 2-ended groups are virtually cyclic, we may assume by Proposition 3.11 that $H^{\prime}=\langle t\rangle$ for some $t \in G^{\prime}$. By Proposition 5.5 and Lemma 5.8, it follows that the associated height function $h^{\prime}: G^{\prime} \rightarrow \mathbb{R}$ has bounded image. Since $(\mathbb{R},+)$ is torsion-free, Proposition 5.9 ensures $h^{\prime}$ is trivial.

Let $S=\left\{s_{1}, s_{2}, \ldots, s_{n}\right\}$ be a finite generating set of $G^{\prime}$. Since $s_{i} H s_{i}^{-1}$ and $H$ are commensurable, there are positive integers $n_{i}$ and $m_{i}$ such that $s_{i} t^{m_{i}} s_{i}^{-1}=t^{ \pm n_{i}}$. As $h^{\prime}$ is trivial, $n_{i}= \pm m_{i}$. Thus for sufficiently large $M$ such that $m_{i} \mid M$ for all $i$, we deduce that $s_{i}\left\langle t^{M}\right\rangle s_{i}^{-1}=\left\langle t^{M}\right\rangle$ for all $i$. Since $S$ is a generating set, $\left\langle t^{M}\right\rangle$ is an infinite cyclic normal subgroup of $G^{\prime}$.

It is easy to see, as observed in the preceding proof, that central extensions necessarily have bounded fibre distortion. One may wonder if the converse holds: does bounded fibre distortion distortion necessarily imply the group is (virtually) a central extension? This is not the case. The following example resolves a question of [FLS15, Section 12.2], giving an example of a group quasi-isometric to $F_{2} \times \mathbb{Z}^{2}$ that does not have a normal free abelian subgroup of rank 2.

Example 5.11. Let

$$
G=\left\langle a, b, t \mid[a, b], a^{13}=t a^{5} b^{12} t^{-1}, b^{13}=t a^{-12} b^{5} t^{-1}\right\rangle .
$$

This is an HNN extension of $\mathbb{Z}^{2}$ with stable letter $t$. Notice that the associated matrix

$$
A:=\left(\begin{array}{cc}
\frac{5}{13} & -\frac{12}{13} \\
\frac{12}{13} & \frac{5}{13}
\end{array}\right)
$$

is an infinite order rotation through angle $\arccos \left(\frac{5}{13}\right)$. Thus the $\mathbb{Z}^{2}$ subgroup $H=\langle a, b\rangle \leq G$ is almost normal but not normal. We now build a graph of spaces $X$ by taking two tori $T$ and $T^{\prime}$ and gluing $T^{\prime} \times[0,1]$ to $T$ so that $\pi(X)=G$. Endowing $T$ and $T^{\prime} \times[0,1]$ with standard Euclidean metrics, we see that as $A$ is orthogonal, the attaching maps used to define $X$ are isometric embeddings. Thus $\tilde{X}$ is isometric to $\mathbb{E}^{2} \times T$, where $T$ is a regular tree with infinitely many ends, and so $G$ is quasi-isometric to $F_{2} \times \mathbb{Z}^{2}$. Such examples are considered in work of Leary and Minasyan [LM19]. See also HP18.

We now investigate what one can say if the fibre distortion function is unbounded. In particular, we are interested in the following situation:

Definition 5.12. Let $G$ be a finitely generated group containing an almost normal subgroup $H \& G$. We say that $(G, H)$ has proper fibre distortion if the fibre distortion map $F: G / H \rightarrow \mathbb{R}$ is proper, i.e. for each $r \geq 0$, $F^{-1}([1, r])$ is finite.

Example 5.13. Suppose $G$ is a finitely generated group extension

$$
1 \rightarrow H \cong \mathbb{Z}^{n} \rightarrow G \rightarrow Q \rightarrow 1
$$

such that the map $Q \rightarrow A u t(H) \cong G L(n, \mathbb{Z})$ has finite kernel. In such a situation, we say that $G$ is $\mathbb{Z}^{n}$-by-( $\infty$ ended) with almost injective quotient (AIQ). Observe that for every $r \geq 0$, there are only finitely many matrices $M \in G L(n, \mathbb{Z})$ such that $\|M\|_{1} \leq r$. Thus Example 5.4 ensures that $(G, H)$ has proper fibre distortion if and only if $G$ is $\mathbb{Z}^{n}-$ by-( $\infty$ ended) with AIQ. 
Proposition 5.5 implies the following:

Corollary 5.14. Suppose that $G$ and $G^{\prime}$ are groups containing almost normal subgroups $H$ \& $G$ and $H^{\prime}$ \& $G^{\prime}$ such that the hypotheses of Theorem 1.4 are satisfied. If $G$ and $G^{\prime}$ are quasi-isometric, then $(G, H)$ has proper fibre distortion if and only if $\left(G^{\prime}, H^{\prime}\right)$ does.

The following is a generalisation of Lemma 4.1 of Why10:

Proposition 5.15. Suppose $G$ is finitely presented, $\mathbb{Z}^{n} \cong H$ a $G$ and $e(G / H)=$ $\infty$. Suppose $(G, H)$ has proper fibre distortion. Then $H$ has a finite index subgroup that is normal in $G$.

Proof. Let $F: G / H \rightarrow \mathbb{R}$ be the fibre distortion map. It follows from the definition of $A_{g}$ in Example 5.1 that $A_{g k}=A_{k} A_{g}$ for all $g, k \in G$. Suppose $g \in G$ and $h \in H$. Using Example [5.1, we see that

$$
\sup _{k \in H} d\left(k, g h g^{-1}\left\lfloor A_{g^{-1}} A_{h} A_{g} \cdot k\right\rfloor\right)=\sup _{k \in H} d\left(k, g h g^{-1}\left\lfloor A_{g h g^{-1}} \cdot k\right\rfloor\right)<\infty .
$$

Since $h \in H, A_{h}$ is the identity, so $A_{g^{-1}} A_{h} A_{g}$ is also identity. By Example 5.4, we see that $F\left(g h g^{-1} H\right)=0$.

Let $X$ be the 2-complex with vertex set $G / H$ constructed in the proof of Theorem 3.24. As in the proof of Theorem 3.24, there exists a collection of tracks $\left\{\tau_{i}\right\}$ in $X$ such that components of $X \backslash \cup_{i} \tau_{i}$ correspond to vertices of the dual tree $T$. We claim that $H$ fixes $T$. If this is the case, then let $K$ be the kernel of the action of $G$ on $T$. By assumption $H \leq K$. Since edge stabilisers are commensurable to $H$ and $K$ is contained in an edge stabiliser, it follows that $K$ is commensurable to $H$. Since $K$ is normal, $H$ has a finite index normal subgroup.

To prove the claim, we suppose some $h \in H$ doesn't fix $T$. Let $v$ be the vertex of $T$ corresponding to the component of $X \backslash \cup_{i} \tau_{i}$ containing $H$. Then $H v=v$. Thus there is an $A \geq 0$ and a component $C$ of $T \backslash N_{A}(v)$ such that $h C \neq C$. Since $G \backslash T$ is cocompact, we can pick a sequence $\left(g_{i}\right)$ in $G$ such that $g_{i} v \in C$ for all $i$ and $\lim _{i \rightarrow \infty} d_{T}\left(v, g_{i} v\right)=\infty$. As any path from $g_{i} v$ to $h g_{i} v$ passes through $N_{A}(v)$, we see that

$$
d_{T}\left(v, g_{i}^{-1} h g_{i} v\right)=d_{T}\left(g_{i} v, h g_{i} v\right) \geq d_{T}\left(g_{i} v, v\right)+d_{T}\left(g_{i} v, v\right)-2 A .
$$

Thus $\lim _{i \rightarrow \infty} d_{T}\left(v, g_{i}^{-1} h g_{i} v\right)=\infty$. By passing to a subsequence, we may assume that $g_{i}^{-1} h g_{i} v \neq g_{j}^{-1} h g_{j} v$ unless $i=j$. Since $H v=v$, we deduce that $g_{i}^{-1} h g_{i} H \neq g_{j}^{-1} h g_{j} H$ when $i \neq j$. By the first paragraph, we have $F\left(g_{i}^{-1} h g_{i} H\right)=0$ for all $i$, which contradicts the properness of $F$.

We now combine Theorem 1.4 with Propositions 5.5 and 5.15 to deduce quasi-isometric rigidity for the class of $\mathbb{Z}^{n}$-by-( $\infty$ ended) groups with AIQ:

Theorem 5.16. Let $G$ be a group of type $F_{n+1}$ that is $\mathbb{Z}^{n}-$ by-( $\infty$ ended) with $A I Q$. If $G^{\prime}$ is any finitely generated group quasi-isometric to $G$, then it is also $\mathbb{Z}^{n}-$ by-( $\infty$ ended) with $A I Q$. Moreover, any quasi-isometry $f$ : $G \rightarrow G^{\prime}$ induces a quasi-isometry $G / \mathbb{Z}^{n} \rightarrow G^{\prime} / \mathbb{Z}^{n}$ between the infinite ended quotients. 


\section{Appendix A. QI RIGIDITY of SURFACE GROUP EXTEnsions}

Throughout this appendix, let $S$ denote a closed hyperbolic surface. In FM02a, Farb and Mosher prove quasi-isometric rigidity for hyperbolic surface-by-free groups. These are group extensions of form $1 \rightarrow \pi_{1}(S) \rightarrow$ $\Gamma_{H} \rightarrow K \rightarrow 1$, where the induced action $K \leq \operatorname{Out}\left(\pi_{1}(S)\right) \cong \operatorname{MCG}(S)$ is a Schottky subgroup, i.e. a convex cocompact free subgroup in the sense of [FM02b]. These methods were further developed by Mosher Mos03a, Mos09 to study the coarse geometry of other surface group extensions.

Mosher classified fibre-preserving quasi-isometries of certain surface group extensions in [Mos03a]. Theorem 4.1 tells us that in many cases, every quasiisometry is fibre-preserving. Combining these results allows us to deduce Theorem A.19, For the convenience of the reader, we give an account of Mosher's methods and explain how to deduce Theorem A.19 from Theorem 4.1 and Mos03a]. We refer the reader to [FM02b], FM02a and Mos03a] for more details on these topics.

Background on Teichmüller space. Teichmüller space is defined to be the space of marked hyperbolic structures on $S$ modulo isotopy. Equivalently, it is the space of marked conformal structures on $S$ modulo isotopy. A measured foliation on $S$ is defined to be a foliation $\mathcal{F}$ with finitely many singular points such that:

(1) each neighbourhood of a singular point is a $k$-pronged saddle for $k \geq 3$ (see [FM12]);

(2) $\mathcal{F}$ is equipped with a positive transverse Borel measure.

A saddle collapse of a measured foliation $\mathcal{F}$ is obtained by collapsing the leaf segment joining two singularities to a point. The set of measured foliations of $S$, modulo isotopy and saddle collapse, is denoted $\mathcal{M F}$. Given a measured foliation $\mathcal{F}$ and $\lambda>0$, let $\lambda \mathcal{F}$ be the measured foliation obtained by multiplying the transverse measure by $\lambda$. Let $\mathbf{P} \mathcal{M F}$ denote the set of all projective measured foliations, i.e. $\mathcal{M F}$ modulo the equivalence relation $\lambda F \sim F$ for $\lambda>0$. We let $[\mathcal{F}] \in \mathbf{P} \mathcal{M F}$ denote the equivalence class containing $\mathcal{F}$.

We define a topology on $\mathbf{P} \mathcal{M F}$ and $\mathcal{T}$ as follows. Let $\mathcal{C}$ denote the set of isotopy classes of simple closed curves on $S$ and let $\mathbf{P}[0, \infty)^{\mathcal{C}}$ be the projectivisation of $[0, \infty)^{\mathcal{C}}$. There is an injection $\mathcal{T} \rightarrow \mathbf{P}[0, \infty)^{\mathcal{C}}$ that associates to each $x \in \mathcal{T}$ and $c \in \mathcal{C}$ the length of the unique geodesic in the class $c$. The image of this map is homeomorphic to an open ball of dimension $6 g-6$. For each measured foliation $\mathcal{F}$ and $c \in \mathcal{C}$, we define $\ell_{\mathcal{F}}(c)$ to be the infimum of $\int_{\gamma} \mathcal{F}$ as $\gamma$ ranges over curves in the isotopy class $c$. The map $\mathcal{F} \mapsto\left(c \mapsto \ell_{\mathcal{F}}(c)\right)$ descends to a well-defined map $\mathbf{P} \mathcal{M} \mathcal{F} \rightarrow \mathbf{P}[0, \infty)^{\mathcal{C}}$ with image homeomorphic to a sphere of dimension $6 g-7$. We topologise $\mathcal{T}$ and $\mathbf{P} \mathcal{M F}$ by identifying them with their images in $\mathbf{P}[0, \infty)^{\mathcal{C}}$ endowed with subspace topology. Then Thurston's compactification theorem says that with respect to the above embedding into $\mathbf{P}[0, \infty)^{\mathcal{C}}, \overline{\mathcal{T}}=\mathcal{T} \sqcup \mathbf{P} \mathcal{M F}$ is the closure of $\mathcal{T}$.

We now describe the Teichmüller metric on $\mathcal{T}$. We say that two measured foliations $\mathcal{F}_{x}$ and $\mathcal{F}_{y}$ are transverse if:

(1) they have the same set of singular point; 
(2) each singular point has the same number of prongs;

(3) their leaves are transverse at all regular points.

Two transverse measured foliations $\mathcal{F}_{x}$ and $\mathcal{F}_{y}$ determine a conformal structure at regular points of $S$ : a neighbourhood of each regular point is identified with a subset of $\mathbb{C}$ such that leaves of $\mathcal{F}_{x}$ (resp. $\mathcal{F}_{y}$ ) correspond to lines with constant imaginary (resp. real) part and distance is determined by the transverse measures. This conformal structure extends uniquely over singular points to a conformal structure on $S$ itself, hence determines a point of $\mathcal{T}$ which we denote $\sigma\left(\mathcal{F}_{x}, \mathcal{F}_{y}\right)$. This is well-defined for any pair of transverse measured foliations $\left(\mathcal{F}_{x}, \mathcal{F}_{y}\right)$ that represent the same pair in $\mathbf{P} \mathcal{M} \mathcal{F}^{2}$.

A pair of transverse measured foliations $\left(\mathcal{F}_{x}, \mathcal{F}_{y}\right)$ defines a Teichmüller geodesic

$$
t \mapsto \sigma\left(e^{t} \mathcal{F}_{x}, e^{-t} \mathcal{F}_{y}\right),
$$

which we denote $\overleftrightarrow{\left.\left[\mathcal{F}_{x}\right],\left[\mathcal{F}_{y}\right]\right)}$. Teichmüller's theorem says that every pair of points on Teichmüller space lie on a unique Teichmüller geodesic, and so the associated Teichmüller metric given by $d\left(\sigma\left(e^{t} \mathcal{F}_{x}, e^{-t} \mathcal{F}_{y}\right), \sigma\left(e^{s} \mathcal{F}_{x}, e^{-s} \mathcal{F}_{y}\right)\right)=$ $|s-t|$ is a well-defined metric on $\mathcal{T}$. The topology induced by this metric agrees with the topology on $\mathcal{T}$ defined by homeomorphically identifying $\mathcal{T}$ with a subset of $\mathbf{P}[0, \infty)^{\mathcal{C}}$ via the preceding embedding. Henceforth, it will be assumed that $\mathcal{T}$ is endowed with the Teichmüller metric.

The mapping class group and its action on Teichmüller space. The mapping class group $\mathrm{MCG}(S)$ is the group $\operatorname{Homeo}(S) / \operatorname{Homeo}_{0}(S)$, where Homeo $(S)$ is the group of homeomorphisms (not necessarily orientation preserving) of $S$ and $\mathrm{Homeo}_{0}(S)$ is the connected component of the identity. This is sometimes called the extended mapping class group of $S$. The mapping class group $\operatorname{MCG}(S)$ acts properly discontinuously and isometrically on $\mathcal{T}(S)$, but the action is not cocompact.

Let $p \in S$ be a point. The Dehn-Nielsen-Baer says that there is an isomorphism of short exact sequences, where the top row is known as the Birman exact sequence associated to the mapping class group.

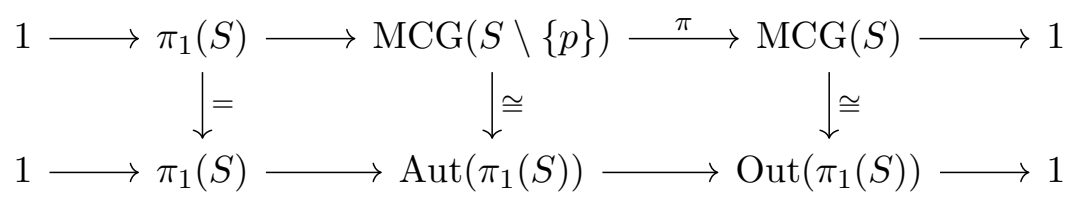

A homeomorphism $H \in \operatorname{Homeo}(S)$ is said to be a pseudo-Anosov if there exist a pair of transverse measured foliations $\left(\mathcal{F}_{x}, \mathcal{F}_{y}\right)$ and a $\lambda>1$ such that $f\left(\mathcal{F}_{x}, \mathcal{F}_{y}\right)=\left(\lambda \mathcal{F}_{x}, \lambda^{-1} \mathcal{F}_{y}\right)$. A mapping class $f \in \operatorname{MCG}(S)$ is said to be pseudo-Anosov if it has a pseudo-Anosov representative. Every pseudoAnosov $g$ acts by translations of non-zero length along a Teichmüller geodesic. This geodesic is unique and is called the axis of $g$.

This action extends to an action $\operatorname{MCG}(S) \curvearrowright \overline{\mathcal{T}(S)}$. Given $f \in \operatorname{MCG}(S)$, let $\operatorname{Fix}(f)$ denote the set of fixed points of $f$ in $\overline{\mathcal{T}(S)}$. If $f$ is a pseudoAnosov such that $f\left(\mathcal{F}_{x}, \mathcal{F}_{y}\right)=\left(\lambda \mathcal{F}_{x}, \lambda^{-1} \mathcal{F}_{y}\right)$ for some $\lambda>1$, then $\operatorname{Fix}(f)=$ $\left\{\left[\mathcal{F}_{x}\right],\left[\mathcal{F}_{y}\right]\right\}$. 
Lemma A.1 ([McC94]). Given two pseudo-Anosovs $f, g \in \operatorname{MCG}(S)$, either $\operatorname{Fix}(f)=\operatorname{Fix}(g)$ or $\operatorname{Fix}(f) \cap \operatorname{Fix}(g)=\emptyset$. The setwise stabiliser of $\operatorname{Fix}(f)$ is equal to $\operatorname{Comm}(\langle f\rangle)$ and contains $\langle f\rangle \cong \mathbb{Z}$ as a finite index subgroup.

We say that $L \leq \operatorname{MCG}(S)$ is irreducible if it doesn't preserve a finite collection of disjoint simple closed curves. Ivanov showed that a subgroup of $\operatorname{MCG}(S)$ is infinite and irreducible if and only if it contains a pseudoAnosov [Iva92, Corollary 7.14].

Given a measured foliation $\mathcal{F}$ of $S$, we can lift it to a $\pi_{1}(S)$-invariant measured foliation $\widetilde{\mathcal{F}}$ of $\widetilde{S} \cong \mathbb{H}^{2}$. We set $E([\mathcal{F}]) \subseteq S^{1}$ to be the set of endpoints of leaves of $\widetilde{\mathcal{F}}$, noting that $E([\mathcal{F}])$ is independent of the choice of $\mathcal{F} \in[\mathcal{F}]$. If $\left(\mathcal{F}_{x}, \mathcal{F}_{y}\right)$ is a pair of transverse measured foliations, then $E\left(\left[\mathcal{F}_{x}\right]\right) \cap E\left(\left[\mathcal{F}_{y}\right]\right)=\emptyset$ by transversality of $\mathcal{F}_{x}$ and $\mathcal{F}_{y}$. Thus if $\left[\mathcal{F}_{x}\right],\left[\mathcal{F}_{y}\right] \in$ $\mathbf{P} \mathcal{M} \mathcal{F}$ can be joined by a Teichmüller geodesic, then $E\left(\left[\mathcal{F}_{x}\right]\right) \cap E\left(\left[\mathcal{F}_{y}\right]\right)=\emptyset$.

Let $g$ be a pseudo-Anosov with $\operatorname{Fix}(g)=\left\{\left[\mathcal{F}_{x}\right],\left[\mathcal{F}_{y}\right]\right\}$. We define $E(g):=$ $E\left(\left[\mathcal{F}_{x}\right]\right) \sqcup E\left(\left[\mathcal{F}_{y}\right]\right)$. Suppose that $f$ is another pseudo-Anosov such that $\operatorname{Fix}(f) \cap \operatorname{Fix}(g)=\emptyset$. Then any two distinct elements of $\operatorname{Fix}(f) \cup \operatorname{Fix}(g)$ can be joined by a Teichmüller geodesic. To see this, we recall from [FM02b] that for $n$ sufficiently large, the subgroup generated by $\left\langle f^{n}, g^{n}\right\rangle$ is convex cocompact. The definition of convex cocompactness then ensures that any two elements of $\operatorname{Fix}\left(f^{n}\right) \cup \operatorname{Fix}\left(g^{n}\right)=\operatorname{Fix}(f) \cup \operatorname{Fix}(g)$ can be joined by a Teichmüller geodesic. Hence we deduce the following:

Lemma A.2. If $f, g \in \mathrm{MCG}(S)$ are pseudo-Anosovs, then either $E(f) \cap$ $E(g)=\emptyset$ or $E(f)=E(g)$. In the latter case, $g \in \operatorname{Comm}(\langle f\rangle)$ and $f$ and $g$ share an axis.

A subset $A \subseteq \mathcal{T}$ is said to be cobounded if there is some bounded set $\Omega \subseteq \mathcal{T}$ such that $A \subseteq \operatorname{MCG}(S) \Omega$. Every axis of a pseudo-Anosov is cobounded. We deduce the following lemma via Proposition 3.3 of [AAS07], which also follows from the proof of [FM02b, Lemma 2.4].

Lemma A.3. Let $\gamma=\overleftrightarrow{(u, v)}$ and $\gamma^{\prime}=\overleftrightarrow{\left(u^{\prime}, v^{\prime}\right)}$ be cobounded Teichmüller geodesics such that $\{u, v\} \cap\left\{u^{\prime}, v^{\prime}\right\}=\emptyset$. Then for any $r \geq 0$, the set $\{\sigma \in$ $\left.\mathcal{T} \mid d(\sigma, \gamma), d\left(\sigma, \gamma^{\prime}\right) \leq r\right\}$ is bounded. In particular, $\gamma$ and $\gamma^{\prime}$ cannot be at finite Hausdorff distance.

We define the canonical marked hyperbolic surface bundle $\mathcal{S} \rightarrow \mathcal{T}$ as follows. The fibre $S_{\sigma}$ is the surface $S$ equipped with a hyperbolic metric corresponding to $\sigma \in \mathcal{T}$, chosen so that $S_{\sigma}$ varies smoothly with $\sigma$. The canonical hyperbolic plane bundle $p: \mathcal{H} \rightarrow \mathcal{T}$ is the universal cover of $\mathcal{S}$, where each fibre is a hyperbolic plane identified with the universal cover of each $S_{\sigma}$. In fact, $\mathcal{H}$ can be identified with the Teichmüller space of the punctured surface $S \backslash p$, and the mapping class group of $S \backslash p$ admits a fibre-preserving isometric action on $\mathcal{H}$. Let $D_{\sigma}=p^{-1}(\sigma)$ denote the fibre at $\sigma \in \mathcal{T}$.

A line $\ell \subseteq \mathcal{T}$ is the image of a piecewise geodesic map $\mathbb{R} \rightarrow \mathcal{T}$. We can pull back the canonical hyperbolic plane bundle to get a bundle $\mathcal{H}_{\ell} \rightarrow \ell$. Each $\mathcal{H}_{\ell}$ can be naturally endowed with a piecewise Riemannian metric, see for instance [FM02b]. A line is said to be hyperbolic if $\mathcal{H}_{\ell}$ is (Gromov) 
hyperbolic. We make use of the following theorem, proven independently by Bowditch and Mosher:

Theorem A.4 (Mos03b, Bow13]). Let $Z \subseteq \mathcal{T}$ be cobounded and let $\delta \geq 0$. There is a constant $N$ such that the following holds. If $\ell \subseteq Z$ is a line such that $\mathcal{H}_{\ell}$ is $\delta$-hyperbolic, then there is a cobounded Teichmüller geodesic $\gamma_{\ell}$ such that $d_{\text {Haus }}\left(\gamma_{\ell}, \ell\right) \leq N$. Conversely, if a line $\ell \subseteq Z$ is Hausdorff equivalent to a cobounded geodesic $\gamma$, then the bundle $\mathcal{H}_{\ell}$ is hyperbolic.

Given a cobounded geodesic $\gamma \subseteq \mathcal{T}$, the bundle $\mathcal{H}_{\gamma} \rightarrow \gamma$ also admits a singular Solv metric, which we denote $\mathcal{H}_{\gamma}^{\text {Solv }}$. If $\left(\mathcal{F}_{x}, \mathcal{F}_{y}\right)$ is a pair of transverse measured foliations such that $\gamma=\overleftrightarrow{\left(\left[\mathcal{F}_{x}\right],\left[\mathcal{F}_{y}\right]\right)}$ and $\gamma$ is parametrised by $t$, this SoLv metric is defined by $d s^{2}=e^{-2 t} d x^{2}+e^{2 t} d y^{2}+d t^{2}$, where $d x$ and $d y$ are the transverse measures associated to $\widetilde{\mathcal{F}_{x}}$ and $\widetilde{\mathcal{F}}_{y}$. It is shown in Proposition 4.4 of FM02a that the identity map $\mathcal{H}_{\gamma} \rightarrow \mathcal{H}_{\gamma}^{\text {Solv }}$ is a quasiisometry.

Let $\operatorname{Isom}\left(\mathcal{H}_{\gamma}^{\text {Solv }}\right)$ denote the group of isometries of $\mathcal{H}_{\gamma}^{\text {Solv }}$. It is shown in FM02a that isometries of $\mathcal{H}_{\gamma}^{\mathrm{SoLV}}$ are fibre-preserving, i.e. if $\phi: \mathcal{H}_{\gamma}^{\mathrm{Solv}} \rightarrow$ $\mathcal{H}_{\gamma}^{\text {Solv }}$ is an isometry then $\phi\left(D_{\sigma}\right)=D_{\bar{\phi}(\sigma)}$ for every $\sigma \in \gamma$, where $\bar{\phi}: \gamma \rightarrow \gamma$ is an induced isometry of $\gamma$. Thus there is a short exact sequence

$$
1 \rightarrow \operatorname{Isom}_{h}\left(\mathcal{H}_{\gamma}^{\text {SoLv }}\right) \rightarrow \operatorname{Isom}\left(\mathcal{H}_{\gamma}^{\text {SoLv }}\right) \stackrel{\phi \mapsto \bar{\phi}}{\longrightarrow} C_{\gamma} \rightarrow 1
$$

where $\operatorname{Isom}_{h}\left(\mathcal{H}_{\gamma}^{\text {SOLV }}\right)$ denotes the kernel of the induced action on $\gamma$ and $C_{\gamma}$ is the associated quotient. It follows from FM02a that $C_{\gamma}$ is isomorphic to either $1, \mathbb{Z}_{2}, \mathbb{Z}$ or $D_{\infty}$ and the restriction of $\operatorname{Isom}_{h}\left(\mathcal{H}_{\gamma}^{\text {Solv }}\right)$ to any fibre $D_{\sigma}$ is a cocompact group of isometries of $D_{\sigma} \cong \mathbb{H}^{2}$ that contains $\pi_{1}(S)$ as a finite index subgroup.

If $\gamma$ and $\gamma^{\prime}$ are two Teichmüller geodesics, a quasi-isometry $f: \mathcal{H}_{\gamma} \rightarrow \mathcal{H}_{\gamma^{\prime}}$ is said to be fibre-preserving if there is a constant $B$ such that for each $\sigma \in \gamma$, there is some $\mu \in \gamma^{\prime}$ with $d_{\text {Haus }}\left(f\left(D_{\sigma}\right), D_{\mu}\right) \leq B$.

Proposition A.5 ([FM02a, Lemma 6.3]). Let $\gamma$ and $\gamma^{\prime}$ be cobounded Teichmüller geodesics in $\mathcal{T}$ such that $\gamma$ is the axis of a pseudo-Anosov. If there exists a fibre-preserving quasi-isometry $f: \mathcal{H}_{\gamma} \rightarrow \mathcal{H}_{\gamma^{\prime}}$, then $\gamma^{\prime}$ is also the axis of a pseudo-Anosov and there exists an isometry $\mathcal{H}_{\gamma}^{\text {SoLv }} \rightarrow \mathcal{H}_{\gamma^{\prime}}^{\text {Solv }}$.

Much of the theory of mapping class group and Teichmüller space of surfaces extends to the setting of orbifolds, as observed in [FM02b] and FM02a. Throughout, we will restrict our attention to closed hyperbolic 2orbifolds with only cone singularities, since these are the only orbifolds that admit pseudo-Anosov homeomorphisms. Let $\mathcal{O}$ be such an orbifold and let $\pi_{1}(\mathcal{O})$ denote the orbifold fundamental group. As shown in FM02b, an analogue of the Dehn-Nielsen-Baer theorem holds so that $\operatorname{Out}\left(\pi_{1}(\mathcal{O})\right) \cong$ $\operatorname{MCG}(\mathcal{O})$. However, it is not the case that $\operatorname{Aut}\left(\pi_{1}(\mathcal{O})\right) \cong \operatorname{MCG}(\mathcal{O} \backslash\{p\})$. We define $\widetilde{\mathrm{MCG}}(\mathcal{O})$ to be the group of all lifts of homeomorphisms of $\mathcal{O}$ to the universal cover $\widetilde{\mathcal{O}}$, modulo the connected component of the identity. In [FM02b], it was shown that there is an isomorphism of short exact sequences 
as follows:

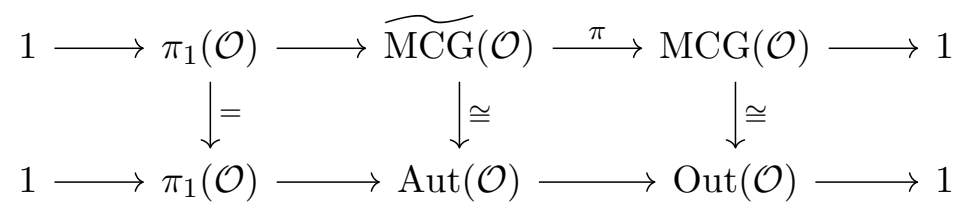

Quasi-symmetric homeomorphisms of the circle.

Definition A.6. Let $M \geq 1$. A homeomorphism $h: S^{1} \rightarrow S^{1}$ is said to be $M$-quasi-symmetric if for all $x \in \mathbb{R}$ and $t \in(0,2 \pi)$, we have

$$
\frac{1}{M} \leq\left|\frac{h\left(e^{i(x+t)}\right)-h\left(e^{i x}\right)}{h\left(e^{i x}\right)-h\left(e^{i(x-t)}\right)}\right| \leq M .
$$

We say that a homeomorphism $h: S^{1} \rightarrow S^{1}$ is quasi-symmetric if it is $M$-quasi-symmetric for some $M \geq 1$.

The set of quasi-symmetric homeomorphisms of $S^{1}$ is closed under composition and taking inverses. Hence quasi-symmetric homeomorphisms of $S^{1}$ form a group which we denote $\operatorname{QSym}\left(S^{1}\right)$. The following theorem is essentially due to Ahlfors-Beurling [BA56], see also [Pau96].

Theorem A.7 ([BA56, Pau96]). There is an isomorphism $\Lambda: \mathrm{QIsom}\left(\mathbb{H}^{2}\right) \cong$ $\operatorname{QSym}\left(S^{1}\right)$ such that the following hold:

(1) For $K \geq 1$ and $A \geq 0$, there is an $M=M(K, A)$ such that every $(K, A)$-quasi-isometry $f: \mathbb{H}^{2} \rightarrow \mathbb{H}^{2}$ induces an $M$-quasi-symmetric homeomorphism $\Lambda(f): S^{1} \rightarrow S^{1}$.

(2) If $f, g \in \mathrm{QIsom}\left(\mathbb{H}^{2}\right)$ such that $\sup _{x \in \mathbb{H}^{2}} d(f(x), g(x))<\infty$, then $\Lambda(f)=\Lambda(g)$.

(3) If $M \geq 1$, there are $K=K(M)$ and $A=A(M)$ such that every $M$-quasi-symmetric homeomorphism $\lambda: S^{1} \rightarrow S^{1}$ induces a $(K, A)$ quasi-isometry $f: \mathbb{H}^{2} \rightarrow \mathbb{H}^{2}$ such that $\Lambda(f)=\lambda$.

(4) If $f, g \in \mathrm{QIsom}\left(\mathbb{H}^{2}\right)$ are $(K, A)$-quasi-isometries such that $\Lambda(f)=$ $\Lambda(g)$, then $\sup _{x \in \mathbb{H}^{2}} d(f(x), g(x)) \leq B$ for some $B=B(K, A)$.

We fix some cocompact lattice $\Gamma \leq \operatorname{Isom}\left(\mathbb{H}^{2}\right)$. Note that $\Gamma$ is a hyperbolic group with boundary $S^{1}$. Thus every automorphism $\Gamma \rightarrow \Gamma$ canonically induces a quasi-symmetric homeomorphism $S^{1} \rightarrow S^{1}$. This gives a homomorphism $\theta: \operatorname{Aut} \Gamma \rightarrow \operatorname{QSym}\left(S^{1}\right)$. The following lemma is implicit in Nielsen's work on surface group automorphisms.

Lemma A.8. The map $\theta:$ Aut $\Gamma \rightarrow \operatorname{QSym}\left(S^{1}\right)$ is injective.

Proof. Let $\alpha \in$ Aut $\Gamma$ be an automorphism such that the induced map $\theta(\alpha)$ is trivial. Fix some $g \in \Gamma$. For each $h \in \Gamma$, let $C_{h}: \Gamma \rightarrow \Gamma$ be the inner automorphism $k \mapsto h k h^{-1}$. Applying $\theta$ to the identity

$$
C_{\alpha(g)} \circ \alpha \circ C_{g^{-1}} \circ \alpha^{-1}=\operatorname{id}_{G},
$$

we deduce that $\theta\left(C_{\alpha(g) g^{-1}}\right)=\operatorname{id}_{S^{1}}$ for all $g \in \Gamma$. Hence $\alpha(g) g^{-1}$ is an isometry of $\mathbb{H}^{2}$ which extends to a map that fixes the boundary. However any isometry of $\mathbb{H}^{2}$ that induces the identity map on the boundary must be trivial. Therefore $\alpha(g)=g$ for all $g \in \Gamma$. 
We thus identify Aut $\Gamma$ with a subgroup of $\operatorname{QSym}\left(S^{1}\right)$, which we also denote Aut $\Gamma$. Moreover, as $\Gamma$ has trivial centre, it may also be identified with a subgroup of Aut $\Gamma \leq \operatorname{QSym}\left(S^{1}\right)$.

Lemma A.9. If there exists an $f \in \mathrm{QSym}\left(S^{1}\right)$ such that $f \circ g \circ f^{-1}=g$ for every $g \in \Gamma \leq \operatorname{QSym}\left(S^{1}\right)$, then $f=\operatorname{id}_{S^{1}}$.

Proof. Let $g \in \Gamma$ and $x \in \operatorname{Fix}(g) \subseteq S^{1}$. As $f(x)=f(g(x))=(f \circ g \circ$ $\left.f^{-1}\right)(f(x))=g(f(x))$, we deduce that $f(x) \in \operatorname{Fix}(g)$.

Let

$$
\Lambda:=\left\{x \in S^{1} \mid x \in \operatorname{Fix}(g) \text { for some loxodromic } g \in \Gamma\right\} .
$$

Since $\Lambda$ is a dense subset of $S^{1}$, it is sufficient to show that $\left.f\right|_{\Lambda}=\operatorname{id}_{\Lambda}$. For any $x \in \Lambda$, let $g, h \in \Gamma$ be loxodromic elements with $x \in \operatorname{Fix}(g)$ and $\operatorname{Fix}(g) \cap$ $\operatorname{Fix}(h)=\emptyset$. Suppose $\operatorname{Fix}(g)=\{x, y\}$ and $\operatorname{Fix}(h)=\{w, z\}$. Replacing $g$ with $g^{-1}$ if necessary, we may suppose that $x$ is the attracting fixed point of $g$. We note that $\operatorname{Fix}\left(g^{n} h g^{-n}\right)=\left\{g^{n} w, g^{n} z\right\}$. Since $x$ is the attracting fixed point of $g$ and $w, z \neq y$, it follows that $g^{n} w \rightarrow x$ and $g^{n} z \rightarrow x$ as $n \rightarrow \infty$. By the above observation that $f\left(g^{n} w\right)=g^{n} w$ or $g^{n} z$, we see that $f\left(g^{n} w\right) \rightarrow x$ as $n \rightarrow \infty$, thus $f(x)=x$ by continuity of $f$.

Corollary A.10. The inclusion

$$
\theta: \operatorname{Aut} \Gamma \rightarrow \operatorname{Norm}_{\mathrm{QSym}\left(S^{1}\right)}(\Gamma):=\left\{f \in \operatorname{QSym}\left(S^{1}\right) \mid f \Gamma f^{-1}=\Gamma\right\}
$$

is surjective.

Proof. Suppose there exists an $f \in \operatorname{QSym}\left(S^{1}\right)$ with $f \Gamma f^{-1}=\Gamma$. There is an automorphism $\alpha \in$ Aut $\Gamma$ such that for all $g \in \Gamma, f \theta(g) f^{-1}=\theta(\alpha(g))$. By identity (44) in Lemma A.8, we see that $\theta(\alpha(g))=\theta(\alpha) \theta(g) \theta\left(\alpha^{-1}\right)$. Hence $\theta(\alpha)^{-1} f \theta(g) f^{-1} \theta(\alpha)=\theta(g)$ for all $g \in \Gamma$. Thus Lemma A.9 ensures that $\theta(\alpha)=f$.

Proof of quasi-isometric rigidity of surface group extensions. We fix a closed hyperbolic surface $S$ and a finitely generated, infinite ended, irreducible subgroup $L \leq \operatorname{MCG}(S)$ of type $F_{3}$. Let $\Gamma_{L}$ be the group extension

$$
1 \rightarrow \pi_{1}(S) \rightarrow \Gamma_{L} \stackrel{\pi}{\rightarrow} L \rightarrow 1 \text {. }
$$

By Brown's criterion Bro87, $\Gamma_{L}$ is also of type $F_{3}$. (See also Exercise 1 of Section 7.2 in Geo08.) We now construct a geometric model of the group $\Gamma_{L}$, the details of which are found in [FM02b].

We first fix a generating set of $L$ and embed the correspond Cayley graph $X_{L}$ into $\mathcal{T}$ as follows. We identify the vertex set of $X_{L}$ with some $L$-orbit in $\mathcal{T}$. For each edge in $X_{L}$, we join the corresponding points of $\mathcal{T}$ by a piecewise geodesic, all of which are disjoint except at their endpoints. This is done in an equivariant way. The action of $L$ on $X_{L}$ is cocompact and properly discontinuous. Moreover, the inclusion $X_{L} \rightarrow \mathcal{T}$ is a coarse embedding. For ease of notation, we identify $X_{L}$ with its image in $\mathcal{T}$. We now pullback the canonical hyperbolic plane bundle to obtain the bundle $p_{L}: \mathcal{H}_{L} \rightarrow X_{L}$. As in [FM02b], we endow $\mathcal{H}_{L}$ with a piecewise Riemannian metric such that $\Gamma_{L}$ acts properly discontinuously and cocompactly on $\mathcal{H}_{L}$.

Given a pseudo-Anosov $g \in L$, we say that a line $\ell_{g} \subseteq \mathcal{T}$ is a coarse axis of $g$ if there is a bounded set $\Omega \subseteq \mathcal{T}$ such that $\ell_{g} \subseteq\langle g\rangle \Omega$. Coarse axes of 
pseudo-Anosovs always exist and are coarsely well-defined, i.e. if $\ell_{g}$ and $\ell_{g}^{\prime}$ are coarse axes of the same pseudo-Anosov $g$, then $d_{\text {Haus }}\left(\ell_{g}, \ell_{g}^{\prime}\right)<\infty$.

Proposition A.11. Let $\gamma \subseteq \mathcal{T}$ be the axis of a pseudo-Anosov $g \in \operatorname{MCG}(S)$ such that $\gamma \subseteq N_{B}\left(X_{L}\right)$ for some $B \geq 0$. Then $g^{n} \in L$ for some $n$ sufficiently large.

Proof. For any $x \in X_{L}, d_{\text {Haus }}(\langle g\rangle \cdot x, \gamma)<\infty$ so that $\langle g\rangle \cdot x \subseteq N_{C}(L \cdot x)$ for some $C \geq 0$. Since the map $\operatorname{MCG}(S) \rightarrow \mathcal{T}$ given by $f \mapsto f \cdot x$ is a coarse embedding, we see that there is some $B^{\prime}$ such that $\langle g\rangle \subseteq N_{B^{\prime}}(L)$ in $\operatorname{MCG}(S)$. Thus $g^{n} \in L$ for some $n$ sufficiently large by [MSW11, Corollary 2.14].

Our first step in proving Theorem A.19 is showing that quasi-isometries of $\mathcal{H}_{L}$ are fibre-preserving. This follows from Theorem 4.1 and Lemma 3.6 . This is the only point where we use the fact that $L$ is infinite ended and of type $F_{3}$.

Proposition A.12. For every $K \geq 1$ and $A \geq 0$, there exist constants $B \geq$ $0, K^{\prime} \geq 1$ and $A^{\prime} \geq 0$ such that the following holds. For every $(K, A)$-quasiisometry $f: \mathcal{H}_{L} \rightarrow \mathcal{H}_{L}$, there exists a $\left(K^{\prime}, A^{\prime}\right)$-quasi-isometry $\hat{f}: X_{L} \rightarrow X_{L}$ such that for every $\sigma \in X_{L}, d_{\text {Haus }}\left(f\left(D_{\sigma}\right), D_{\hat{f}(\sigma)}\right) \leq B$.

Each quasi-isometry $f: \mathcal{H}_{L} \rightarrow \mathcal{H}_{L}$ induces a map $\theta(f) \in \operatorname{QSym}\left(S^{1}\right)$ as follows. We fix a fibre $D_{0} \subseteq \mathcal{H}_{L}$. By Proposition A.12, $f\left(D_{0}\right)$ has finite Hausdorff distance from some fibre $D_{x} \subseteq \mathcal{H}_{L}$. However, since $D_{x}$ has finite Hausdorff distance from $D_{0}$, there is a quasi-isometry $f_{0}: D_{0} \rightarrow D_{0}$ such that $\sup _{y \in D_{0}} d\left(f_{0}(y), f(y)\right)<\infty$. This map is coarsely well-defined, so induces a well-defined map $\theta(f) \in \operatorname{QSym}\left(S^{1}\right)$, where $S^{1}$ is identified with the Gromov boundary of $D_{0}$. This map is independent of the choice of fibre $D_{0}$, so $\theta: \operatorname{QI}\left(\mathcal{H}_{L}\right) \rightarrow \operatorname{QSym}\left(S^{1}\right)$ is a well-defined homomorphism.

Proposition A.13. For any $K \geq 1$ and $A \geq 0$, there exists a $B \geq 0$ such that whenever $f: \mathcal{H}_{L} \rightarrow \mathcal{H}_{L}$ is a $(K, A)$-quasi-isometry with $\theta(f)=$ $\operatorname{id}_{S^{1}}, \sup _{x \in \mathcal{H}_{L}} d(x, f(x)) \leq B$. In particular, $\theta: \operatorname{QI}\left(\mathcal{H}_{L}\right) \rightarrow \operatorname{QSym}\left(S^{1}\right)$ is injective.

To prove this, we make use of another result from [FM02a.

Proposition A.14. Let $g, g^{\prime} \in L$ be two pseudo-Anosovs with coarse axes $\ell_{g}, \ell_{g^{\prime}} \subseteq X_{L}$. Suppose that $f: \mathcal{H}_{L} \rightarrow \mathcal{H}_{L}$ is a quasi-isometry such that $d_{\text {Haus }}\left(\hat{f}\left(\ell_{g}\right), \ell_{g^{\prime}}\right)<\infty$, with $\hat{f}$ as in Proposition A.12. Then $\theta(f)(E(g))=$ $E\left(g^{\prime}\right)$.

Proof. Let $\gamma$ be the axis of $g$ and let $\mathcal{F}_{x}$ and $\mathcal{F}_{y}$ be the associated transverse measured foliations. Then the bundle $\mathcal{H}_{\gamma}^{\text {Solv }}$ has stable and unstable foliations as defined in Section 5 of [FM02a]. These are defined so that the intersection of the stable (resp. unstable) foliation with each fibre $D_{\sigma}$ is $\widetilde{\mathcal{F}}_{y}$ (resp. $\widetilde{\mathcal{F}_{x}}$ ). If $\gamma^{\prime}$ is the axis of $g^{\prime}$, then $\mathcal{H}_{\gamma^{\prime}}^{\text {Solv }}$ also has stable and unstable foliations.

Since $d_{\text {Haus }}\left(\hat{f}\left(\ell_{g}\right), \ell_{g^{\prime}}\right)<\infty$, there is a fibre-preserving quasi-isometry $f_{\gamma}: \mathcal{H}_{\gamma}^{\text {Solv }} \rightarrow \mathcal{H}_{\gamma^{\prime}}^{\text {SOLV }}$ such that $\sup _{x \in \mathcal{H}_{\gamma}^{\text {Solv }}} d\left(f_{\gamma}(x), f(x)\right)<\infty$. Proposition 5.2 of [FM02a] then says that $f_{\gamma}$ coarsely respects the stable and unstable 
foliations. In particular, by restricting to a fibre of $\mathcal{H}_{\gamma}^{\text {Solv }}$ and using the definition of $\theta(f)$, we deduce that $\theta(f)(E(g))=E\left(g^{\prime}\right)$ as required.

We also need the following elementary connect-the-dots argument:

Lemma A.15. For every $K \geq 1$ and $A \geq 0$, there is a constant $B$ such that for every $(K, A)$-quasi-isometry $f: X_{L} \rightarrow X_{L}$ and every line $\ell \subseteq X_{L}$, there is a line $\ell^{\prime} \subseteq X_{L}$ with $d_{\text {Haus }}\left(f(\ell), \ell^{\prime}\right) \leq B$.

Proof. As $\ell$ is a line, there is a piecewise geodesic map $r: \mathbb{R} \rightarrow \mathcal{T}$ with image $\ell$. By reparametrising $\mathbb{R}$, we may assume that $r$ is 1-Lipschitz. We define a map $r^{\prime}: \mathbb{R} \rightarrow \mathcal{T}$ such that for all $n \in \mathbb{Z},\left.r^{\prime}\right|_{[n, n+1]}$ is a reparametrised Teichmüller geodesic segment in $X_{L}$ from $f(r(n))$ to $f(r(n+1))$. Thus the image of $r^{\prime}$ is a line $\ell^{\prime} \subseteq X_{L}$ with $d_{\text {Haus }}\left(f(\ell), \ell^{\prime}\right) \leq B$ for some suitable $B$.

Proof of Proposition A.13. Suppose $f: \mathcal{H}_{L} \rightarrow \mathcal{H}_{L}$ is a $(K, A)$-quasi-isometry such that $\theta(f)=\operatorname{id}_{S^{1}}$. Let $\hat{f}: X_{L} \rightarrow X_{L}$ be the induced quasi-isometry as in Proposition A.12, which can be taken to be a $\left(K^{\prime}, A^{\prime}\right)$-quasi-isometry for some $K^{\prime} \geq 1$ and $A^{\prime} \geq 0$ depending only on $K$ and $A$. Let $g \in L$ be a pseudoAnosov with coarse axis $\ell_{g} \subseteq X_{L}$ and axis $\gamma_{g}$. By Lemma A.15, there is a line $\ell_{g}^{\prime} \subseteq \mathcal{T}$ such that $d_{\text {Haus }}\left(\hat{f}\left(\ell_{g}\right), \ell_{g}^{\prime}\right) \leq B$ for some $B=\left(K^{\prime}, A^{\prime}\right)$. Since $\ell_{g}$ is a coarse axis and so has finite Hausdorff distance from the geodesic $\gamma_{g}$, Theorem $A .4$ says that $\mathcal{H}_{\ell_{g}}$ is $\delta$-hyperbolic for some $\delta$. Thus $f$ induces a quasiisometry $\mathcal{H}_{\ell_{g}} \rightarrow \mathcal{H}_{\ell_{g}^{\prime}}$, so that $\mathcal{H}_{\ell_{g}^{\prime}}$ is $\delta^{\prime}=\delta^{\prime}(K, A, \delta)$-hyperbolic. Theorem A.4 implies $\ell_{g}^{\prime}$ has finite Hausdorff distance from some Teichmüller geodesic $\gamma_{g}^{\prime}$. Since $f$ induces a fibre-preserving quasi-isometry $\mathcal{H}_{\gamma_{g}} \rightarrow \mathcal{H}_{\gamma_{g}^{\prime}}$, Proposition A.5 then says that $\gamma_{g}^{\prime}$ is the axis of a pseudo-Anosov $g^{\prime} \in \operatorname{MCG}(S)$. By Proposition A.11, we can assume that $g^{\prime} \in L$ and so $\ell_{g}^{\prime}$ is a coarse axis of $g^{\prime}$. Thus Proposition A.14 says that $E\left(g^{\prime}\right)=\theta(f)(E(g))=E(g)$ and so Lemma A.2 ensures that $g$ and $g^{\prime}$ share an axis. Thus $\gamma_{g}=\gamma_{g}^{\prime}$ and so $d_{\text {Haus }}\left(\ell_{g}, \widehat{f}\left(\ell_{g}\right)\right)<\infty$.

We now fix some conjugacy class $\mathcal{C}$ of pseudo-Anosovs in $L$ and equivariantly pick coarse axes $\left\{l_{g} \subseteq X_{L} \mid g \in \mathcal{C}\right\}$. Thus there is a $\delta$ such that $\mathcal{H}_{\ell_{g}}$ is $\delta$-hyperbolic for every $g \in \mathcal{C}$. Hence there is $\delta^{\prime}=\delta^{\prime}(\delta, K, A)$ and $B$ such that for all $g \in \mathcal{C}$, there is a line $\ell_{g}^{\prime}$ such that $d_{\text {Haus }}\left(\hat{f}\left(\ell_{g}\right), \ell_{g}^{\prime}\right) \leq B$ and $\mathcal{H}_{\ell_{g}^{\prime}}$ is $\delta^{\prime}$-hyperbolic. Theorem A.4 then says that there is some $C=C\left(\delta, \delta^{\prime}, L\right)$ such that for all $g \in \mathcal{C}$, both $\ell_{g}$ and $\ell_{g}^{\prime}$ have Hausdorff distance at most $C$ from a Teichmüller geodesic. By the argument in the previous paragraph and Lemma A.3, we deduce $\ell_{g}$ and $\ell_{g}^{\prime}$ have Hausdorff distance at most $C$ from the same Teichmüller geodesic, and so there is a $D \geq 0$ such that $d_{\text {Haus }}\left(\ell_{g}, \hat{f}\left(\ell_{g}\right)\right) \leq D$ for every $g \in \mathcal{C}$.

Let $g, h \in \mathcal{C}$ be two pseudo-Anosovs such that $\operatorname{Fix}(g) \cap \operatorname{Fix}(h)=\emptyset$. We fix some bounded $\Omega \subseteq \mathcal{T}$ such that $X_{L} \subseteq L \Omega$ and pick $r$ large enough so that $d\left(\omega, \ell_{g}\right), d\left(\omega, \ell_{h}\right) \leq r$ for all $\omega \in \Omega$. By Lemma A.3 there is a constant $R$ such that if $d\left(\sigma, \ell_{g}\right), d\left(\sigma, \ell_{h}\right) \leq K^{\prime} r+A^{\prime}+D$ for some $\sigma \in \mathcal{T}$, then $d(\sigma, \omega) \leq R$ for all $\omega \in \Omega$. Let $\sigma \in X_{L}$. Pick $k \in L$ and $\omega \in \Omega$ such that $\sigma=k \omega$. Thus $d\left(\sigma, k \ell_{g}\right), d\left(\sigma, k \ell_{h}\right) \leq r$. Notice that $k \ell_{g}=\ell_{k g k^{-1}}$ and $k \ell_{h}=\ell_{k h k^{-1}}$. 
Then $d\left(\hat{f}(\sigma), k \ell_{g}\right), d\left(\hat{f}(\sigma), k \ell_{h}\right) \leq K^{\prime} r+A^{\prime}+D$ so that $d(\hat{f}(\sigma), \sigma) \leq R$ for all $\sigma \in X_{L}$.

It follows from the definition of $\hat{f}$ that there is a constant $E=E(K, A, R)$ such that for every fibre $D_{\sigma} \subseteq \mathcal{H}_{L}, d_{\text {Haus }}\left(D_{\sigma}, f\left(D_{\sigma}\right)\right) \leq E$. Proposition 2.10 tells us that there exists $K^{\prime \prime} \geq 1$ and $A^{\prime \prime} \geq 0$ such that for every $\sigma \in X_{L}$, there is a $\left(K^{\prime \prime}, A^{\prime \prime}\right)$-quasi-isometry $f_{\sigma}: D_{\sigma} \rightarrow D_{\sigma}$ such that $\sup _{x \in D_{\sigma}} d\left(f_{\sigma}(x), f(x)\right) \leq E$. Since $\theta(f)=\operatorname{id}_{S^{1}}$, the induced boundary homeomorphism $\partial f_{\sigma}: \partial D_{\sigma} \rightarrow \partial D_{\sigma}$ is the identity. Hence by Theorem A.7, there is an $F=F\left(K^{\prime \prime}, A^{\prime \prime}\right)$ such that $\sup _{x \in D_{\sigma}} d\left(x, f_{\sigma}(x)\right) \leq F$. Thus $\sup _{x \in \mathcal{H}_{L}} d(x, f(x)) \leq E+F$. Notice that the constants $E, F$ depend only on the constants $K$ and $A$. (They also depend on the choice of conjugacy class $\mathcal{C}$, the elements $g, h \in \mathcal{C}$ and the choice of coarse axes, but these can be fixed as we vary $f$ over all $(K, A)$-quasi-isometries.)

Let $g \in L$ be a pseudo-Anosov with axis $\gamma$. Recall that $\operatorname{Isom}_{h}\left(\mathcal{H}_{\gamma}^{\text {Solv }}\right)$ is the subgroup of isometries of $\mathcal{H}_{\gamma}^{\text {SoLv }}$ that preserve each fibre. For ease of notation, let us denote $\operatorname{Isom}_{h}\left(\mathcal{H}_{\gamma}^{\mathrm{SoLv}}\right)$ by $\Pi_{g}$. Choosing some $\sigma \in \gamma, \Pi_{g}$ restricts to a group of isometries $\operatorname{Isom}\left(D_{\sigma}\right)$. Since $D_{\sigma}$ has finite Hausdorff distance from $D_{0}$, we have an induced homomorphism $\Pi_{g} \rightarrow \operatorname{QSym}\left(S^{1}\right)$, where $S^{1}$ is identified with the Gromov boundary of $D_{0}$. Any isometry of $D_{\sigma} \cong \mathbb{H}^{2}$ that extends to the identity map of $S^{1}$ must be the identity on $D_{\sigma}$. Thus $\Pi_{g} \rightarrow \operatorname{QSym}\left(S^{1}\right)$ is injective and so $\Pi_{g}$ can be regarded as a subgroup of $\operatorname{QSym}\left(S^{1}\right)$.

Let $\Pi:=\cap\left\{\Pi_{g} \mid g \in L\right.$ is a pseudo-Anosov $\}$. We recall that each $\Pi_{g}$ contains $\pi_{1}(S)$ as a finite index subgroup. Hence $\Pi$ can be identified with a subgroup of Isom $\left(\mathbb{H}^{2}\right)$ that contains $\pi_{1}(S)$ as a finite index subgroup. Let $\mathcal{O}$ denote the quotient orbifold $\Pi \backslash \mathbb{H}^{2}$ with orbifold fundamental group $\Pi$. Then there is a finite cover $q: S \rightarrow \mathcal{O}$ which induces the inclusion $q_{*}: \pi_{1}(S) \rightarrow \Pi$. As remarked in FM02a and Mos03a], the preceding theory generalises to the mapping class group and Teichmüller space of $\mathcal{O}$. Let $\mathcal{T}_{\mathcal{O}}$ denote the Teichmüller space of $\mathcal{O}$.

Lemma A.16. Recall the surface group extension $\Gamma_{L}$ can be naturally identified with a subgroup of $\operatorname{Aut}\left(\pi_{1}(S)\right)$. Then there is a unique map $\phi: \Gamma_{L} \rightarrow$ Aut(П) such that:

(1) $\left.\phi(\alpha)\right|_{\pi_{1}(S)}=\alpha$;

(2) there is a monomorphism $\bar{\phi}: L \hookrightarrow \operatorname{Out}(\Pi)$ such that the following diagram commutes

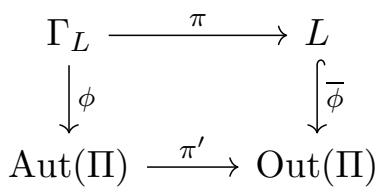

where $\pi$ and $\pi^{\prime}$ are quotient maps.

Proof. We first note that Lemma A.8 and Corollary A.10 imply that any automorphism of $\Gamma$ that restricts to an automorphism of $\pi_{1}(S)$ is determined by its restriction. Thus if $\phi$ exists, it is unique. Suppose $h \in \Gamma_{L}$ and $g \in L$ is a pseudo-Anosov with axis $\gamma$. Then $\pi(h) \gamma$ is the axis of the 
pseudo-Anosov $\pi(h) g \pi(h)^{-1}$. Thus $h$ restricts to a fibre-preserving isometry $\mathcal{H}_{\gamma}^{\text {SoLv }} \rightarrow \mathcal{H}_{\pi(h) \gamma}^{\text {Solv }}$, hence $h$ conjugates $\Pi_{g}$ to $\Pi_{\pi(h) g \pi(h)^{-1}}$. This holds for every pseudo-Anosov $g \in L$, so we deduce that $h$ defines an automorphism of $\Pi$ that we denote $\phi(h)$.

Suppose there exist elements $g, h \in \Gamma_{L}$ such that $\pi(g)=\pi(h)$. Then $g^{-1} h$ is an inner automorphism of $\pi_{1}(S)$, so clearly extends to an inner automorphism of $\Pi$. Thus $\pi^{\prime} \circ \phi(g)=\pi^{\prime} \circ \phi(h)$, hence $\phi$ induces a welldefined homomorphism $\bar{\phi}: L \rightarrow \operatorname{Out}(\Pi)$ such that $\pi^{\prime} \circ \phi=\bar{\phi} \circ \pi$.

Finally, we claim that $\bar{\phi}$ is injective. Suppose that $\pi(h) \in \operatorname{ker}(\bar{\phi})$. Then $\phi(h)$ is an inner automorphism of $\Pi$, so via the the Dehn-Nielsen-Baer theorem, corresponds to an orbifold homeomorphism $B$ of $\mathcal{O}$ that is isotopic to the identity. We can lift this isotopy to an isotopy of $S$ from the identity to a lift $A$ of $B$. Thus $A$ is a homeomorphism of $S$ that represents the trivial mapping class. Since $A$ is a lift of $B$, it follows that $A$ is a homeomorphism of $S$ that represents $h$, and so $h$ must be an inner automorphism of $\pi_{1}(S)$ via the the Dehn-Nielsen-Baer theorem.

Lemma A.16 has a topological interpretation. Via the Dehn-NielsenBaer theorem, the image of $\bar{\phi}$ can be naturally identified with a subgroup of $\operatorname{MCG}(\mathcal{O})$. It follows from Lemma A.16 and the Dehn-Nielsen-Baer theorem that for each $l \in L$, there are homeomorphisms $A$ of $S$ and $B$ of $\mathcal{O}$ representing $l$ and $\bar{\phi}(l)$ respectively, such that $A$ is a lift of $B$. In the terminology of Mos03a, Lemma A.16 says that $L \leq \operatorname{MCG}(S)$ descends to a subgroup of $\operatorname{MCG}(\mathcal{O})$. It is shown in Mos03a that $\mathcal{O}$ is the smallest orbifold such that $L$ descends.

We let $L^{\prime}:=\operatorname{im}(\bar{\phi}) \cong L$ and $\Gamma_{L^{\prime}}:=\pi^{\prime-1}\left(L^{\prime}\right)$. As the inclusion $\pi_{1}(S) \rightarrow \Pi$ has finite index image, we see that $\Gamma_{L}$ maps to a finite index subgroup of $\Gamma_{L^{\prime}}$, which we identify with $\Gamma_{L}$. We observe that there is an embedding $\mathcal{T}_{\mathcal{O}} \rightarrow \mathcal{T}_{S}=\mathcal{T}$. This is because a hyperbolic structure on $\mathcal{O}$ can be lifted to a hyperbolic structure on $S$. (Alternatively, a discrete faithful representation $\Pi \rightarrow \operatorname{PSL}(2, \mathbb{R})$ restricts to a discrete faithful representation $\pi_{1}(S) \rightarrow \operatorname{PSL}(2, \mathbb{R})$.) Teichmüller's theorem implies that this in fact an isometric embedding, since Teichmüller geodesics in $\mathcal{T}_{\mathcal{O}}$ map to Teichmüller geodesics in $\mathcal{T}_{S}$. We thus identify $\mathcal{T}_{\mathcal{O}}$ with a subset of $\mathcal{T}$. Since $L^{\prime} \leq \operatorname{Out}(\Pi) \cong \operatorname{MCG}(\mathcal{O})$, we can always ensure that $X_{L}$, as defined above, is a subset of $\mathcal{T}_{\mathcal{O}}$. The canonical hyperbolic plane bundle $\mathcal{H}_{\mathcal{O}} \rightarrow \mathcal{T}_{\mathcal{O}}$ associated to $\mathcal{O}$ is thus a pullback of the canonical hyperbolic plane bundle $\mathcal{H} \rightarrow \mathcal{T}$ associated to $S$.

Let $M=\operatorname{Comm}_{\mathrm{MCG}(\mathcal{O})}\left(L^{\prime}\right)$ and let $\Gamma_{M}$ be the corresponding subgroup of $\operatorname{Aut}(\Pi) \cong \widetilde{\mathrm{MCG}}(\mathcal{O})$. For every $g \in M, d_{\text {Haus }}\left(L^{\prime}, g L^{\prime}\right)<\infty$ by Proposition 3.12, from which it follows that $d_{\text {Haus }}\left(\Gamma_{L^{\prime}}, h \Gamma_{L^{\prime}}\right)<\infty$ for all $h \in \Gamma_{M}$. Since $\Gamma_{L}$ is a finite index subgroup of $\Gamma_{L^{\prime}}$, we see that $d_{\text {Haus }}\left(\Gamma_{L}, h \Gamma_{L}\right)<\infty$ for all $h \in \Gamma_{M}$. Thus for each $h \in \Gamma_{M}$, left multiplication by $h$ followed by closest point projection defines a quasi-isometry $\Gamma_{L} \rightarrow \Gamma_{L}$. This gives a homomorphism $\Psi: \Gamma_{M} \rightarrow \operatorname{QIsom}\left(\Gamma_{L}\right)$. We classify all quasi-isometries of $\Gamma_{L}$ as follows:

Theorem A.17. The map $\Psi: \Gamma_{M} \rightarrow \mathrm{QIsom}\left(\Gamma_{L}\right)$ is an isomorphism. 
For injectivity we note that Lemma A.8 ensures two distinct elements $g, h \in \Gamma_{M} \leq \widehat{\operatorname{MCG}}(\mathcal{O}) \cong \operatorname{Aut}(\Pi)$ induce different elements of $\operatorname{QSym}\left(S^{1}\right)$, hence Proposition A.13 ensures that $\Psi(g) \neq \Psi(h)$. Surjectivity follows immediately from the following lemma.

Lemma A.18. Let $K \geq 1$ and $A \geq 0$. Then there is a constant $C=$ $C(K, A)$ such that whenever $f$ is a $(K, A)$-quasi-isometry $f: \Gamma_{L} \rightarrow \Gamma_{L}$, there exists a $\psi_{f} \in \Gamma_{M}$ such that for all $x \in \Gamma_{L}$,

$$
d_{\widetilde{\mathrm{MCG}}(\mathcal{O})}\left(\psi_{f} x, f(x)\right) \leq C .
$$

Proof. The argument is similar to the proof of Proposition A.13, It is enough to show the following: there is a constant $C=C(K, A)$ such that whenever $f$ is a $(K, A)$-quasi-isometry $f: \mathcal{H}_{L} \rightarrow \mathcal{H}_{L}$, there exists a $\psi_{f} \in \Gamma_{M}$ such that for all $x \in \Gamma_{L}$,

$$
d_{\mathcal{H}_{\mathcal{O}}}\left(\psi_{f} x, f(x)\right) \leq C .
$$

This is because $\Gamma_{L}$ acts properly discontinuously and cocompactly on $\mathcal{H}_{L}$, and the orbit map $\widehat{\operatorname{MCG}}(\mathcal{O}) \rightarrow \mathcal{H}_{\mathcal{O}}$ is a coarse embedding.

Suppose $f: \mathcal{H}_{L} \rightarrow \mathcal{H}_{L}$ is a $(K, A)$-quasi-isometry. Proposition A.12 ensures there are constants $K^{\prime}, A^{\prime}$ and $B$ such that there is a $\left(K^{\prime}, A^{\prime}\right)$-quasiisometry $\hat{f}: X_{L} \rightarrow X_{L}$ such that $d_{\text {Haus }}\left(f\left(D_{\sigma}\right), D_{\hat{f}(\sigma)}\right) \leq B$ for all $\sigma \in X_{L}$. Let $g \in L$ be a pseudo-Anosov with coarse axis $\ell_{g} \subseteq X_{L}$ and axis $\gamma_{g} \subseteq \mathcal{T}_{\mathcal{O}} \subseteq$ $\mathcal{T}_{S}$. By Lemma A.15, there is a line $\ell_{g}^{\prime} \subseteq X_{L}$ such that $d_{\text {Haus }}\left(\hat{f}\left(\ell_{g}\right), \ell_{g}^{\prime}\right) \leq C$ for some $C=\left(K^{\prime}, A^{\prime}\right)$. Since $\ell_{g}$ is a coarse axis, $\mathcal{H}_{\ell_{g}}$ is $\delta$-hyperbolic for some $\delta$. Thus $f$ induces a quasi-isometry $f_{g}: \mathcal{H}_{\ell_{g}} \rightarrow \mathcal{H}_{\ell_{g}^{\prime}}$, so that $\mathcal{H}_{\ell_{g}^{\prime}}$ is $\delta^{\prime}$ hyperbolic and therefore has finite Hausdorff distance from some Teichmüller geodesic $\gamma_{g}^{\prime}$.

By Propositions A.5 and A.11, we deduce that $\gamma_{g}^{\prime}$ is the axis of a pseudoAnosov $g^{\prime} \in L$ and that there is a fibre-preserving isometry $\mathcal{H}_{\ell_{g}} \rightarrow \mathcal{H}_{\ell_{g}^{\prime}}$. This isometry conjugates $\operatorname{Isom}_{h}\left(\mathcal{H}_{\ell_{g}}\right)$ to $\operatorname{Isom}_{h}\left(\mathcal{H}_{\ell_{g}^{\prime}}\right)$, so that $\theta(f)$ conjugates $\Pi_{g}$ to $\Pi_{g^{\prime}}$ in $\operatorname{QSym}\left(S^{1}\right)$. Since this holds for each pseudo-Anosov $g \in L, \theta(f)$ conjugates $\Pi$ in QSym $\left(S^{1}\right)$. Thus Corollary A.10 and Proposition $\mathrm{A} .13$ ensures there is an element $\psi_{f} \in \widehat{\operatorname{MCG}}(\mathcal{O}) \cong \operatorname{Aut}(\Pi)$ such that $\sup _{x \in X_{L}} d_{\mathcal{H}_{\mathcal{O}}}\left(f(x), \psi_{f} \cdot x\right)<\infty$.

To complete the proof, we need only show that $\sup _{x \in X_{L}} d_{\mathcal{H}_{\mathcal{O}}}\left(f(x), \psi_{f} \cdot x\right)$ can be bounded by a constant that depends only on $K$ and $A$ and not the quasi-isometry $f$. We argue similarly to the proof of Proposition A.13. We fix a conjugacy class $\mathcal{C}$ of pseudo-Anosovs in $L$ and choose coarse axes $\left\{\ell_{g} \mid g \in \mathcal{C}\right\}$ equivariantly. Thus there is a $\delta$ such that $\mathcal{H}_{\ell_{g}}$ is $\delta$-hyperbolic for every $g \in \mathcal{C}$. There is therefore a $\delta^{\prime}$ such that $\mathcal{H}_{\ell_{g}^{\prime}}$ is $\delta^{\prime}$-hyperbolic for every $g \in \mathcal{C}$.

Let $\phi_{f}:=\pi^{\prime}\left(\sigma_{f}\right) \in L^{\prime}$, where $\pi^{\prime}: \operatorname{Aut}(\Pi) \rightarrow \operatorname{Out}(\Pi) \cong \operatorname{MCG}(\mathcal{O})$ is the quotient map. As $\phi_{f}$ acts by isometries on $\mathcal{T}_{\mathcal{O}}, \phi_{f} \ell_{g}$ is a hyperbolic line in $\mathcal{T}_{\mathcal{O}}$ such that $\mathcal{H}_{\phi_{f} \ell_{g}}=\psi_{f} \mathcal{H}_{\ell_{g}}$ is $\delta$-hyperbolic. Since $\sup _{x \in X_{L}} d_{\mathcal{H}_{\mathcal{O}}}\left(f(x), \psi_{f} \cdot x\right)<$ $\infty$, it follows that $d_{\text {Haus }} d_{\mathcal{T}_{\mathcal{O}}}\left(\hat{f}\left(\ell_{g}\right), \phi_{f}\left(\ell_{g}\right)\right)<\infty$. Using Theorem A.4, there is a constant $D=D\left(\delta, \delta^{\prime}\right)$ such that $d_{\text {Haus }}\left(\hat{f}\left(\ell_{g}\right), \phi_{f}\left(\ell_{g}\right)\right) \leq D$ for each $g \in \mathcal{C}$. Furthermore, there is a constant $E$ such that every point $x \in X_{L}$ satisfies $x \in N_{E}\left(\ell_{g}\right)$ for some $g \in \mathcal{C}$. Thus $\phi_{f} \cdot X_{L} \subseteq N_{D+E}\left(X_{L}\right)$ and so 
$\psi_{f} \cdot \mathcal{H}_{L} \subseteq N_{D+E}\left(\mathcal{H}_{L}\right)$. As usual, the constants $D$ and $E$ don't depend on $f$, only the constants $K$ and $A$

To conclude, we define a closest point projection map $u: N_{D+E}\left(\mathcal{H}_{L}\right) \rightarrow$ $\mathcal{H}_{L}$ whose quasi-isometry constants depend only on $D+E$. Thus there exist constants $K^{\prime \prime}$ and $A^{\prime \prime}$ such that for every $(K, A)$-quasi-isometry $f: \mathcal{H}_{L} \rightarrow$ $\mathcal{H}_{L}$, the composition $\bar{f} \circ u \circ \psi_{f}: \mathcal{H}_{L} \rightarrow \mathcal{H}_{L}$ is a $\left(K^{\prime \prime}, A^{\prime \prime}\right)$-quasi-isometry, where $\bar{f}$ is a coarse inverse to $f$. Thus Proposition A.13 ensures that there is a constant $F$ such that $\sup _{\mathcal{H}_{L}} d\left(\bar{f} \circ u \circ \psi_{f}(x), x\right) \leq F$ for every $(K, A)$ quasi-isometry $f: \mathcal{H}_{L} \rightarrow \mathcal{H}_{L}$. The result immediately follows.

We deduce Theorem A.19 from Lemma A.18 using an argument similar to that found in Sch95.

Theorem A.19. Suppose $L$ is an irreducible subgroup of $\operatorname{MCG}(S)$ that is of type $F_{3}$ and has infinitely many ends. Let $\Gamma_{L}$ be the associated surface group extension. If $G$ is any finitely generated group quasi-isometric to $\Gamma_{L}$, then there is a finite normal subgroup $N \triangleleft G$ such that $G / N$ is abstractly commensurable to $\Gamma_{L}$, i.e. $\Gamma_{L}$ and $G / N$ have isomorphic finite index subgroups.

Proof. We fix an irreducible, infinite-ended subgroup $L \leq \operatorname{MCG}(S)$ of type $F_{3}$. Suppose $f: G \rightarrow \Gamma_{L}$ is a quasi-isometry, where $G$ is any finitely generated group. Let $\bar{f}$ be a coarse inverse to $f$ and for each $g \in G$, let $L_{g}: G \rightarrow G$ be left multiplication by $g$. Then $f_{g}:=f \circ L_{g} \circ \bar{f}$ is a quasiisometry of $\Gamma_{L}$. Since $L_{g}$ is an isometry of $\Gamma_{L},\left\{f_{g} \mid g \in G\right\}$ is a uniform set of quasi-isometries, i.e. there exists $K \geq 1$ and $A \geq 0$ such that each $f_{g}$ is a $(K, A)$-quasi-isometry. We may also assume that $f$ and $\bar{f}$ are $(K, A)$-quasiisometries. Thus Lemma A.18 ensures there is a $C \geq 0$ such that for every $g \in G$ there exists an $\psi_{g} \in \Gamma_{M}$ with

$$
d\left(\psi_{g} \cdot x, f_{g}(x)\right) \leq C
$$

for all $x \in \Gamma_{L}$. We claim that the map $\Psi: G \rightarrow \Gamma_{M}$ given by $g \mapsto \psi_{g}$ has finite kernel and image commensurable to $\Gamma_{L} \leq \Gamma_{M}$. This map is welldefined and a homomorphism.

To shown $\operatorname{ker}(\Psi)$ is finite, we pick $x_{0} \in G$ such that $d\left(f\left(x_{0}\right), e\right) \leq A$ and suppose that $g \in \operatorname{ker}(\Psi)$. Then $d\left(e, f_{g}(e)\right) \leq C$. Thus

$$
\begin{aligned}
\frac{1}{K} d\left(x_{0}, g x_{0}\right)-A & \leq d\left(f\left(x_{0}\right), f\left(g x_{0}\right)\right) \\
& \leq d\left(f\left(x_{0}\right), e\right)+d\left(e, f_{g}(e)\right)+d\left(f_{g}(e), f\left(g x_{0}\right)\right) \\
& \leq K d\left(\bar{f}(e), x_{0}\right)+2 A+C .
\end{aligned}
$$

There are only finitely many such $g \in G$, thus $\operatorname{ker}(\Psi)$ is finite.

We now show $\operatorname{im}(\Psi)$ has finite Hausdorff distance from $\Gamma_{L}$. For each $x \in \Gamma_{L}$, there exists some $k_{x} \in G$ with $d\left(f\left(k_{x}\right), x\right) \leq A$. Let $g_{x}:=k_{x} \bar{f}(e)^{-1}$. Then $f_{g_{x}}(e)=f\left(g_{x} \bar{f}(e)\right)=f\left(k_{x}\right)$ and so

$$
d\left(\psi_{g_{x}}, x\right) \leq d\left(\psi_{g_{x}} \cdot e, f_{g_{x}}(e)\right)+d\left(f\left(k_{x}\right), x\right) \leq C+A,
$$

thus $\Gamma_{L} \subseteq N_{A+C}(\operatorname{im} \Psi)$. Conversely for each $\psi_{g} \in \operatorname{im} \Psi, d\left(\psi_{g}, f_{g}(e)\right) \leq C$, so that $\operatorname{im} \Psi \subseteq N_{C}\left(\Gamma_{L}\right)$. 


\section{Appendix B. Groups QuASI-ISOMETRIC to PRODUCTS}

In this appendix we demonstrate further instances in which Question 1.2 is true, even if Theorem 1.4 doesn't necessarily apply. Theorem B.5 is not a new result in and of itself, rather it is a combination of existing results - namely [KKL98] and MSW03] - formulated in the language of almost normal subgroups and quotient spaces. Theorem B.5 can be used to deduce Proposition 1.3 .

The following theorem says that under some fairly general coarse nonpositive curvature assumptions, quasi-isometries preserve direct products. We refer to [KKL98] for a definition of coarse type I and II.

Theorem B.1 ([KKL98, Theorem B]). Suppose $X=Z \times \Pi_{i=1}^{k} X_{i}$ is a geodesic metric space such that the asymptotic cone of $Z$ is homeomorphic to $\mathbb{R}^{n}$ and each $X_{i}$ is either of coarse type $I$ or $I I$. Let $p_{i}: X \rightarrow X_{i}$ be the projection map. Then for every $K \geq 1$ and $A \geq 0$, there exist constants $K^{\prime} \geq 1$ and $A^{\prime}, D \geq 0$ such that the following holds:

Whenever $f: X \rightarrow X$ is a $(K, A)$-quasi-isometry, there is a $\sigma_{f} \in \operatorname{Sym}(k)$ so that for each $i$, there exists a $\left(K^{\prime}, A^{\prime}\right)$-quasi-isometry $f_{i}$ such that the following diagram commutes up to error at most $D$.

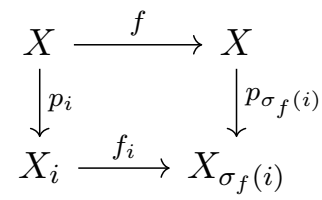

We recall the definition of a quasi-action:

Definition B.2. Let $G$ be a group and $X$ be a metric space. A quasi-action of $G$ on $X$ associates to each $g \in G$ a quasi-isometry $f_{g}: X \rightarrow X$ such that:

(1) there are constants $K \geq 1$ and $A \geq 0$ such that each $f_{g}$ is a $(K, A)$ quasi-isometry;

(2) for all $g, h \in G$ and $x \in X, d\left(f_{h}\left(f_{g}(x)\right), f_{h g}(x)\right) \leq A$;

(3) for all $x \in X, d\left(f_{e}(x), x\right) \leq A$.

For $(K, A)$ as above, we say that $\left\{f_{g}\right\}_{g \in G}$ is a $(K, A)$-quasi-action.

Lemma B.3. Suppose a finitely generated group $G$ quasi-acts on a metric space $X=Z \times \prod_{i=1}^{k} X_{i}$ as in Theorem B.1. Then for each $i$, there is a finite index subgroup $G_{0} \leq G$ such that for $1 \leq i \leq k, G_{0}$ admits a quasi-action on $X_{i}$.

Proof. Let $\left\{f_{g}\right\}_{g} \in G$ be the set of quasi-isometries associated to the quasiaction of $G$ on $X$. We apply Theorem B.1 to each $f_{g}$. The map $f_{g} \mapsto \sigma_{f_{g}}$ defines a homomorphism $G \rightarrow \operatorname{Sym}(k)$ whose kernel we denote $G_{0}$. Then the set $\left\{\left(f_{g}\right)_{i}: X_{i} \rightarrow X_{i} \mid g \in G_{0}\right\}$ defines a quasi-action of $G_{0}$ on $X_{i}$. Note that $G=G_{0}$ if none of the $X_{i}$ are quasi-isometric to one another.

Given two quasi-actions $\left\{f_{g}\right\}_{g \in G}$ and $\left\{k_{g}\right\}_{g \in G}$ on metric spaces $X$ and $Y$, a quasi-conjugacy is a quasi-isometry $r: X \rightarrow Y$ such that there exists a constant $A \geq 0$ with $d_{Y}\left(\left(k_{g} \circ r\right)(x),\left(r \circ f_{g}\right)(x)\right) \leq A$ for all $x \in X$ and $g \in G$. A bushy tree is one in which every point of $T$ has uniformly bounded distance from a vertex with at least three unbounded complementary components. We can now state the following theorem of Mosher-Sageev-Whyte: 
Theorem B.4 ([MSW03]). Let $G$ be a group admitting a cobounded quasiaction on a bounded valence bushy tree $T$. Then $G$ acts isometrically on a bounded valence, bushy tree $T^{\prime}$ and there is a quasi-conjugacy $r: T^{\prime} \rightarrow T$.

Proposition 1.3 can be deduced as a special case of the following:

Theorem B.5. Let $G$ be a finitely generated group quasi-isometric to a metric space $X=Z \times \Pi_{i=1}^{k} X_{i}$ as in Theorem B.1. Suppose that $X_{1}$ is a bounded valence bushy tree. Then there exists a finite index subgroup $G_{0} \leq G$ that contains an almost normal subgroup $H$ quasi-isometric to $Z \times \Pi_{i=2}^{k} X_{i}$, such that the quotient space $G_{0} / H$ is quasi-isometric to a finite valence bushy tree.

Proof. Suppose $f: G \rightarrow X$ is a quasi-isometry. Conjugating the left action of $G$ on itself by $f$ defines a quasi-action of $G$ on $X$. By Lemma B.3, we deduce that a subgroup $G_{0} \leq G$ of index at most $k$ ! admits a quasi-action on $X_{1}$. By Theorem B.4, $G_{0}$ acts on isometrically on a tree $T$ and this action is quasi-conjugate to the quasi-action of $G_{0}$ on $X_{1}$. Let $H$ be a stabiliser of an edge of $T$. Since $T$ is a bounded valence tree, every conjugate of $H$ is commensurable to $H$ and so $H \backsim G_{0}$. The quotient space $G_{0} / H$ is quasi-isometric to $T$. Using the quasi-conjugacy $T \rightarrow X_{1}$ and Theorem B.1, we deduce $f(H)$ has finite Hausdorff distance from $p_{1}^{-1}(x)$ for some $x \in X$, demonstrating that $H$ is quasi-isometric to $Z \times \Pi_{i=2}^{k} X_{i}$.

\section{REFERENCES}

[AAS07] James W. Anderson, Javier Aramayona, and Kenneth J. Shackleton, Free subgroups of surface mapping class groups, Conform. Geom. Dyn. 11 (2007), 44-55. MR 2295997

[BA56] A. Beurling and L. Ahlfors, The boundary correspondence under quasiconformal mappings, Acta Math. 96 (1956), 125-142. MR 0086869

[Bel93] V. V. Belyaev, Inert subgroups in infinite simple groups, Sibirsk. Mat. Zh. 34 (1993), no. 4, 17-23, i, vii. MR 1248784

[BF91] Mladen Bestvina and Mark Feighn, Bounding the complexity of simplicial group actions on trees, Invent. Math. 103 (1991), no. 3, 449-469. MR 1091614

[BM97] Marc Burger and Shahar Mozes, Finitely presented simple groups and products of trees, C. R. Acad. Sci. Paris Sér. I Math. 324 (1997), no. 7, 747-752. MR 1446574

[Bow02] Brian H. Bowditch, Splittings of finitely generated groups over two-ended subgroups, Trans. Amer. Math. Soc. 354 (2002), no. 3, 1049-1078. MR 1867372

[Bow13] B. H. Bowditch, Stacks of hyperbolic spaces and ends of 3-manifolds, Geometry and topology down under, Contemp. Math., vol. 597, Amer. Math. Soc., Providence, RI, 2013, pp. 65-138. MR 3186670

[Bro87] Kenneth S. Brown, Finiteness properties of groups, J. Pure Appl. Algebra 44 (1987), no. 1-3, 45-75. MR 885095

[CM14] Gregory R. Conner and Michael L. Mihalik, Commensurated subgroups, semistability and simple connectivity at infinity, Algebr. Geom. Topol. 14 (2014), no. 6, 3509-3532. MR 3302969

[DK18] Cornelia Druţu and Michael Kapovich, Geometric group theory, American Mathematical Society Colloquium Publications, vol. 63, American Mathematical Society, Providence, RI, 2018, With an appendix by Bogdan Nica. MR 3753580

[Dun85] M. J. Dunwoody, The accessibility of finitely presented groups, Invent. Math. 81 (1985), no. 3, 449-457. MR 807066 
[EFW12] Alex Eskin, David Fisher, and Kevin Whyte, Coarse differentiation of quasiisometries I: Spaces not quasi-isometric to Cayley graphs, Ann. of Math. (2) 176 (2012), no. 1, 221-260. MR 2925383

[FLS15] Roberto Frigerio, Jean-François Lafont, and Alessandro Sisto, Rigidity of high dimensional graph manifolds, Astérisque (2015), no. 372, xxi+177. MR 3444648

[FM98] Benson Farb and Lee Mosher, A rigidity theorem for the solvable BaumslagSolitar groups, Invent. Math. 131 (1998), no. 2, 419-451, With an appendix by Daryl Cooper. MR 1608595

[FM99] Q Quasi-isometric rigidity for the solvable Baumslag-Solitar groups. II, Invent. Math. 137 (1999), no. 3, 613-649. MR 1709862

[FM00] - On the asymptotic geometry of abelian-by-cyclic groups, Acta Math. 184 (2000), no. 2, 145-202. MR 1768110

[FM02a] B. Farb and L. Mosher, The geometry of surface-by-free groups, Geom. Funct. Anal. 12 (2002), no. 5, 915-963. MR 1937831

[FM02b] Benson Farb and Lee Mosher, Convex cocompact subgroups of mapping class groups, Geom. Topol. 6 (2002), 91-152. MR 1914566

[FM12] Benson Farb and Dan Margalit, A primer on mapping class groups, Princeton Mathematical Series, vol. 49, Princeton University Press, Princeton, NJ, 2012. MR 2850125

[FS96] Benson Farb and Richard Schwartz, The large-scale geometry of Hilbert modular groups, J. Differential Geom. 44 (1996), no. 3, 435-478. MR 1431001

[Geo08] Ross Geoghegan, Topological methods in group theory, Graduate Texts in Mathematics, vol. 243, Springer, New York, 2008. MR 2365352

[Hop44] Heinz Hopf, Enden offener Räume und unendliche diskontinuierliche Gruppen, Comment. Math. Helv. 16 (1944), 81-100. MR 0010267

[HP18] Jingyin Huang and Tomasz Prytuła, Commensurators of abelian subgroups in $C A T(0)$ groups, arXiv preprint arXiv:1812.09035 (2018).

[Iva92] Nikolai V. Ivanov, Subgroups of Teichmüller modular groups, Translations of Mathematical Monographs, vol. 115, American Mathematical Society, Providence, RI, 1992, Translated from the Russian by E. J. F. Primrose and revised by the author. MR 1195787

[KK05] Michael Kapovich and Bruce Kleiner, Coarse Alexander duality and duality groups, J. Differential Geom. 69 (2005), no. 2, 279-352. MR 2168506

[KKL98] Michael Kapovich, Bruce Kleiner, and Bernhard Leeb, Quasi-isometries and the de Rham decomposition, Topology 37 (1998), no. 6, 1193-1211. MR 1632904

[KM08] Bernhard Krön and Rögnvaldur G. Möller, Analogues of Cayley graphs for topological groups, Math. Z. 258 (2008), no. 3, 637-675. MR 2369049

[Kob12] Thomas Koberda, Right-angled Artin groups and a generalized isomorphism problem for finitely generated subgroups of mapping class groups, Geom. Funct. Anal. 22 (2012), no. 6, 1541-1590. MR 3000498

[KR89] P. H. Kropholler and M. A. Roller, Relative ends and duality groups, J. Pure Appl. Algebra 61 (1989), no. 2, 197-210. MR 1025923

[Kro06] P. H. Kropholler, A generalization of the Lyndon-Hochschild-Serre spectral sequence with applications to group cohomology and decompositions of groups, J. Group Theory 9 (2006), no. 1, 1-25. MR 2195835

[LM19] I. Leary and A. Minasyan, Commensurating HNN-extensions: non-positive curvature and biautomaticity, To appear, 2019.

[Mar18] Alexander J. Margolis, Quasi-isometry invariance of group splittings over coarse Poincaré duality groups, Proc. Lond. Math. Soc. (3) 116 (2018), no. 6, 14061456. MR 3816385

[McC94] John D McCarthy, Normalizers and centralizers of pseudo-anosov mapping classes, preprint), June 8 (1994).

[Mos03a] Lee Mosher, Fiber respecting quasi-isometries of surface group extensions, arXiv preprint math/0308067 (2003).

[Mos03b] _ Stable Teichmüller quasigeodesics and ending laminations, Geom. Topol. 7 (2003), 33-90. MR 1988281 
[Mos09] Homology and dynamics in quasi-isometric rigidity of once-punctured mapping class groups, Geometric and cohomological methods in group theory, London Math. Soc. Lecture Note Ser., vol. 358, Cambridge Univ. Press, Cambridge, 2009, pp. 225-255. MR 2605178

[MSW03] Lee Mosher, Michah Sageev, and Kevin Whyte, Quasi-actions on trees. I. Bounded valence, Ann. of Math. (2) 158 (2003), no. 1, 115-164. MR 1998479

[MSW11] _ Quasi-actions on trees II: Finite depth Bass-Serre trees, Mem. Amer. Math. Soc. 214 (2011), no. 1008, vi+105. MR 2867450

[Pap05] Panos Papasoglu, Quasi-isometry invariance of group splittings, Ann. of Math. (2) 161 (2005), no. 2, 759-830. MR 2153400

[Pap07] _ Group splittings and asymptotic topology, J. Reine Angew. Math. 602 (2007), 1-16. MR 2300450

[Pau96] Frédéric Paulin, Un groupe hyperbolique est déterminé par son bord, J. London Math. Soc. (2) 54 (1996), no. 1, 50-74. MR 1395067

[PW02] Panos Papasoglu and Kevin Whyte, Quasi-isometries between groups with infinitely many ends, Comment. Math. Helv. 77 (2002), no. 1, 133-144. MR 1898396

[Roe93] John Roe, Coarse cohomology and index theory on complete Riemannian manifolds, Mem. Amer. Math. Soc. 104 (1993), no. 497, x+90. MR 1147350

[Roe03] Lectures on coarse geometry, University Lecture Series, vol. 31, American Mathematical Society, Providence, RI, 2003. MR 2007488

[Sch95] Richard Evan Schwartz, The quasi-isometry classification of rank one lattices, Inst. Hautes Études Sci. Publ. Math. (1995), no. 82, 133-168 (1996). MR 1383215

[Sco78] Peter Scott, Ends of pairs of groups, J. Pure Appl. Algebra 11 (1977/78), no. 13, 179-198. MR 487104

[SW13] Yehuda Shalom and George A. Willis, Commensurated subgroups of arithmetic groups, totally disconnected groups and adelic rigidity, Geom. Funct. Anal. 23 (2013), no. 5, 1631-1683. MR 3102914

[Vav13] Diane M. Vavrichek, The quasi-isometry invariance of commensurizer subgroups, Groups Geom. Dyn. 7 (2013), no. 1, 205-261. MR 3019080

[Why01] K. Whyte, The large scale geometry of the higher Baumslag-Solitar groups, Geom. Funct. Anal. 11 (2001), no. 6, 1327-1343. MR 1878322

[Why10] Kevin Whyte, Coarse bundles, arXiv preprint arXiv:1006.3347 (2010).

Alexander J. Margolis, Mathematics Department, Technion - Israel Institute of TeChNology, Haifa, 32000, IsRael

E-mail address: amargolis@campus.technion.ac.il 